\title{
A SURVEY OF REFLECTOMETRY TECHNIQUES WITH APPLICATIONS TO TFTR
}

\author{
Ivan Collazo, John Wilgen* \\ Greg Hanson*, Tim Bigelow* \\ Norton Bretz, $\uparrow$ W. M. Stacey, C. E. Thomas* \\ Fusion Research Center and \\ Nuclear Engineering Program \\ Georgia Institute of Technology \\ Atlanta, GA 30332 USA \\ December, 1993
}

\section{*Oak Ridge National Laboratory \\ †Princeton Plasma Physics Laboratory}

\section{DISCLAIMER}

This report was prepared as an account of work sponsored by an agency of the United States Government. Neither the United States Government nor any agency thereof, nor any of their employees, makes any warranty, express or implied, or assumes any legal liability or responsibility for the accuracy, completeness, or usefulness of any information, apparatus, product, or process disclosed, or represents that its use would not infringe privately owned rights. Reference herein to any specific commercial product, process, or service by trade name, trademark, manufacturer, or otherwise does not necessarily constitute or imply its endorsement, recom. mendation, or favoring by the United States Government or any agency thereof. The views and opinions of authors expressed herein do not necessarily state or reflect those of the United States Government or any agency thereof. 


\section{Summary}

This report presents a review of reflectometry with particular attention to eXtraordinary mode (X-mode) reflectometry using the novel technique of dual frequency differential phase. The advantage of using an X-mode wave is that it can probe the edge of the plasma with much higher resolution and using a much smaller frequency range than with the Ordinary mode (O-Mode). The general problem with previous full phase reflectometry techniques is that of keeping track of the phase (on the order of 1000 fringes) as the frequency is swept over the band. The dual frequency phase difference technique has the advantage that since it is keeping track of the phase difference of two frequencies with a constant frequency separation, the fringe counting is on the order of only 3 to 5 fringes. This fringe count, combined with the high resolution of the $\mathrm{X}$-mode wave and the small plasma access requirements of reflectometry, make X-mode reflectometry a very attractive diagnostic for today's experiments and future fusion devices. 


\section{Table of Contents}

Summary $\quad$ ii

Table of Contents $\quad$ iii

List of Figures vii

List of Tables $\quad$ x

I. Introduction 1

1.1 Fusion ........................ 1

1.2 Reflectometry ................... 6

1.3 Fusion Experiments . . . . . . . . . . . . . . 6

1.4 Tokamak Fusion Test Reactor . . . . . . . . . . . . . . 11

II. Reflectometry 14

2.1 History of Reflectometry . . . . . . . . . . . . . . 14

2.1.1 The beginings ................... 14

2.1.2 First experiments in Russia . . . . . . . . . . . 15

2.1.3 First experiment in USA ................. 15

2.2 Propagation of Electromagnetic Waves in Plasmas . . . . . . . 16

2.2 .1 Maxwell equations . . . . . . . . . . . . . 16

2.2 .2 The dielectric tensor . . . . . . . . . . . . 18

2.2.3 Properties of the $\mathrm{O}$ - and X-modes ........... 25

The Ordinary mode . . . . . . . . . . . . 25

The eXtraordinary mode .............. . 26 
2.2.4 Advantages and disadvantages of each mode . . . . . . 28

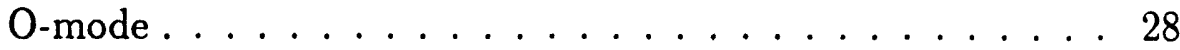

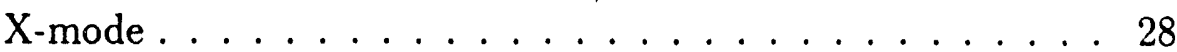

2.2.5 Solution to the wave equation in a plasma . . . . . . . 30

Geometrical optics solution . . . . . . . . . . . . 30

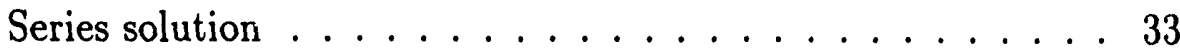

Solution at the reflection layer . . . . . . . . . . 34

One dimensional full wave analysis. . . . . . . . . . . 35

2.3 Reflectometry Techniques . . . . . . . . . . . . . . . 42

2.3.1 Fixed frequency . . . . . . . . . . . . . . 42

2.3.2 Narrow band swept frequency . . . . . . . . . . . 43

2.3.3 Broadband swept frequency . . . . . . . . . . . . . 43

2.3.4 AM technique ................. 45

2.3.5 Dual frequency phase difference technique . . . . . . . 48

2.3.6 Pulse radar technique . . . . . . . . . . . . 48

2.3.7 Other techniques ................. 49

Measurement of magnetic fluctuations . . . . . . . . . 49

Small scale edge fluctuations . . . . . . . . . . . . 49

2.4 Measuring Density Fluctuations . . . . . . . . . . . . . 50

2.4 .1 Using the $\mathrm{O}$-mode . . . . . . . . . . . . 52

2.4 .2 Using the X-mode . . . . . . . . . . . . 53

2.5 Measuring the Density Profile . . . . . . . . . . . . 53

2.5.1 Profile reconstruction using the $\mathrm{O}$-mode ......... 53

2.5.2 Profile reconstruction using the X-mode . . . . . . . . . 57

Full phase technique ................. 57

Phase difference technique ............ 58

2.6 Complications and Error Sources . . . . . . . . . . . . . 60 
2.6.1 Electron Cyclotron Resonance absorption . . . . . . . . 6 60

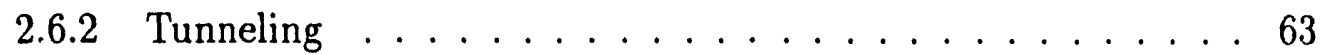

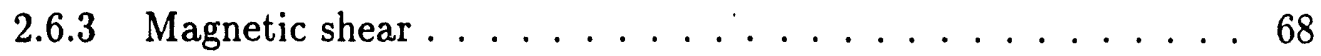

2.6.4 Motion/fluctuation of the cutoff layer . . . . . . . . 70

2.6.5 Fluctuation correlation length $\ldots \ldots \ldots \ldots 71$

2.6.6 Relativistic effects . . . . . . . . . . . . . . 72

2.6.7 Uncertainty in $\omega_{X} \ldots \ldots \ldots \ldots \ldots$

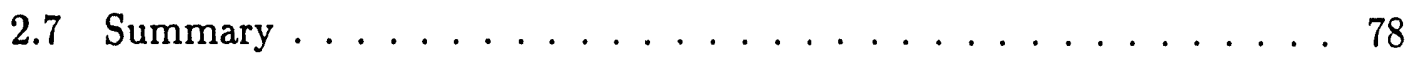

III. Ion Cyclotron Resonance Heating 79

3.1 ICRH vs Other Heating Methods . . . . . . . . . . . . . . 79

3.2 Theory . . . . . . . . . . . . . . . . 81

$3.2 .1 \quad \mathrm{RF}$ heating schemes $\ldots \ldots \ldots \ldots 1$

3.2.2 Multiple species dielectric tensor . . . . . . . . . . . 82

3.3 ICRH and Plasma Edge Interaction $\ldots \ldots \ldots \ldots$. . . . . . 84

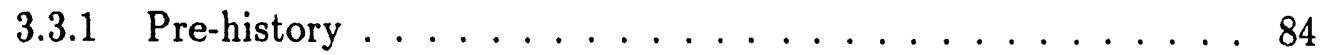

3.3.2 The impurity problem . . . . . . . . . . . . . . . 87

3.3.3 Antenna phasing . . . . . . . . . . . . . . . . 89

3.3.4 The sheath rectification effect $\ldots \ldots \ldots$. . . . 90

3.3.5 Self sputtering and avalanche $\ldots \ldots \ldots \ldots 1$

3.3.6 Increased diffusion at the edge . . . . . . . . . . . 91

3.3.7 Quiver induced losses . . . . . . . . . . . . . . . 92

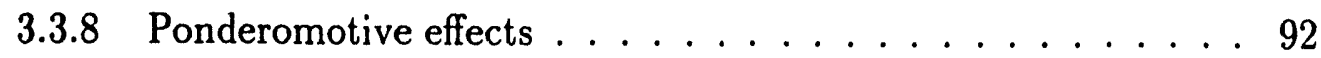

3.4 Antennas in Fusion Experiments . . . . . . . . . . . . . . . . 92

3.5 TFTR Antenna $\ldots \ldots \ldots \ldots \ldots \ldots \ldots$

3.6 Summary . . . . . . . . . . . . . . . . . . 99 
IV. TFTR Reflectometer System

4.1 Development . . . . . . . . . . . . . . . . . . 103

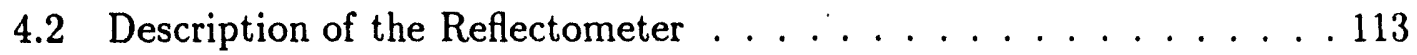

4.3 Major Components of the Reflectometer System . . . . . . . . . 114

4.3 .1 Sources . . . . . . . . . . . . . . . . . . . . . . 114

4.3.2 Directional couplers . . . . . . . . . . . . . . 115

4.4 Summary . . . . . . . . . . . . . . . . . . . 117

$\begin{array}{ll}\text { V. Summary } & 119\end{array}$

\section{APPENDIX}

A. Gaussian Beam Optics 123

A.1 Pre-History of Lasers . . . . . . . . . . . . . . . 123

A.2 Paraxial Ray Analysis . . . . . . . . . . . . . . . . . 127

A.2.1 Ray transfer matrix . . . . . . . . . . . . . 127

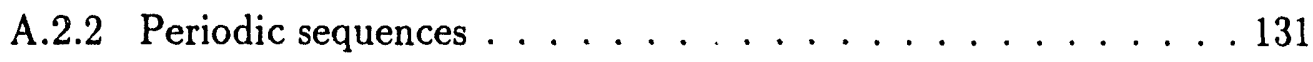

A.3 Wave Analysis of Laser Beams . . . . . . . . . . . . . . 132

A.3.1 Solution to the beam's wave equation $\ldots \ldots \ldots \ldots 132$

A.3.2 Propagation law for the fundamental mode . . . . . . . 134

B. Call Numbers for the Bibliography 138

$\begin{array}{ll}\text { BIBLIOGRAPHY } & 140\end{array}$ 


\section{List of Figures}

1.1 Fusion reactivity as a function of ion temperature. . . . . . . . 3

1.2 Artist's conception of a fusion reaction. . . . . . . . . . . 4

1.3 Schematics of the closed toroidal confinement concepts and a tokamak

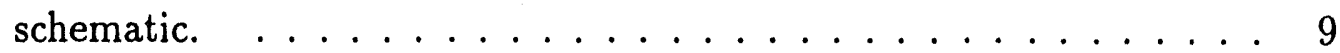

1.4 An artist's sketch of ATF's vacuum chamber and magnetic field coil system. .......................... 10

2.1 CMA diagram with all boundaries and the wave normal surfaces. . . 27

2.2 Plot of the different frequencies for a plasma with a density profile of the type $n_{e}(r) \propto\left(1-\left(\frac{r}{a}\right)^{\alpha}\right)^{\beta}$ and with a magnetic field (in Teslas) of the form $B(R) \propto \frac{1}{R}$, where $r$ is the minor radius, $a$ is the plasma edge minor radius and $R$ is the major radius. DENSO is the center

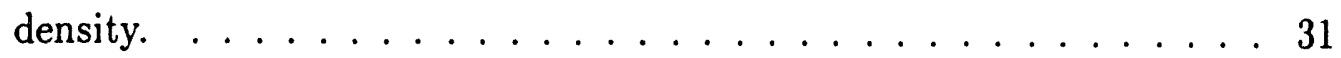

2.3 Generic diagram for an AM reflectometer system. . . . . . . . . 46

2.4 Schematics for PBX-M AM reflectometer system. . . . . . . . . 47

2.5 Basic schematic of a reflectometer. . . . . . . . . . . . 51

2.6 Typical cutoff and resonance frequencies on JT-1. . . . . . . 62

2.7 Reflection and transmission coefficients for a typical TFTR plasma (upper plot). The assumed density profile is included (lower plot) and it is of the from $n_{e} \propto\left(1-\left(\frac{r}{a}\right)^{\alpha}\right)^{\beta}$ where all the parameters have been defined before. ................. 66 
2.8 Density profile for a typical TFTR plasma and corresponding radial

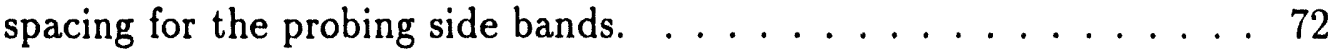

2.9 Relativistic effect on the index of refraction and the location of the cutoff using the weakly relativistic model. $\mu_{r}$ is the refractive index and $T_{e} \approx 10 \mathrm{KeV}$ for plots $a$ to $e . \ldots \ldots \ldots \ldots$

2.10 Comparison of the actual and reconstructed density profile using the weakly relativistic model.

3.1 Typical parabolic density profiles of the form: $\left(1-\left(\frac{r}{a}\right)^{2}\right)^{\alpha}$ and antenna loading vs the profile shape exponent, $\alpha$, and the scrapeoff decay length, $\lambda$. (Batchelor, et al., Fusion Tech., Vol. 21 (1992) p. 1214)

3.2 Loop antenna and circuit representation of both, the short and long loop versions. . . . . . . . . . . . . . . . . . 94

3.3 Long loop antenna used in Alcator-C. . . . . . . . . . . . 95

3.4 Typical cavity antenna and its equivalent circuit. . . . . . . . 96

3.5 Resonant Double Loop antenna and its equivalent circuit. The symmetric version has $\alpha=0.5 \ldots \ldots \ldots \ldots \ldots$. . . . . . . 97

3.6 The U-slot antenna, as proposed by McDonnell Douglas Astronautics

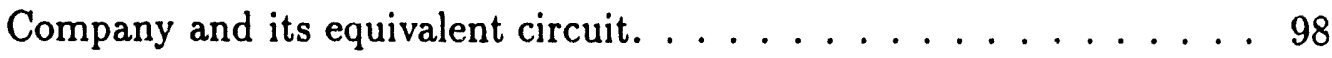

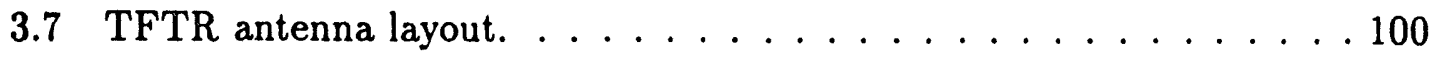

3.8 TFTR antenna cavity box. Most of the Faraday screen elements have been removed to show the current strap housing and the straps

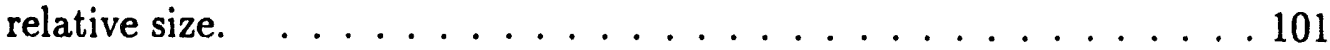

4.1 Reflectometer system design. . . . . . . . . . . . . . . . . . . . 104 
4.2 TFTR plasmas showing the density and toroidal current (magnetic field) range for two different plasma shots. . . . . . . . . . 106

4.3 TFTR fluctuation reflectometer waveguide to vessel interface. . . . . 109

4.4 Layout of the reflectometer for the second phase of the project. . . . 110

4.5 ICRH antenna RF box. This provides a front, side and top view of the antenna. The reflectometer launcher can be seen at the center of the antenna box, front view. The antenna current straps are shown at the top view of the drawing, thus the reflectometer launcher is between the current straps. . . . . . . . . . . . . 111

4.6 Radiation pattern of the launcher in two orthogonal directions, parallel $(\phi=0)$ and perpendicular $(\phi=90)$ to the widest dimension of

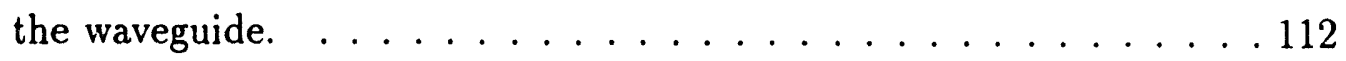

4.7 Plots of the dispersion relations $(\omega=\omega(k))$ for a forward wave (a)

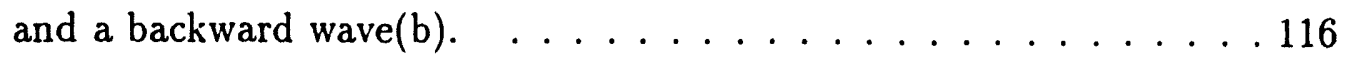

4.8 Backward wave amplifier schematic. $\ldots \ldots \ldots \ldots \ldots$

A.1 TFTR's mirrors setup and geometry. . . . . . . . . . . . . . . 124

A.2 Calculated TFTR's reflectometer launcher Gaussian pattern (unfol-

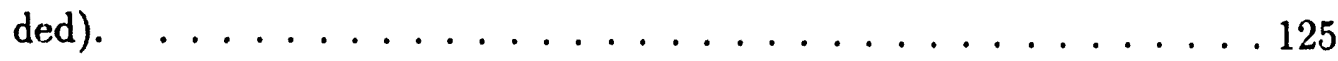

A.3 Calculated TFTR's beam geometry and intersection inside the vacuum chamber. . . . . . . . . . . . . . . 126

A.4 Optical system diagram and parameters. An arbitrary ray and its corresponding parameters are also shown. (C1966 IEEE . . . . . . 129

A.5 Diagram of an infinite sequence of systems (lenses) each characterized by an equal ray transfer matrix. (C1966 IEEE . . . . . . . . . 132 


\section{List of Tables}

1.1 Key plasma parameters: achieved and required. . . . . . . . . . 5

1.2 Some fusion experiments and their corresponding locations. . . . . . 7

1.3 Some stellarators and their corresponding parameters. . . . . . . 8

1.4 Some of ATF major parameters. . . . . . . . . . . . . 11

1.5 Some major parameters of TFTR. . . . . . . . . . . . . 12

1.6 Some of TFTR diagnostics. . . . . . . . . . . . . . 13

3.1 RF experiments list. . . . . . . . . . . . . . . 95

3.2 TFTR antenna design parameters. . . . . . . . . . . . 100

4.1 Some of the digitizer major parameters. . . . . . . . . . . 109

A.1 Ray transfer matrices of six elementary structures. (C)1966 IEEE . . . 130 


\section{CHAPTER I}

\section{INTRODUCTION}

With the recent war in the Persian Gulf the need to reduce our dependance on foreign oil has become strikingly evident. In an attempt to reduce and/or eliminate this dependance the need for new long term energy sources has become obvious. With its almost inexhaustible resources, nuclear fusion has the potential to satisfy our constantly increasing need for inexpensive and clean energy. To reach the goal of nuclear fusion a full understanding of the plasma and transport phenomena must come first. For this purpose the understanding of the main plasma parameters (such as density, temperature, transport coefficients, etc.) is essential.

In this report the principles of reflectometry as a diagnostic tool will be discussed. A novel technique using two wave frequencies with a constant $\delta \omega$ will be discussed, and its potential use for probing the plasma and extracting density profiles under different configurations and plasma conditions will be examined.

This chapter will present an introduction to fusion, reflectometry and a description of the major parameters of the Tokamak Fusion Test Reactor (TFTR) at the Princeton Plasma Physics Laboratory in Princeton, New Jersey.

\section{$1.1 \quad$ Fusion}

As far as is known, fusion is the primary source of energy in the universe. Nuclear fusion is the process in which two light nuclei (the parents) join together 
to form a new nucleus (daughter or products) of bigger mass than the individual parent nuclei, but with nuclear mass slightly smaller (for exothermic reactions) than the combined mass of the parents. The mass difference is transformed into kinetic energy ${ }^{1}$ (since mass is energy, as proved by Einstein with his famous formula $E=$ $\left.m c^{2}\right)$ and distributed among the daughters of the fusion reaction. Fusion reactions in which energy is released (exothermic reactions) can occur between elements that are below iron ( $\mathrm{Fe})$ in the periodic table. Fusion of elements heavier than $\mathrm{Fe}$ is generally endothermic. This is because $\mathrm{Fe}$ has the lowest energy per nucleon in the periodic Table. ${ }^{2}$ This explains why fission of heavy elements such as ${ }^{235} \mathrm{U}$ is exothermic.

Hydrogen is the most abundant element in the universe. This and other characteristics (such as reactivity, energy released per reaction, etc.) make it an excellent fuel source for a fusion reactor. To accomplish fusion, the positively charged nuclei most overcome the Coulomb repulsive electrostatic force and get close enough so that the attractive nuclear forces can take action and fuse the nuclei permarently together. From the hydrogen family the two best candidates for fusion fuel are Deuterium $\left({ }_{1}^{2} \mathrm{D}\right)$ and Tritium $\left({ }_{1}^{3} \mathrm{~T}\right)$, mainly because of their high fusion reactivity at low temperature when compared with other elements, as can be seen from Figure 1.1. ${ }^{1}$ The fusion reactions of most interest using hydrogen isotopes are: ${ }^{3}$

$$
\begin{aligned}
& { }_{1}^{2} \mathrm{D}+{ }_{1}^{3} \mathrm{~T} \rightarrow{ }_{2}^{4} \mathrm{He}(3.45 \mathrm{MeV})+{ }_{0}^{1} \mathrm{n}(14.15 \mathrm{MeV}) \\
& { }_{1}^{2} \mathrm{D}+{ }_{1}^{2} \mathrm{D} \rightarrow{ }_{1}^{3} \mathrm{~T}(1.01 \mathrm{MeV})+{ }_{1}^{1} \mathrm{p}(3.02 \mathrm{MeV}) \\
& { }_{1}^{2} \mathrm{D}+{ }_{1}^{2} \mathrm{D} \rightarrow{ }_{2}^{3} \mathrm{He}(0.82 \mathrm{MeV})+{ }_{0}^{1} \mathrm{n}(2.45 \mathrm{MeV}) \\
& { }_{1}^{2} \mathrm{D}+{ }_{2}^{3} \mathrm{He} \rightarrow{ }_{2}^{4} \mathrm{He}(3.67 \mathrm{MeV})+{ }_{1}^{1} \mathrm{p}(14.67 \mathrm{MeV})
\end{aligned}
$$

and the approach for equation 1.1 is shown in Figure 1.2.

A secondary advantage of the D-T reaction is that about $20 \%$ of the energy goes to the Helium (He) ion which can be confined in the plasma and helps to heat 
1.1: Fusion

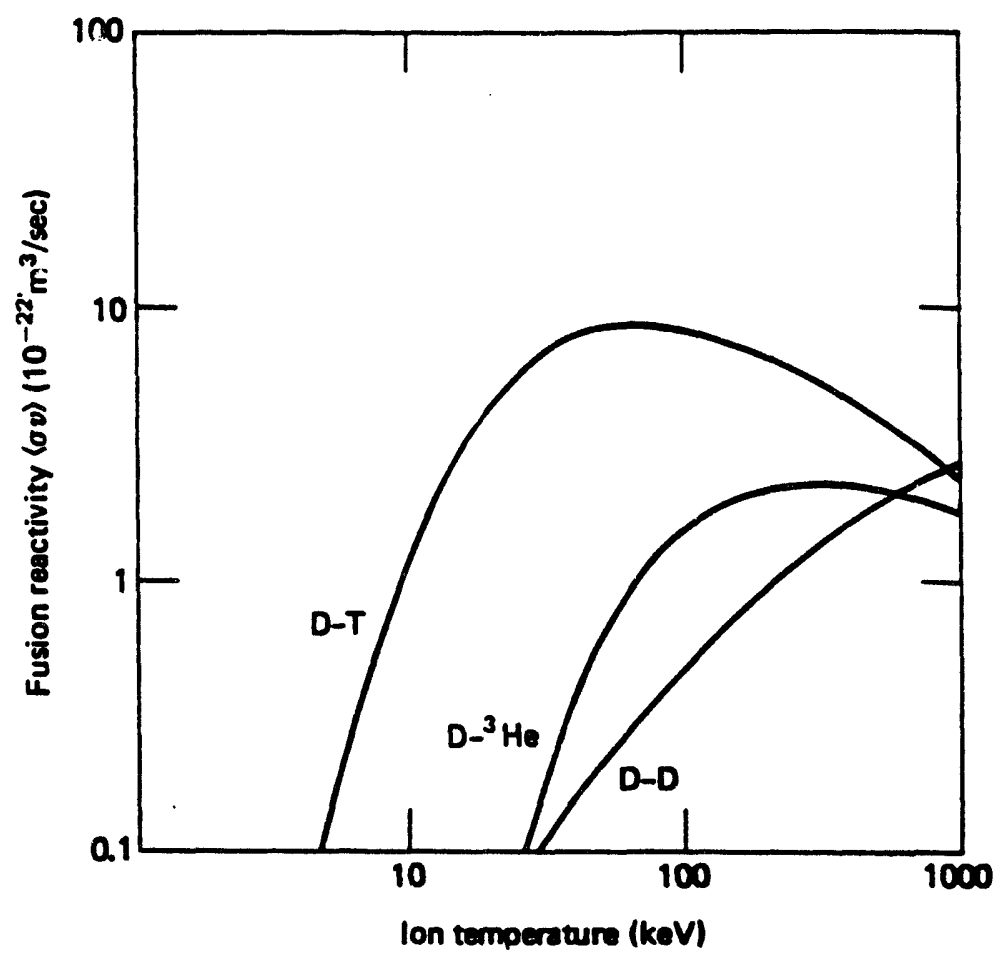

Figure 1.1: Fusion reactivity as a function of ion temperature. 


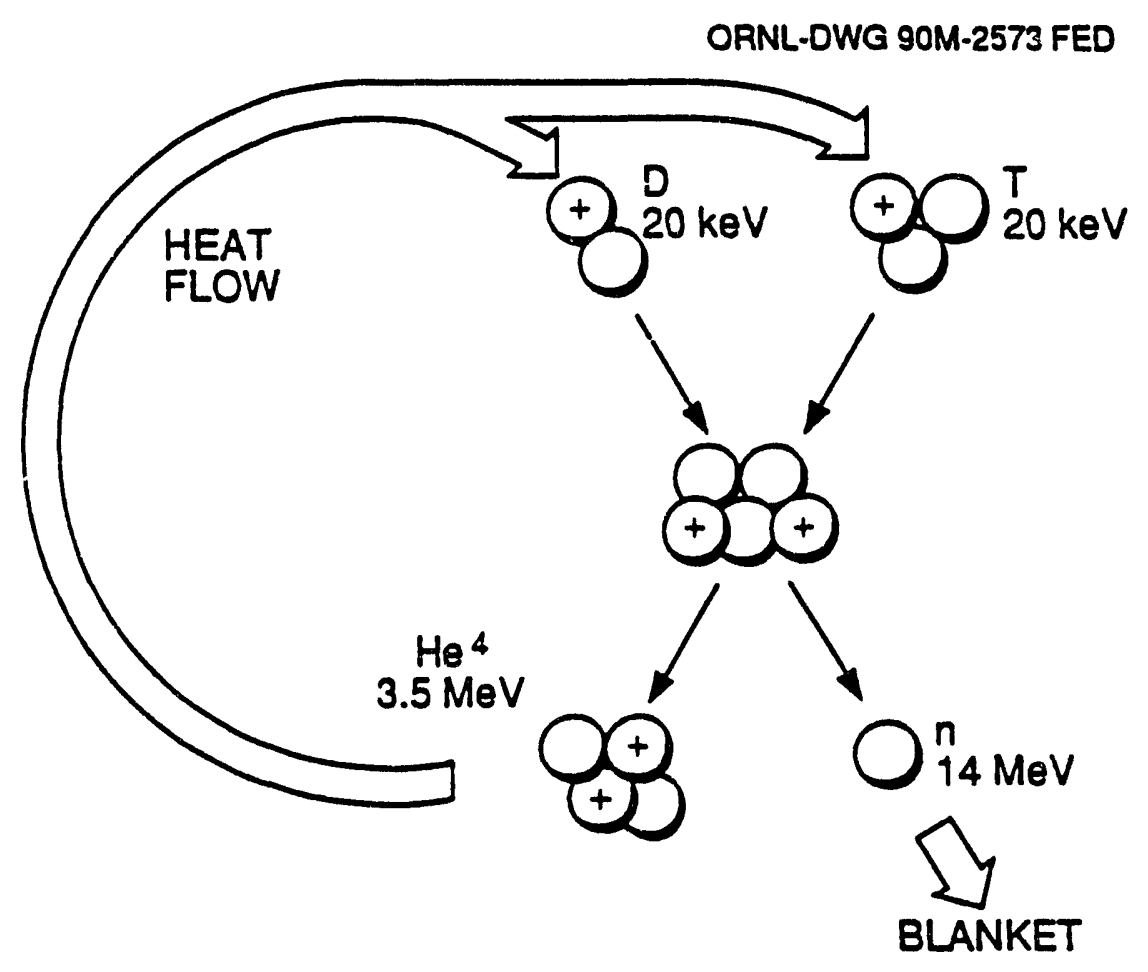

Figure 1.2: Artist's conception of a fusion reaction. 


\begin{tabular}{|c|c|c|c|c|}
\hline Parameter & 1971 & 1981 & 1991 & Steady State \\
\hline $\begin{array}{c}\text { Central ion temp. } \\
T_{i 0}(\mathrm{KeV})\end{array}$ & 0.5 & 7.0 & 35 & 30 \\
\hline $\begin{array}{l}\text { Central electron temp. } \\
T_{e 0}(\mathrm{KeV})\end{array}$ & 1.5 & 3.5 & 15 & 30 \\
\hline $\begin{array}{l}\text { Energy confinement time } \\
\tau_{E}(\mathrm{sec})\end{array}$ & 0.007 & 0.02 & 1.4 & 3.0 \\
\hline $\begin{array}{l}\text { Triple product } \\
\qquad n_{i 0} T_{i 0} \tau_{E}\left(\mathrm{KeV} \frac{\text { sec }}{m^{3}}\right)\end{array}$ & $1.5 \times 10^{17}$ & $5.5 \times 10^{18}$ & $9.0 \times 10^{20}$ & $7.0 \times 10^{21}$ \\
\hline $\begin{array}{l}\text { Plasma pressure } \\
\qquad \beta=\frac{2 \mu_{0} \bar{p}}{B^{2}}\end{array}$ & $0.1 \%$ & $3 \%$ & $11 \%$ & $5 \%$ \\
\hline $\begin{array}{l}\text { Current-drive parameter } \\
\qquad \eta_{C D}=\frac{n_{e 0} R I_{P}}{P_{C D}}\left(\frac{A}{m^{2} W}\right)\end{array}$ & - & $8 \times 10^{18}$ & $\begin{array}{r}3.4 \times 10^{19} \\
+ \text { bootstrap }\end{array}$ & $\begin{array}{r}3 \times 10^{19} \\
+ \text { bootstrap }\end{array}$ \\
\hline $\begin{array}{l}\text { Fusion reactivity: } \\
\text { D-D reactions } / \mathrm{sec} \\
\mathrm{D}-\mathrm{T} \text { reactions } / \mathrm{sec}\end{array}$ & - & $\begin{array}{c}3 \times 10^{14} \\
-\end{array}$ & $\begin{array}{l}1 \times 10^{17} \\
\approx 6 \times 10^{17}\end{array}$ & $\overline{10^{21}}$ \\
\hline
\end{tabular}

Table 1.1: Key plasma parameters: achieved and required.

the plasma. The remaining $80 \%$ of the energy goes to the neutron, which has no net charge, so it leaves the plasma and deposits its energy in the vacuum vessel and/or neutron blanket. The energy deposited in the blanket can be used to make steam and drive a standard steam power plant. To sustain the D-T reaction, the plasma and energy necessary for the reaction must be contained in the vacuum vessel for a reasonable length of time. Some key plasma parameters for the achieved and required values are shown in Table 1.1. ${ }^{3}$ To this day, anomalous transport, short energy confinement times, and some engineering difficulties have prevented us from developing fusion to its full potential. The goal of the fusion program is to overcome all these problems and develop a commercially attractive fusion reactor. 


\section{$1.2 \quad$ Reflectometry}

Reflectometry is a technique developed during the 1960s and works on the same principle as the RADAR (RAdio Detection And Ranging). That is, it measures the distance (position) between a transmitter (source) and a target (plasma) using

the delay time (phase difference) between the incident and reflected wave. ${ }^{5-8} \mathrm{~A}$ more detailed history of the development of reflectometry is offered in section 2.1 beginning on page 14 .

\subsection{Fusion Experiments}

Presently magnetic fusion research takes place mainly in relatively large machines of three main types: Tokamaks, Stellarators and Torsatrons. There are other less popular configurations ${ }^{9}$ like the Symmotron, the modular stellarator, heliac (a type of helical axis stellarator), the spheromak, etc. ${ }^{14-16}$ but they will not be discussed here. Table 1.2 shows a list of fusion experiments and their corresponding location. ${ }^{3}$ The most common and popular of these three machines is the tokamak.

These machines rely on a helical magnetic field to confine the hot plasma to achieve fusion. What differentiates them is the way in which they achieve this helical magnetic field. The tokamak uses a set of toroidal field coils to produce the toroidal component of the helical magnetic field and a transformer set of coils to induce a current in the plasma that produces the poloidal component of the helical magnetic field. ${ }^{1}$ A simplified schematic is presented in Figure $1.3,{ }^{1}$ top picture, together with a more detailed diagram of a fusion experiment showing the helical magnetic field line through the cutout section, which is a product of the vectorial addition of the Poloidal and Toroidal magnetic fields. The stellarator and torsatron are characterized by a set of external helical coils that produce the magnetic field 


\begin{tabular}{||l|l|l||}
\hline Device & Status & Location \\
\hline Alc-C-M & On & Alcator-C Modified, Plasma Fusion Center, MIT \\
\hline PLT & Off & Princeton Large Tokamak, Princeton Plasma Phy. Lab. \\
\hline ATC & Off & Adiabatic Toroidal Compressor, PPPL \\
\hline PBX-M & On & Princeton Divertor Experiment, PPPL \\
\hline TFTR & On & Tokamak Fusion Test Reactor, PPPL \\
\hline BPX & & Burning Plasma Exp., PPPL (proposed) \\
\hline DIII-D & On & Doublet III-D, General Atomics, San Diego, Ca. \\
\hline ORMAK & Off & Oak Ridge Tokamak, Oak Ridge Nat. Lab. \\
\hline ISX-B & Off & Impurity Studies Experiment B, ORNL \\
\hline ATF & Off & Advanced Toroidal Facility, ORNL \\
\hline ITER & & Inter. Thermonuclear Exp. Reactor (proposed) \\
\hline AsDEX-U & On & $\begin{array}{l}\text { Axially Symmetric Divertor Experiment, Max Planck } \\
\text { Institute for Plasma Physics, Garching, Germany }\end{array}$ \\
\hline Textor & On & Jülich, Federal Republic of Germany \\
\hline JET & On & Joint European Torus, Abingdon, England \\
\hline JFT-2M & On & Japan Atomic Energy Research Ins., Takai, Japan \\
\hline JT-60U & On & Japan Atomic Energy Research Ins., Naka, Japan \\
\hline T-3 & On & Kurchatov Institute, Moscow \\
\hline T-10 & On & Kurchatov Institute, Moscow \\
\hline TFR & On & Centre d'Etudes Nucleaire, Fontanay-aux-Roses, France \\
\hline
\end{tabular}

Table 1.2: Some fusion experiments and their corresponding locations. 


\begin{tabular}{||l|l|l|l|l|l|l|l|l||}
\hline Device & Location & $\begin{array}{l}\text { Start } \\
\text { Date }\end{array}$ & $\mathrm{l}$ & $\mathrm{m}$ & $\begin{array}{l}\mathrm{R} \\
(\mathrm{m})\end{array}$ & $\begin{array}{l}\mathrm{a} \\
(\mathrm{m})\end{array}$ & $\begin{array}{l}\mathrm{B} \\
(\mathrm{T})\end{array}$ & $\begin{array}{l}\text { Main } \\
\text { Feature }\end{array}$ \\
\hline ATF & USA & 1987 & 2 & 12 & 2.1 & 0.27 & 1.9 & Flexibility \\
CAT & USA & 1990 & 1,2 & 5 & 0.53 & 0.11 & 0.1 & Torsatron \\
IMS & USA & 1989 & 3 & 7 & 0.4 & 0.04 & 0.3 & Modular Stel. \\
L-2 & Russia & 1976 & 2 & 14 & 1.0 & 0.11 & 1.5 & Stellarator \\
URAGAN-2 & Ukraine & 1993 & 2,4 & 4 & 1.7 & 0.2 & 2.4 & Torsatron \\
URAGAN-3 & Ukraine & 1982 & 3 & 9 & 1.0 & 0.13 & 1.2 & Divertor \\
Wend. 7-A & FRG & 1976 & NA & 5 & 2.0 & 0.1 & 3.5 & Shearless \\
Wend. 7-AS & FRG & 1987 & NA & 5 & 2.0 & 0.2 & 2.5 & Modular coils \\
Wend. 7-X & FRG & $200 ?^{*}$ & NA & 5 & 5.5 & 0.5 & 2.5 & Advance Stel. \\
Heliotron-E & Japan & 1981 & 2 & 19 & 2.2 & 0.2 & 1.9 & High $\bar{\imath}$ shear \\
CHS & Japan & 1988 & 2 & 8 & 1.0 & 0.2 & 2.0 & Heliotron \\
LHD & Japan & 1997 & 2 & 10 & 3.9 & 0.65 & 3.0 & Heliotron \\
TJ IU & Spain & 1994 & 1 & 6 & 0.6 & 0.1 & 0.5 & Torsatron \\
TJ-II & Spain & 1996 & 1 & 4 & 1.5 & 0.25 & 1.0 & Helical axis \\
H-1 & Australia & 1993 & 1 & 3 & 1.0 & 0.2 & 1.0 & Flexible Heliac \\
\hline
\end{tabular}

Table 1.3: Some stellarators and their corresponding parameters.

with no internal plasma current. ${ }^{9}$ The stellarator has helical coils with alternating antiparallel currents that produce a net poloidal magnetic field and no net toroidal field. Because of the zero net toroidal field, a separate set of toroidal field coils is used to provide the toroidal component of the helical magnetic field. A list of some major stellarators $^{9-11}$ is shown in Table 1.3 with their corresponding main parameters. On the torsatron (a subclass of the stellarator) the currents run parallel to each other, creating poloidal and toroidal magnetic fields; thus, no extra toroidal field coils are needed, but vertical field coils are used to provide stabilization and positioning of the plasma. An example of a torsatron is the Advanced Toroidal Facility (ATF) at the Oak Ridge National Laboratory, shown in Figure $1.4 .^{9}$ Some of its main parameters are listed in Table 1.4.

\footnotetext{
*If things go well, start date maybe some time after 2000.
} 

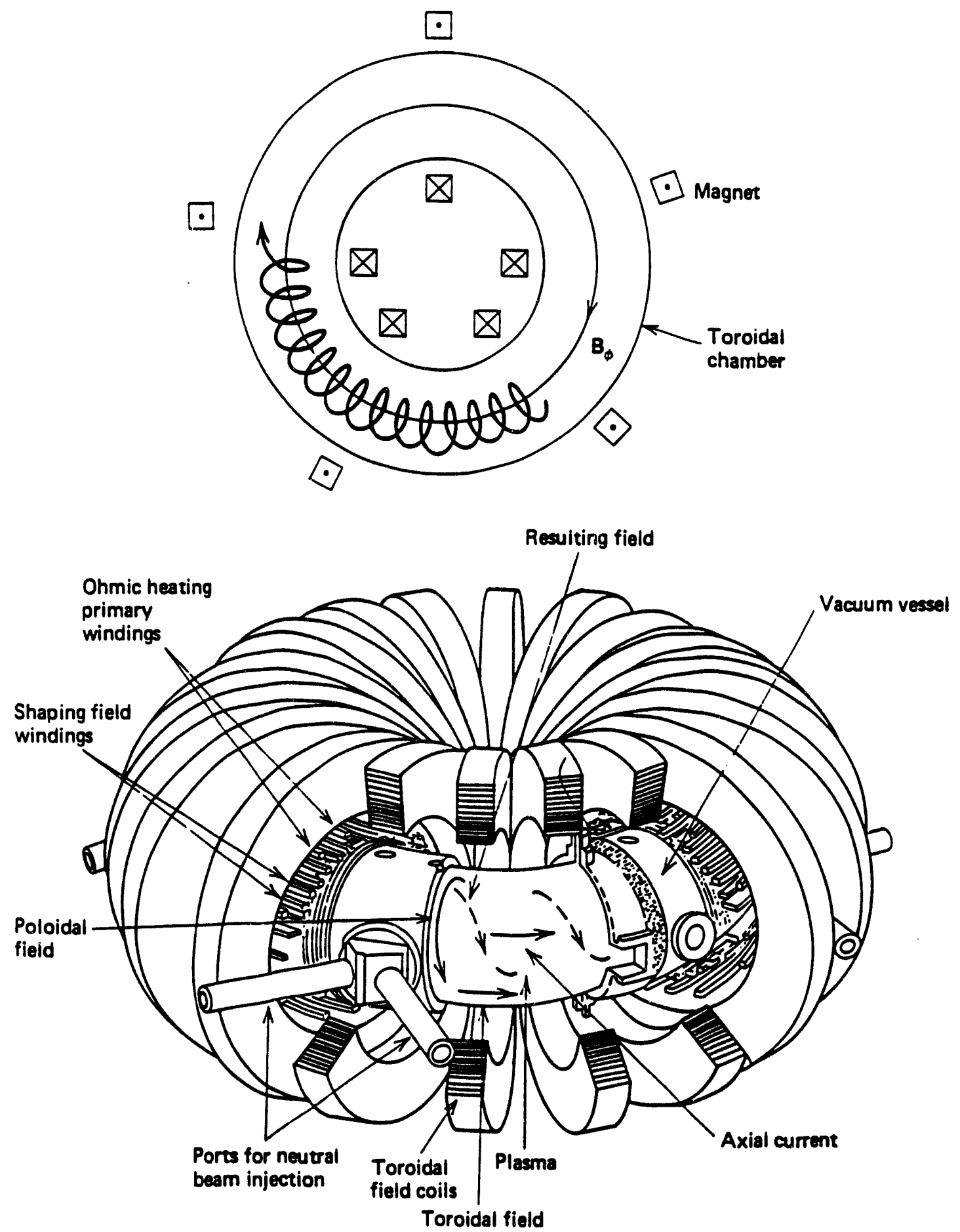

Figure 1.3: Schematics of the closed toroidal confinement concepts and a tokamak schematic. 


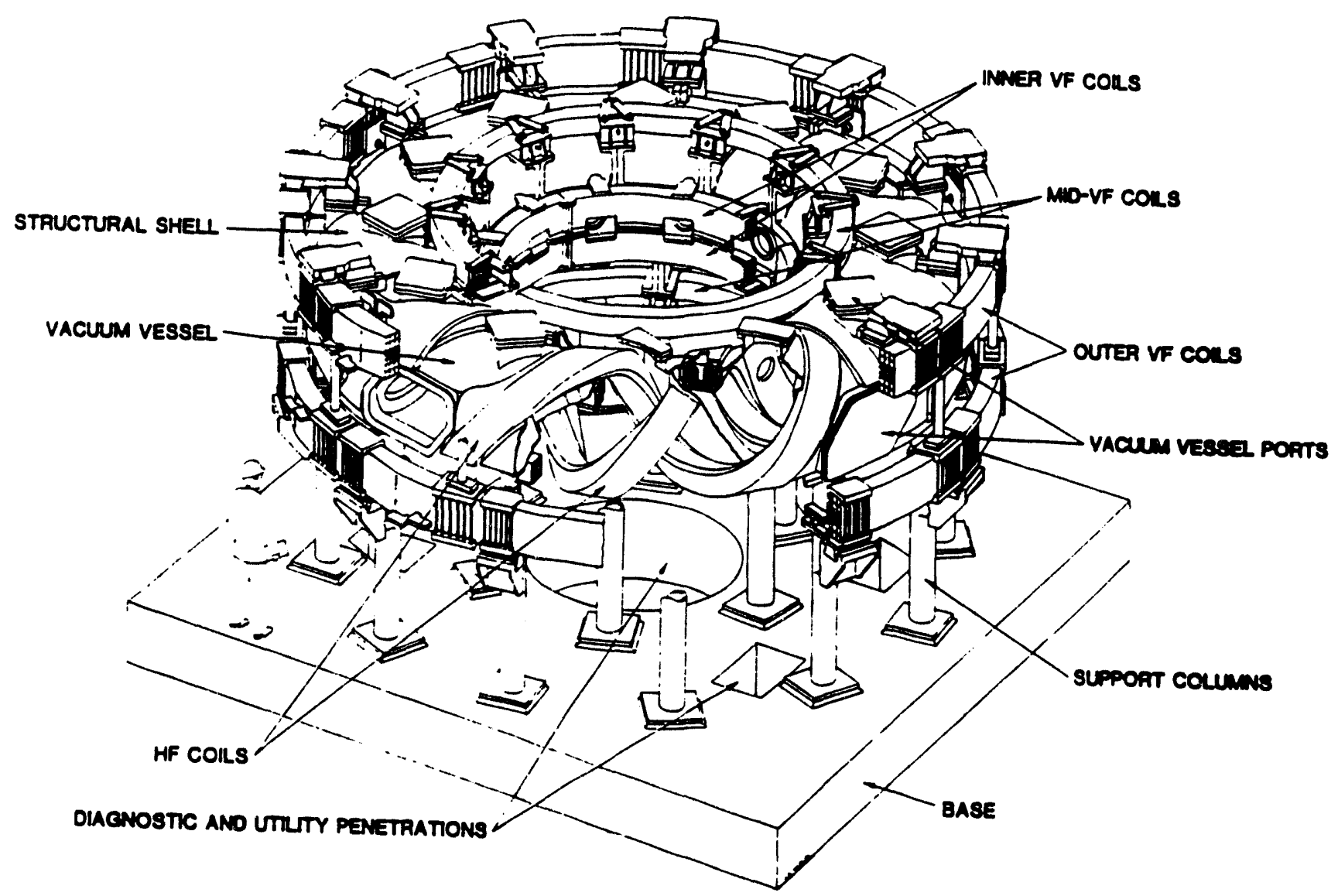

Figure 1.4: An artist's sketch of ATF's vacuum chamber and magnetic field coil system. 


\begin{tabular}{|c|c|c|}
\hline \multirow[t]{4}{*}{ Dimensions } & Major radius, $R_{0}$ & $2.1 \mathrm{~m}$ \\
\hline & Minor radius, $a$ & $0.27 \mathrm{~m}$ \\
\hline & Plasma volume, $V_{p}$ & $3.0 \mathrm{~m}^{3}$ \\
\hline & Vacuum vessel volume & $10.5 \mathrm{~m}^{3}$ \\
\hline \multirow{7}{*}{$\begin{array}{l}\text { Magnetic } \\
\text { configuration }\end{array}$} & Maximum field on axis, $B_{0}$ & 1.9 Teslas \\
\hline & Field flattop time at $1.9 \mathrm{~T}$ & $5 \mathrm{sec}$ \\
\hline & Field flattop time at $0.95 \mathrm{~T}$ & $20 \mathrm{sec}$ \\
\hline & Poloidal multipolarity, $l$ & 2 \\
\hline & Number of field periods, $m$ & 12 \\
\hline & Standard central transform, $\bar{\imath}(0)$ & 0.3 \\
\hline & Standard edge transform, $\bar{\imath}(a)$ & 1.0 \\
\hline \multirow{3}{*}{$\begin{array}{l}\text { Plasma } \\
\text { Heating }\end{array}$} & $\mathrm{ECH}, 53 \mathrm{GHz}$, steady state & $0.4 \mathrm{MW}$ \\
\hline & NBI, $30 \mathrm{kv}, 0.3 \mathrm{sec}$ & $2 \mathrm{MW}$ \\
\hline & $\mathrm{ICH}, 29 \mathrm{MHz}, 0.1 \mathrm{sec}$ & $1.0 \mathrm{MW}$ \\
\hline
\end{tabular}

Table 1.4: Some of ATF major parameters.

\subsection{Tokamak Fusion Test Reactor}

The TFTR experiment, originally conceived in 1974, became operational in December 1982. The original basic objectives of TFTR are the following: ${ }^{12}$

1. to achieve temperatures $(10 \mathrm{KeV})$ and densities $\left(10^{14} \frac{\mathrm{part}}{\mathrm{cm}^{3}}\right)$ pertinent to a fusion reactor in plasmas with currents of 2.5 MAmps

2. to achieve approximate energy break-even between the power input to and neutron power output from the plasma $(Q \approx 1)$ at reactor-like fusion power densities $\left(\approx 1 \frac{\mathrm{Watts}_{\mathrm{cm}}}{\mathrm{cm}^{3}}\right)$.

The TFTR program goals are to understand plasma transport in reactor relevant regimes, achieve conditions in deuterium plasmas that project to energy breakeven in D-T plasmas, carry out physics research and development for TPX (Tokamak Physics eXperiment) and ITER (International Thermonuclear Experimental Rector), and achieve breakeven conditions in D-T plasmas. Some machine 


\begin{tabular}{||l|r||}
\hline Major radius, $R_{0}$ & $2.63 \mathrm{~m}$ \\
\hline Minor radius, $a$ & $0.97 \mathrm{~m}$ \\
\hline Plasma volume, $V_{p}$ & $46 \mathrm{~m}^{3}$ \\
\hline Toroidal magnetic field, $B_{0}$ & $4.9 \mathrm{~T}$ \\
\hline Plasma current $\left(\right.$ maximum), $I_{p}$ & $3 \mathrm{MA}$ \\
\hline Plasma density, $n_{e}$ & $5 \times 10^{19} \frac{\mathrm{part}^{3}}{\mathrm{~m}^{3}}$ \\
\hline Neutral beam power, $P_{N B I}$ & $35 \mathrm{MW}$ \\
\hline ICRH power, $P_{I C R H}$ & $12 \mathrm{MW}$ \\
\hline
\end{tabular}

Table 1.5: Some major parameters of TFTR.

parameters are shown in Table $1.5,{ }^{4,13}$ and some diagnostics are presented in Table $1.6^{12,13}$ (a very good article is the one by Orlinskij). 


\begin{tabular}{|l|l|r|r|}
\hline Diagnostic & Measurement & $\begin{array}{r}\text { \# Data } \\
\text { Channels }\end{array}$ & $\begin{array}{r}\text { Data } \\
\text { Points }\end{array}$ \\
\hline 1 mm Interferometer & $\int n_{e} d l$ & 2 & $32 \mathrm{~K}$ \\
\hline Torus Pressure Gauges & Torus pressure & 2 & $8 \mathrm{~K}$ \\
\hline Residual Gas Analysis & Gas impurity concentration & 2 & - \\
\hline Rogowski Loops & $I_{p}$ & 4 & $32 \mathrm{~K}$ \\
\hline Position Coils & Plasma position & 37 & $108 \mathrm{~K}$ \\
\hline Mirnov Coils & Magnetic oscillations & 43 & $195 \mathrm{~K}$ \\
\hline Voltage Loops/Saddle Coils & Vol. around torus, position & 44 & $88 \mathrm{~K}$ \\
\hline Diamagnetic Loop & Plasma pressure & 4 & $4 \mathrm{~K}$ \\
\hline Hard X-ray Monitors & Effects of runaway electrons & 5 & $40 \mathrm{~K}$ \\
\hline Plasma TV & Plasma pictures, position & 1 & - \\
\hline Fast Scan. Het. Radiometer & $T_{e}(r, t)$ & 2 & $32 \mathrm{~K}$ \\
\hline X-ray Pulse-height Analyzer & $T_{e}, n_{F e}, Z_{e f f}$ & 9 & $102 \mathrm{~K}$ \\
\hline Horizontal Charge Exchange & $T_{i}(t)$ & 198 & $198 \mathrm{~K}$ \\
\hline $\begin{array}{l}\text { 235 U, BF } \\
\text { Pr }{ }^{3} \text { He Neutron }\end{array}$ & Neutrons & 12 & $44 \mathrm{~K}$ \\
\hline Proportional Counters & $T_{i}(t)$ & 34 & $34 \mathrm{~K}$ \\
\hline Bolometers & Radiated (and C-EX) power & 2 & $64 \mathrm{~K}$ \\
\hline UV Survey Spectrometer & Impurity Spectrum $(t)$ & 64 & $16 \mathrm{~K}$ \\
\hline Interference Filter Array & $Z_{e f f}(t), H_{\alpha}$ light $(t), n_{e}(r, t)$ &
\end{tabular}

Table 1.6: Some of TFTR diagnostics. 


\section{CHAPTER II}

\section{REFLECTOMETRY}

This chapter presents a short history of the beginnings of reflectometry, and describes its basic principles, the propagation of electromagnetic waves in fusion plasmas, and the different reflectometry techniques.

\subsection{History of Reflectometry}

\subsubsection{The beginings}

Since the 1960's research programs in fusion, ionospheric physics, and astrophysics have led to developments in the study of plasma physics. Reflectometry has its origins in the field of ionospheric physics,,$^{5,6,8}$ in which the altitude (position) of an atmospheric density layer is calculated from the delay time of an electromagnetic wave after it is reflected off a plasma layer (the cutoff layer) for which the electron plasma frequency $\left(\omega_{p e}^{2}=\frac{q_{e}^{2} n_{e}}{c_{0} m_{e}}\right)$ is equal to the wave frequency $\omega$. Reflectometry works under the same principle than the famous $R A D A R$ principle (RAdio Detection And Ranging), except that the reflectometer is reflecting from a plasma and not from a solid object. For the particular case of laboratory plasmas, the time delays are so small (of the order of nanoseconds) that the phase difference between the incident and the reflected waves is measured, rather than the time delay. 


\subsubsection{First experiments in Russia}

The first use of reflectometry on fusion plasmas was performed in what at that time was known as the Union of Soviet Socialist Republics (USSR) and now consists of many different and independent nations ${ }^{17,18}$ (now frequently refered to as the "Former Soviet Union" or the Soviet DisUnion). In this experiment Anisimov used three sources of microwaves to probe the plasma at three different densities. It was a very simple method compared to today's technology, but it made a proof of principle of the potentials of the technique. Many others, such as Colchin, ${ }^{19,20}$ Simonet, ${ }^{21}$ Anoshkin, ${ }^{18}$ Calderon, ${ }^{25}$ Sánchez, ${ }^{26-32}$ etc. followed up on the idea and developed it to its present more sophisticated state.

\subsubsection{First experiment in USA}

The first reflectometry (underground) experiment in the United States was done by Colchin ${ }^{19,20}$ in ORMAK and as he explains in his report "... it cost almost nothing. The equipment was entirely borrowed; setup, and most of the runs were made after hours. Hence the 'underground' in the title." They used a frequency swept microwave source in an attempt to make time resolved density profiles. The success of this effort indicated that the microwave sweeper held out the promise of detailed time-resolved density measurements on a shot-to-shot basis.

Since that time, reflectometry at fixed frequencies, narrowband swept frequencies, and broad band swept frequencies has been used in many fusion experiments over the world. For example, reflectometry at fixed frequencies has been used on the ATC tokamak, ${ }^{37}$ on the TORSO stellarator, ${ }^{42}$ on JET, ${ }^{136}$ on TFTR $^{100-102,111,105-108}$ and on TFR $^{76}$ to measure density fluctuations. Reflectometry over a narrow range was used on TFR to follow the lateral movement of the discharge and to uetermine when a particular density was reached in the plasma ${ }^{77}$. and on PLT to control auxiliary high-power plasma heating systems. ${ }^{79}$ Broadband 
reflectometry was used on DIII-D to measure density profiles of the plasma ${ }^{93-95}$ and density fluctuations. ${ }^{93}$

\subsection{Propagation of Electromagnetic Waves in Plasmas}

This section will present a brief derivation of the equations that describe the propagation of electromagnetic waves in plasmas with an emphasis on fusion plasmas.

\subsubsection{Maxwell equations}

No paper about electromagnetism can be complete without a presentation of Maxwell's equations. This section will present some important equations and some definitions that are the basis of electromagnetism and most of the present work.

Maxwell's equations describe the propagation of electromagnetic waves in a medium (or plasma). These are (in MKS units):48

$$
\begin{aligned}
\vec{\nabla} \cdot(\overline{\bar{\epsilon}} \cdot \vec{E}) & =\rho & & \text { (Gauss' electric field law) } \\
\vec{\nabla} \cdot \vec{B} & =0 & & \text { (Gauss' magnetic field law) } \\
\vec{\nabla} \times \vec{E} & =-\frac{\partial \vec{B}}{\partial t} & & \text { (Faraday's induction law) } \\
\vec{\nabla} \times \vec{B} & =\mu_{0}\left(\vec{J}+\frac{\partial(\overline{\bar{\epsilon}} \cdot \vec{E})}{\partial t}\right) & & \text { (generalized Ampére's law) }
\end{aligned}
$$

where:

1. $\vec{E}=$ Electric vector field in Volts/meter.

2. $\vec{D}=$ Electric displacement vector field $=\overline{\bar{\epsilon}} \cdot \vec{E}$ in Coulombs $/ \mathrm{meter}^{2}$, where $\overline{\bar{\epsilon}}$ is the permittivity (dielectric tensor) of the medium. 
3. $\vec{B}=$ Magnetic induction vector field $=\mu \vec{H}$ in Teslas, where $\mu$ is the permeability of the medium.

4. $\vec{H}=$ Magnetic field in Ampère-turn/meter.

5. $\rho=$ Electrical charge density in Coulombs/meter ${ }^{3}$.

6. $\vec{J}=$ Electrical current density vector field in Ampère/meter ${ }^{2}$.

7. $c=299,792,456.2 \pm 1.1$ meters $/$ second.${ }^{50}$

Among the consequences of Maxwell equations, there is the continuity equation:

$$
\vec{\nabla} \cdot \vec{J}+\frac{\partial \rho}{\partial t}=0
$$

which is derived by taking the divergence $(\vec{\nabla} \cdot)$ of equation 2.4 above.

A useful quantity in plasma studies is the electric conductivity tensor, $\overline{\bar{\sigma}}$, defined by:

$$
\vec{J} \equiv \overline{\bar{\sigma}} \cdot \vec{E}
$$

which relates the current density, $\vec{J}$, to the applied external electric field in an anisotropic medium.

The polarization vector field, $\vec{P}$, is another quantity of importance, defined together with the susceptibility tensor, $\overline{\bar{\chi}}$, as:

$$
\vec{P} \equiv \epsilon_{0} \overline{\bar{\chi}} \cdot \vec{E}
$$

and it represents the reaction of the medium (plasma) to the external applied electric field. Among the conveniences of defining the polarization vector and electric susceptibility is that it provides us with a divergence-free electric displacement field in the medium, that is:

$$
\vec{\nabla} \cdot \vec{D}=0
$$


where:

$$
\vec{D} \equiv \epsilon_{0} \vec{E}+\vec{P}
$$

where by using eq. 2.7 one obtains:

$$
\vec{D}=\epsilon_{0} \vec{E}+\epsilon_{0} \overline{\bar{\chi}} \cdot \vec{E}
$$

and since:

$$
\vec{D}=\overline{\bar{\epsilon}} \cdot \vec{E}
$$

then:

$$
\overline{\bar{\epsilon}}=\epsilon_{0}(\overline{\bar{I}}+\overline{\bar{\chi}})
$$

All of this leads to the polarization charge density:

$$
\rho=-\vec{\nabla} \cdot \vec{P}
$$

\subsubsection{The dielectric tensor}

The propagation of an electromagnetic wave in a plasma can be described and/or modeled by the dielectric tensor, which represents a measurement of the response of a dielectric medium to an externally applied electromagnetic field. For such a model it will be assumed that: $5,8,48,51-65,132$

1. The fields and the velocities are Fourier transformable.

2. The velocity of the species are all the same. This means that the particles have no thermal motion.

This description addresses what is known as the cold plasma model; and even though the model is very simple, these idealizations capture, to a large extent, the main characteristics of a plasma response to an external electromagnetic wave. The term "cold" is used to indicate that the electrons and ions have no thermal 
velocities, except the one that it is induced by the electromagnetic fields of the wave and the natural gyration motion of the electrons. More details on the dielectric tensor can be found in the references, such as D. G. Swanson, ${ }^{48}$ T. H. Stix, ${ }^{53-55}$ W. P. Allis, ${ }^{52}$ H. Meuth, ${ }^{132}$ K. G. Budden, ${ }^{5}$ M. Bornatici, ${ }^{51}$ V. I. Ginzburg ${ }^{8}$ and others.

Next consider a particle of mass $m$, and charge $q$, that moves with velocity $\vec{v}$. under the influence of the Lorentz force in an electric field $\vec{E}$ and magnetic field $\vec{B}: 5,48,51-55,132,8$

$$
\frac{d \vec{v}_{o}}{d t}=\frac{q_{0}}{m_{0}}\left(\vec{E}+\vec{v}_{0} \times \vec{B}\right)
$$

along with two of Maxwell's equations:

$$
\begin{aligned}
\vec{\nabla} \times \vec{E} & =-\frac{\partial \vec{B}}{\partial t} \\
\vec{\nabla} \times \vec{B} & =\mu_{0}\left(\vec{J}+\epsilon_{0} \frac{\partial \vec{E}}{\partial t}\right)
\end{aligned}
$$

where the term $\epsilon_{0} \frac{\partial \vec{E}}{\partial t}$ is known as the displacement current of the externally applied electric field. The equation for the current density is given by:

$$
\vec{J}=\sum_{\bullet} n_{\imath} q_{v} \vec{v}
$$

which includes all the species of the plasma and it assumes that the plasma is locally uniform and homogenous. For the study of the oscillatory properties of the plasma, it can be assumed that the $\vec{E}, \vec{B}$ and $\vec{v}$, fields can be Fourier transformed and that they have the form:

$$
\begin{aligned}
\vec{E}(\vec{r}, t) & =\vec{E} e^{i(\vec{k} \cdot \vec{r}-\omega t)} \\
\vec{B}(\vec{r}, t) & =\vec{B}_{0}+\vec{B} e^{i(\vec{k} \cdot \vec{r}-\omega t)} \\
\vec{v}_{0}(\vec{r}, t) & =\vec{v}_{0} e^{i(\vec{k} \cdot \vec{r}-\omega t)}
\end{aligned}
$$

where $\vec{B}_{0}$ is the static part of the magnetic field and it is assumed, without loss of generality, that it is in the $z$-direction of the coordinate system, and that $|\vec{B}| \ll\left|\vec{B}_{0}\right|$. 
Substituting equations $2.18,2.19$, and 2.20 into the equation of motion, eq. 2.14 , and keeping the terms of first order yields:

$$
-i \omega m_{\imath} \vec{v}_{\imath}=q_{0}\left(\vec{E}+\vec{v}_{\bullet} \times \vec{B}_{0}\right)
$$

This is a system of three equations in the three unknown components of the velocity, $\vec{v}$. When it is solved, this $3 \times 3$ system of equations for the components of $\vec{v}$, yields:

$$
\begin{aligned}
& v_{s x}=\frac{i q_{s}}{m_{s}\left(\omega^{2}-\omega_{s c}^{2}\right)}\left(\omega E_{x}+i \epsilon_{\lrcorner} \omega_{s c} E_{y}\right) \\
& v_{s y}=\frac{i q,}{m_{s}\left(\omega^{2}-\omega_{s c}^{2}\right)}\left(-i \epsilon_{,} \omega_{s c} E_{x}+\omega E_{y}\right) \\
& v_{e z}=\frac{i q_{0}}{m_{0} \omega} E_{z}
\end{aligned}
$$

where:

$$
\epsilon_{0}=\frac{q_{0}}{\left|q_{0}\right|}
$$

represent the sign of the charge and:

$$
\omega_{\text {oc }}=\frac{\left|q_{\triangleleft}\right| B_{0}}{m_{\triangleleft}}
$$

is the species cyclotron frequency of the plasma.

This solution can be seen as a matrix multiplied by a vector, that is:

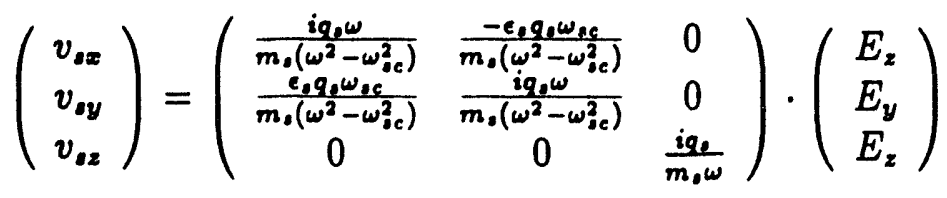

Now this matrix can be diagonalized by the rotation of the coordinate system or what is equivalent, by choosing a coordinate system such that the matrix becomes diagonal. This coordinate system is given by the transformations:

$$
\begin{aligned}
& v_{ \pm}=v_{x} \pm i v_{y} \\
& E_{ \pm}=E_{x} \pm i E_{y}
\end{aligned}
$$

which are obtained by the usual method of row multiplication and addition such that the matrix becomes diagonal. Substituting the values for the $v_{s x}$ and $v_{s y}$ from 
equations 2.22 and 2.23 into equation 2.28 , the components of the velocity in the diagonalized coordinate system become:

$$
v_{ \pm}=\frac{i q_{,}}{m_{\bullet}\left(\omega \mp \epsilon_{\text {o }} \omega_{\text {sc }}\right)} E_{ \pm}
$$

In a similar fashion, the current density can be expressed in vector form as:

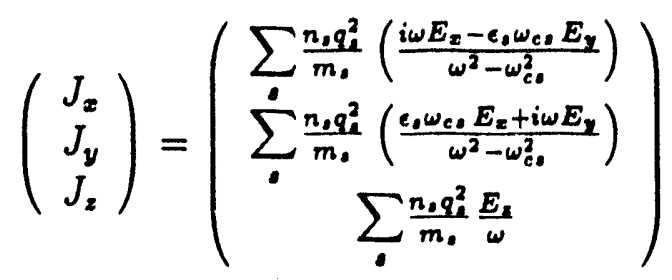

or in the diagonalized coordinate system as:

$$
\begin{aligned}
& J_{ \pm}=i \epsilon_{0} \sum_{s} \frac{\omega_{p s}^{2}}{\omega \mp \epsilon_{0} \omega_{\rho c}} E_{ \pm} \\
& J_{z}=i \epsilon_{0} \sum_{s} \frac{\omega_{p s}^{2}}{\omega} E_{z}
\end{aligned}
$$

where:

$$
\omega_{p s}^{2} \equiv \frac{n, q_{1}^{2}}{m_{,} \epsilon_{0}}
$$

is the species plasma frequency in the coordinate system of diagonalization.

Next consider Maxwell's equation 2.16, in particular the current terms on the right hand side of the equation. This equation can be used to define a new useful quantity, the dielectric tensor, $\overline{\overline{\mathrm{K}}}$, as:

$$
\vec{J}-i \omega \epsilon_{0} \vec{E}=-i \omega \epsilon_{0} \overrightarrow{\mathrm{K}} \cdot \vec{E}
$$

where the dielectric tensor $\bar{K}$ is given by:

$$
\overline{\overline{\mathrm{K}}} \equiv\left(\begin{array}{ccc}
S & -i D & 0 \\
i D & S & 0 \\
0 & 0 & P
\end{array}\right)
$$

where:

$$
S \equiv \frac{1}{2}(R+L) \equiv 1-\sum_{\bullet} \frac{\omega_{p \ell}^{2}}{\omega^{2}-\omega_{\jmath c}^{2}}
$$




$$
\begin{aligned}
D & \equiv \frac{1}{2}(R-L) \equiv \sum_{s} \frac{\epsilon_{s} \omega_{p s}^{2} \omega_{s c}}{\omega\left(\omega^{2}-\omega_{s c}^{2}\right)} \\
P & \equiv 1-\sum_{s} \frac{\omega_{p s}^{2}}{\omega^{2}} \\
R & \equiv S+D \equiv 1-\sum \frac{\omega_{p s}^{2}}{\omega\left(\omega+\epsilon_{s} \omega_{s c}\right)} \\
L & \equiv S-D \equiv 1-\sum_{s} \frac{\omega_{p s}^{2}}{\omega\left(\omega-\epsilon_{s} \omega_{s c}\right)}
\end{aligned}
$$

The notation used here is the familiar notation first used by $\mathrm{Stix}^{53}$ and are mnemonics for the words: Sum, Difference, Plasma, Right and Left.

The advantages of these definitions and formulae are that they makes an excellent launch path for the derivation of the dispersion relation for a wave in a plasma, which follows next.

Starting with Maxwell's equations 2.3 and 2.4, by taking the curl (i.e., $\nabla \times$ ) of equation 2.3 and 2.4 , it can be shown that: ${ }^{48,53}$

$$
\begin{aligned}
\vec{\nabla} \times(\vec{\nabla} \times \vec{E}) & =\vec{\nabla} \times\left(-\frac{\partial \vec{B}}{\partial t}\right) \\
\vec{\nabla} \times \vec{B} & =\mu_{0}\left(\vec{J}+\frac{\partial \overline{\bar{\epsilon}} \vec{E}}{\partial t}\right)
\end{aligned}
$$

which under the assumption that the fields are Fourier transformable, becomes:

$$
\begin{aligned}
\vec{k} \times(\vec{k} \times \vec{E}) & =\vec{k} \times \omega \vec{B} \\
\vec{k} \times \vec{B} & =-\omega \epsilon_{0} \mu_{0} \overline{\overline{\mathrm{K}}} \cdot \vec{E}
\end{aligned}
$$

Substituting equation 2.44 into equation 2.45 and defining the index of refraction vector as:

$$
\vec{n} \equiv \frac{\vec{k} c}{\omega}
$$

whose magnitude, $n=|\vec{n}|$, represents the ratio of the velocity of light in the medium to the phase velocity of the wave, yields:

$$
\vec{n} \times(\vec{n} \times \vec{E})+\overline{\mathrm{K}} \cdot \vec{E}=0
$$


where the identity:

$$
c^{2}=\frac{1}{\epsilon_{0} \mu_{0}}
$$

has been used.

Now, without loss of generality, assume the direction of propagation, $\vec{k}$, of the wave to be in the $x-z$ plane, and since $\vec{B}$ has been already chosen in the $z$-direction, equation 2.47 becomes:

$$
\left(\begin{array}{ccc}
S-n^{2} \cos ^{2} \theta & -i D & n^{2} \cos \theta \sin \theta \\
i D & S-n^{2} & 0 \\
n^{2} \cos \theta \sin \theta & 0 & P-n^{2} \sin ^{2} \theta
\end{array}\right)\left(\begin{array}{c}
E_{x} \\
E_{y} \\
E_{z}
\end{array}\right)=0
$$

where $\theta$ is the angle between the propagation vector $\vec{k}$ and the $z$-axis. This is the famous dispersion relationship of the "cold" plasma, and it describes the propagation modes of waves in the plasma. ${ }^{48,53}$

In order to have a nontrivial solution the determinant of the matrix must be zero. This condition gives the final form of the dispersion relation as:

$$
A n^{4}-B n^{2}+C=0
$$

where:

$$
\begin{aligned}
& A \equiv S \sin ^{2} \theta+P \cos ^{2} \theta \\
& B \equiv(S+D)(S-D) \sin ^{2} \theta+P S\left(1+\cos ^{2} \theta\right) \\
& C \equiv P(S+D)(S-D)=P R L
\end{aligned}
$$

The solutions can be written in terms of the angle as follows. Start by substituting equations $2.51,2.52$, and 2.53 into equation 2.50 to obtain:

$$
0=\left(S \sin ^{2} \theta+P \cos ^{2} \theta\right) n^{4}-\left[L R \sin ^{2} \theta+P S\left(1+\cos ^{2}\right)\right] n^{2}+P L R
$$

where the identity $L R=(S-D)(S+D)$ has been used. Next, use equation 2.54 twice. The first time express all the sines in terms of cosine using the formula 
$\sin ^{2} \theta=1-\cos ^{2} \theta$ and the second time express all the cosine in terms of the sines in order to obtain two equations, one in terms of cosine only and another one in terms of sines only. After moving the corresponding terms around in both equations:

$$
\begin{aligned}
S n^{4}-(L R+P S) n^{2}+P L R & =\cos ^{2} \theta\left[(P-S) n^{4}+(L R-P S) n^{2}\right] \\
P n^{4}-2 P S n^{2}+P L R & =\sin ^{2} \theta\left[(P-S) n^{4}+(L R-P S) n^{2}\right]
\end{aligned}
$$

Taking the ratio of equations 2.55 and 2.56 above, yields:

$$
\frac{\sin ^{2} \theta}{\cos ^{2} \theta}=\frac{P n^{4}-2 P S n^{2}+P L R}{S n^{4}-(L R+P S) n^{2}+P L R}
$$

The numerator and denominator can be factored into the form:

$$
\tan ^{2} \theta \equiv-\frac{P\left(n^{2}-R\right)\left(n^{2}-L\right)}{\left(S n^{2}-R L\right)\left(n^{2}-P\right)}
$$

where the general condition for resonance (where $n^{2} \rightarrow \infty$ or $\lambda \rightarrow 0$ ) is given by:

$$
\tan ^{2} \theta=-\frac{P}{S}
$$

and the general condition for cutoff (where $n^{2}=0$ or $\lambda \rightarrow \infty$ ) is given by:

$$
C=P(S+D)(S-D)=0
$$

There are two special cases for the propagation of a wave in a plasma. These are propagation parallel and perpendicular to the magnetic field. This paper deals only with propagation perpendicular to the magnetic field. For such waves there exist two modes of propagation:

1. $n^{2}=P$, known as the Ordinary mode or O-mode.

2. $n^{2}=\frac{R L}{S}$, known as the eXtraordinary mode or X-mode. 


\subsubsection{Properties of the $\mathrm{O}$ - and $\mathrm{X}$-modes}

This section presents some of the properties of the solution to a wave propagating perpendicular to the magnetic field of the plasma, the so called Ordinary and eXtraordinary waves. From here on, only the first order terms will be considered on the formulas and derivations.

The Ordinary mode The Ordinary wave follows the dispersion relation given by:

$$
n_{O}^{2}=P=1-\sum_{s} \frac{\omega_{p s}^{2}}{\omega^{2}}
$$

or when only the electrons are considered, in the simpler form:

$$
n_{O}^{2}=P=1-\frac{\omega_{p e}^{2}}{\omega^{2}}
$$

which shows the dependence of the wave on the local density, since $\omega_{p e}^{2}(r)=\frac{n_{e}(r) q_{e}^{2}}{m_{e} \varepsilon_{0}}$. The dispersion relation, equation 2.62, does not show any dependence on the magnetic field. This is because the electric field, $\vec{E}$, of the Ordinary wave is parallel to the magnetic field, $\vec{B}$, so that the particles (electrons) do not experience any effect from the magnetic field. There is no resonance (except for $\omega=0$, which does not count because it is basically D.C.) and the cutoff condition, $n^{2}=0$, occurs only at $\omega=\omega_{p c}\left(r_{c}\right)$, the electron plasma frequency. There can be no propagation (as discussed in section 2.6.2) for $\omega<\omega_{\text {pe }}$, since this makes $n^{2}<0$, and $n^{2} \rightarrow 1$ as $\boldsymbol{\omega} \rightarrow \infty$, making the $\mathrm{O}$-mode dispersion relation among the simplest and easiest to work with.

The propagation of the $\mathrm{O}$-mode is characterized by a direction of propagation, $\vec{k}$, perpendicular to the magnetic field, $\vec{B}_{0}$, and an electric field, $\vec{E}$, parallel to the externally applied magnetic field, $\vec{B}_{0}$. 
The eXtraordinary mode For the eXtraordinary wave the story becomes a little more complicated, with a dispersion relation given by:

$$
n_{X}^{2}=\frac{(S+D)(S-D)}{S}
$$

which can be express in terms of the electron frequencies as:

$$
n_{X}^{2}=1-\frac{\omega_{p e}^{2}}{\omega^{2}} \frac{\omega^{2}-\omega_{p e}^{2}}{\omega^{2}-\omega_{p e}^{2}-\omega_{e c}^{2}}
$$

The resonances, $n_{X}^{2} \rightarrow \infty$, for the $\mathrm{X}$-mode are given by the equations:

$$
\begin{aligned}
& \omega_{U H}^{2}=\omega_{p e}^{2}+\omega_{e c}^{2} \\
& \omega_{L H}^{2}=\omega_{e c} \omega_{i c}\left(\frac{\omega_{p e}^{2}+\omega_{e c} \omega_{i c}}{\omega_{p e}^{2}+\omega_{e c}^{2}}\right)
\end{aligned}
$$

where the subscripts refer to the Upper-Hybrid and Lower-Hybrid respectively, and some terms of the order $\frac{m_{e}}{m_{1}}$ have been neglected. The cutoff, $n^{2} \rightarrow 0$, occurs at:

$$
\omega_{X}= \pm \frac{\omega_{e c}}{2}+\left[\omega_{p e}^{2}+\left(\frac{\omega_{e c}}{2}\right)^{2}\right]^{\frac{1}{2}}
$$

where two negative roots have been discarded. The two cutoffs are referred to as the upper $(+)$ and the lower $(-)$ cutoffs, ${ }^{67}$ or as the right $(+)$ and left $(-)$ cutoffs, ${ }^{53,84}$ or as the fast $(+)$ and slow $(-)$ waves $^{48}$ (because of their phase velocities), respectively. Because of the number of different solutions and the many variables involved in plasma wave propagation problems, the solutions become extremely complicated. A popular way to sketch these solutions is the famous Clemmow-Mullaly-Allis diagram or $C M A$ diagram. ${ }^{48}$ In this type of diagram the cutoffs and resonances are plotted as curves as a function of the normalized density, $\frac{\omega_{p}^{2}}{\omega^{2}}$, and the normalized magnetic field, $\frac{\omega_{c a}}{\omega}$, for a one species plasma. An example is shown in Figure 2.1. ${ }^{48}$ The $x$ and $y$ axes are not linear. This is done to be able to include all of the boundaries in a clear fashion. A complete description of the CMA can be fund in the references, such as Budden, ${ }^{5}$ Ginzburg, ${ }^{8}$ Swanson, ${ }^{48}$ and others. ${ }^{51-55,133}$ 


\subsection{The CMA Diagram}

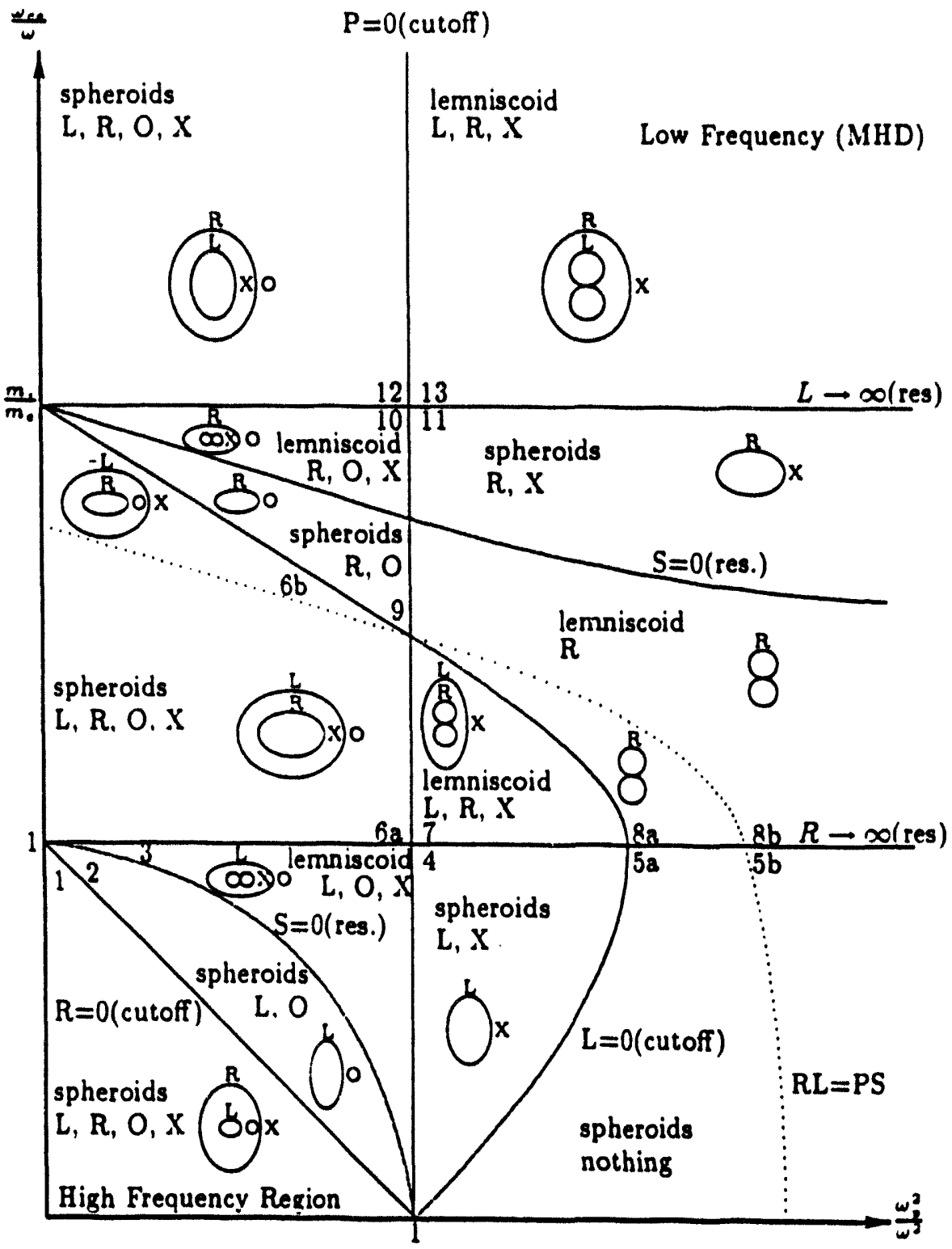

Figure 2.1: CMA diagram with all boundaries and the wave normal surfaces. 
For the propagation of the $\mathrm{X}$-mode the propagation vector, $\vec{k}$, is again perpendicular to the magnetic field, $\vec{B}_{0}$, but the electric field, $\vec{E}$, is perpendicular to the magnetic field, $\vec{B}_{0}$.

\subsubsection{Advantages and disadvantages of each mode}

O-mode The $\mathrm{O}$-mode has the advantage of having an extremely simple dispersion equation. Because of this, the formulae associated with the calculation of the density profile can be done analytically using an $A b e l$ inversion technique, as described in section 2.5.1. The problem with the Abel inversion is that it has an

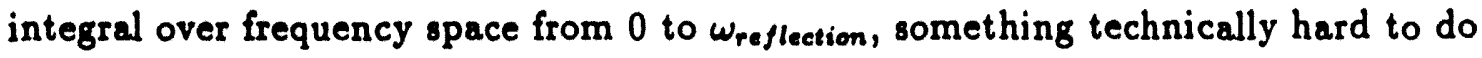
because there is no microwave generator that can go from $0 \mathrm{~Hz}$ to about $100 \mathrm{GHz}$ in a reasonable amount of time. The way that physicists have gone around this problem is by assuming a density profile at the edge and starting the frequency sweep at a more reasonable frequency. ${ }^{79,43}$ While this introduces some error in the technique, generally good agreement has been found between this and other diagnostic techniques. Because of the simplicity of the dispersion equation, it is the oldest technique in laboratory reflectometry and the one that people have the most experience.

A minor disadvantage of the $\mathrm{O}$-mode is that it cannot probe the back of the density profile on toroidal devices (where $B(R)=\frac{B_{0} R_{0}}{R}$ ), past the point of maximum density because of the single dependence of the dispersion relation on the density profile. Nor can it be used to probe the outer edge of the plasma, because the long wavelengths of the frequencies necessary to probe the plasma edge will reduce and degrade the spatial resolution of the beam.

$\mathrm{X}$-mode Because the $\mathrm{X}$-mode has a more complicated dispersion equation (compared with the one for the $\mathrm{O}$-mode), an analytical inversion is not pos- 
sible or known. However it has the advantage of being able to probe the edge of the plasma ${ }^{31}$ starting with the electron cyclotron frequency (equation 2.26) at that point, ${ }^{126}$ whereas the $\mathrm{O}$-mode has to use very low frequencies at such low densities. At the edge of the plasma, where $n_{e} \rightarrow 0$, the cutoff and the electron resonance layers become closer; thus, some of the waves may pass through the evanescent part of the plasma and be absorbed at the electron resonance layer. Regardless of this, the $\mathrm{X}$-mode wave makes it easier to probe the edge of the plasma (as compared to the O-mode). This advantage has been used in ASDEX (R. Schubert ${ }^{126}$ ) to study the density profile at the edge of the plasma in front of the RF-heating antenna.

Another advantage of the X-mode is that because of the dual dependance of the dispersion relation on the density and the magnetic field, the $\mathrm{X}$-mode wave can propagate past the point of maximum density and probe the back of the density profile (this is for toroidal devices, because $B(R) \propto \frac{1}{R}$ and as long as the density is sufficiently low). Some knowledge of the magnetic field profile is needed, but this is not a major obstacle because the usual assumption that $B(R) \propto \frac{1}{R}$ can be used. The scanning capabilities of the X-mode are restricted by the position of the second harmonic resonance layer and not by the peakedness of the density profile as compared with the $\mathrm{O}$-mode wave. This does not present a problem, since as can be seen in Figure 2.2, for a typical $n_{e} \propto\left[1-\left(\frac{e}{a}\right)^{\alpha}\right]^{\beta}$ density profile, the first frequency for the second harmonic resonance corresponds to the probing frequency for the innermost part of the density profile. This region is usually of less interest, because the radial profile is normally assumed to be symmetric in the minor radius. The density profile has been superimposed to show its effect on the cutoff frequencies (X-mode upper and lower cutoff) and on the Upper and Lower Hybrid resonance frequencies. Figure 2.2 shows some of the different resonances and cutoffs for an $\mathrm{X}$-mode wave; from the position of these resonances, it is clear that a ware in the frequency range of the reflectometer (90 to $120 \mathrm{GHz}$ ) launched from the low 
field side of the plasma will always reach the cutoff before it reaches the Upper Hybrid and/or Electron Cyclotron resonances. The toroidal field coil current, $I_{t f}$, the corresponding magnetic field, $B_{0}$, the central density, $n_{\mathbb{a}}(0)$, and coefficients for the assumed density profile ( $\alpha$ and $\beta$ ) are shown in the figure. The density profile is plotted to show the general shape of a typical plasma and to show the effect of the density profile on the plasma frequencies.

\subsubsection{Solution to the wave equation in a plasma}

The wave equation has the general form:

$$
\vec{\nabla}^{2} \vec{E}(\vec{r}, t)+\frac{1}{c^{2}} \frac{\partial^{2} \vec{E}(\vec{r}, t)}{\partial t^{2}}=0
$$

where the general electric vector field has a dependance on both the position, $\vec{r}$, and the time, $t$. Solution of this equation at any point and time is rather difficult. So, as usual, different approximations for different conditions and regions of the plasma have been found. The importaice of finding such solutions for reflectometry is that the phase of the reflecting wave can be obtained from such solutions, and from the phase, the density profile can be reconstructed. Some of these approximations and solutions follow.

Geometrical optics solution The geometric optical appoximation can be used to calculate the phase for a wave propagating in a fusion plasma given that some assumptions about the plasma medium are made. This assumptions are:84

1. The effects of the toroidal curvature will be ignored. This is a valid assumption, since the wave is launched along the minor radius and the beam width is much smaller than the major radius of the device. Any waves not propagating in the direction of the major radius will be reflected away from the collecting antenna and are very unlikely to be detected. 
$I=67.00 \mathrm{kAmpS} \quad \mathrm{ALPHA}=6.00 \mathrm{BETA}=2.00$

21-JUL-93

$B=4.50$ DENSO $=4.50 E+19 \mathrm{PART} / \mathrm{m}^{-3}$

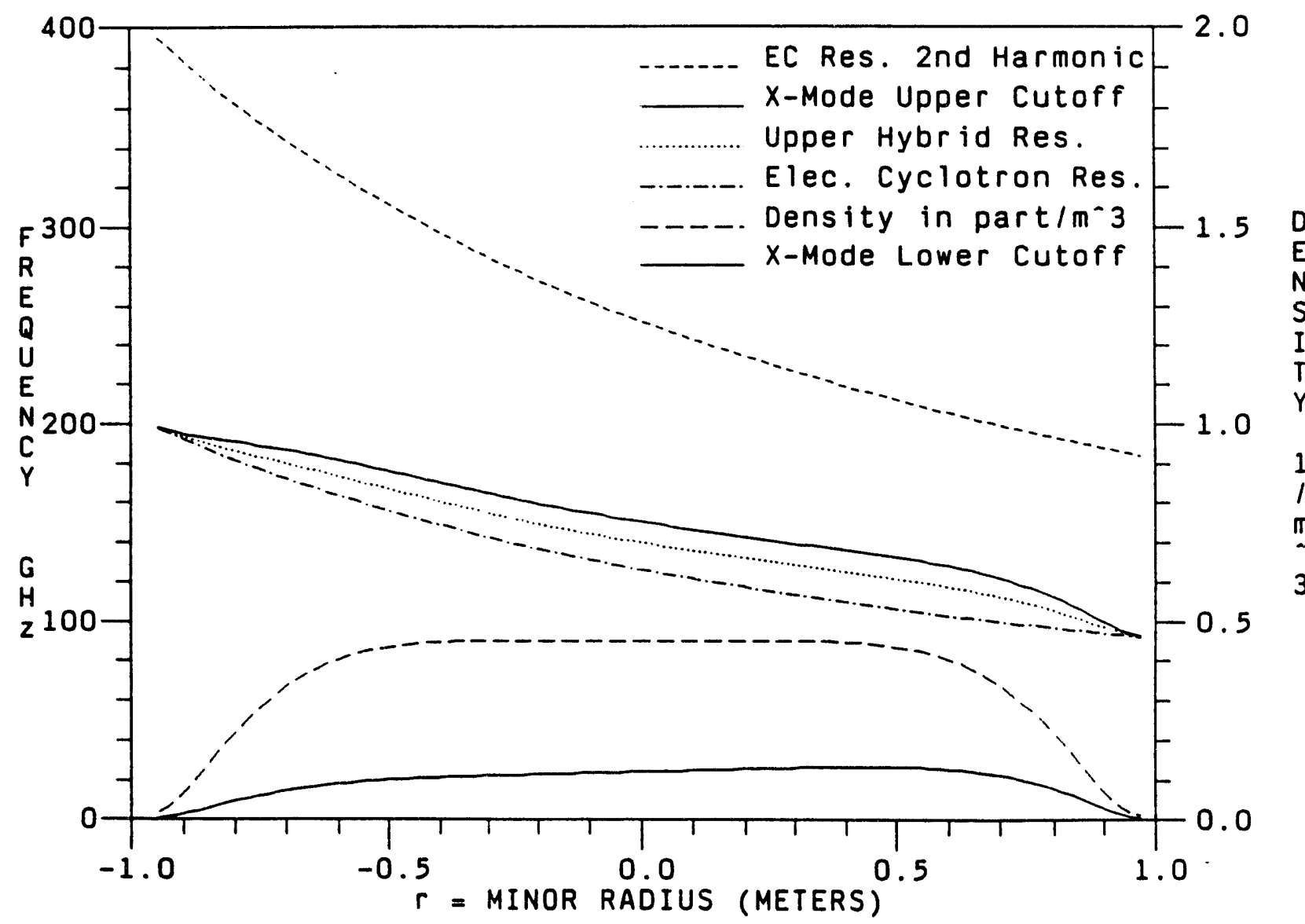

Figure 2.2: Plot of the different frequencies for a plasma with a density profile of the type $n_{e}(r) \propto\left(1-\left(\frac{r}{a}\right)^{\alpha}\right)^{\beta}$ and with a magnetic field (in Teslas) of the form $B(R) \propto \frac{1}{R}$, where $r$ is the minor radius, $a$ is the plasma edge minor radius and $R$ is the major radius. DENSO is the center density. 
2. The electron density, $n_{e}$, has only a radial dependence. As long as the data is collected in about $200 \mu$-seconds the average density will be time independent.

3. The magnetic field, $\vec{B}$, has only a radial dependence too. Here again, the effects of the toroidal curvature are ignored and a time independent magnetic field is assumed for the time scales of the collection of the data.

4. The fields are Fourier transformable in time.

5. Absorption is neglected.

On the basis of the above assumptions, equation 2.68 becomes:

$$
\frac{d^{2} \vec{E}(\vec{r})}{d r^{2}}+\frac{\omega^{2}}{c^{2}} \epsilon_{r}(\vec{r}, \omega) \vec{E}(\vec{r})=0
$$

where $\epsilon_{r}(\vec{r}, \omega)=n^{2}$ is the relative permittivity and $n$ is the index of refraction.

For a $100 \%$ homogeneous plasma, i.e., for $\epsilon_{r}=n^{2}=$ constant, the solution becomes:

$$
E(r)=E_{0} e^{ \pm i \frac{\omega}{c} n r}
$$

where $E_{0}$ is simply a constant and the phase is given by:

$$
\phi=\frac{\omega n r}{c}
$$

To add more reality to the problem, consider $\epsilon_{r}(r)$ as a slowly changing function of $r$; in such a case, a reasonable approximation is to substitute the term $n r$ in equation 2.71 by the average refraction coefficient over the ray path. Then the phase will become:

$$
\phi=\frac{\omega}{c} \int_{r_{1}}^{r_{2}} n(r, \omega) d r
$$

where $r_{1}, r_{2}$ are the end points of the path along the minor radius of the plasma.

This by itself is the solution of the geometrical optics approximation, or the WKB or WKBJ approximation, first introduced by Jeffreys ${ }^{87}$ and developed 
for quantum mechanics and the Schrödinger equation by Wentzel, ${ }^{88} \mathrm{Kramers},{ }^{89}$ and Brillouin. ${ }^{90}$ It holds only when $\epsilon_{r}(r)$ changes slowly over the length of an electromagnetic wave, that is, when:

$$
\lambda(r) \frac{d \epsilon_{r}(r)}{d r} \ll \epsilon_{r}(r)
$$

for which $\lambda(r)$ is the wavelength at position $r$. This condition is equivalent to:

$$
\frac{\lambda}{L_{n_{e}}} \ll 1
$$

for the O-mode and to:

$$
\frac{\lambda}{L_{n_{e}}}, \frac{\lambda}{L_{B}} \ll 1
$$

for the X-mode, where $L_{n_{e}}$ is the electron density gradient scale length:

$$
L_{n_{e}}=n_{e}\left(\frac{d n_{e}}{d r}\right)^{-1}
$$

and $L_{B}$ is the magnetic field gradient scale length:

$$
L_{B}=B\left(\frac{d B}{d r}\right)^{-1}
$$

Series solution Others have obtained solutions by assuming other forms for the solution. Ginzburg ${ }^{8}$ seeks a solution by assuming a solution in the form of a series, as:

$$
E(r)=\sum_{j=0}^{\infty}\left(\frac{c}{\omega}\right)^{j} E_{j}(r) e^{ \pm i \frac{\omega}{c} \psi(r)}
$$

where $\psi(r)$ and $E_{j}(r)$ are unknown functions. Substituting equation 2.78 into equation 2.69, collecting terms of equal powers in $\frac{c}{\omega}$ and setting the terms of each of the powers of $\frac{c}{\omega}$ to 0 , yields:

$$
\begin{aligned}
\psi(r) & = \pm \int_{r_{1}}^{r} n\left(r^{\prime}, \omega\right) d r^{\prime} \\
E_{0}(r) & =A_{0}(n(r, \omega))^{-\frac{1}{2}} \\
E_{1}(r) & =\left.(n(r, \omega))^{-\frac{1}{2}} \int_{r_{1}}^{r} \frac{1}{2 i}\left(\frac{d^{2} E_{0}(x)}{d x^{2}}\right)\right|_{x=r^{\prime}}\left(n\left(r^{\prime}, \omega\right)\right)^{-\frac{1}{2}} d r^{\prime}
\end{aligned}
$$


Assuming that only the first term of the series is significant, the solution is:

$$
E(r)=A_{0}(n(r, \omega))^{-\frac{1}{2}} e^{ \pm i \frac{\psi}{e} \int_{r_{1}}^{r} n\left(r^{\prime}, \omega\right) d r^{\prime}}
$$

which is very similar to the solution for constant $n(r, \omega)$ and even more interesting is that the phase solution is exactly the same.

If $n(r, \omega)$ is monotonic, a sufficient condition for the validity of equation 2.83 is that:

$$
\frac{\omega}{c} \frac{n^{2}}{\left|\frac{d n}{d r}\right|}=\frac{2 \pi L_{n}}{\lambda} \gg 1
$$

where $L_{n} \equiv n^{2}\left(\frac{d n}{d r}\right)^{-1}$ is the gradient scale length of the refractive index, $n$. Physically, the above equation means that the local wavelength of the probing wave must be much smaller than the local gradient scale length of the refractive index.

Solution at the reflection layer An exact solution for the wave equation in the neighborhood of the reflecting layer has been calculated for an electromagnetic wave moving in the ionosphere. ${ }^{5,8}$ The assumptions to reach this solution are:

1. The plasma electron density is monotonically increasing with decreasing minor radius.

2. The plasma is collisionless.

3. The density, $n_{\mathrm{e}}(r)$, is approximately linear around the neighborhood of the reflection layer. In his derivation, Budden ${ }^{5}$ shows that a sufficient condition for $n_{e}(r)$ to be considered quasi-linear is that:

$$
\frac{d^{2} n_{e}(r)}{d r^{2}}\left|r-r_{c}\right| \ll \frac{d n_{e}}{d r}
$$

where $r_{c}$ is the position where the wave is reflected or the cutoff position. 
In this region of quasi-linearity the relative permittivity of the plasma can be expressed as:

$$
\epsilon_{r}=a_{0}\left(r-r_{c}\right)
$$

where $a_{0}$ is a constant. Under this condition the wave equation becomes:

$$
\frac{d^{2} \vec{E}(\vec{r})}{d r^{2}}+\frac{\omega^{2}}{c^{2}} a_{0}\left(r-r_{c}\right) \vec{E}(\vec{r})=0
$$

This happens to be one of Stokes' differential equations with solutions in terms of the famous Airy integrals. ${ }^{139}$ In his derivation, Budden ${ }^{5}$ shows that the solution at the reflection layer matches the solution of the geometrical optics approximation with a difference of a term of $\frac{\pi}{2}$ due to the reflection of the wave. The final solution for the phase is:

$$
\phi=2 \frac{\omega}{c} \int_{r_{1}}^{r_{2}} n(r, \omega) d r-\frac{\pi}{2}
$$

in which the factor of 2 comes from assuming a symmetrical path of the incident and reflected wave to and from the reflecting layer.

One dimensional full wave analysis. A one dimensional full wave solution to the wave equation of a reflectometer beam has been done and studied by Hutchinson. ${ }^{34}$ The analysis is based on the first Born approximation (which is not a full wave solution, but another type of solution), dating back at least to Pitteway (1959). ${ }^{36}$ Even though it is a one dimensional approach, Hutchinson's solution includes some features (such as, wave attenuation due to beam divergence and/or absorption by the medium) that correspond to multidimensional analysis of the wave. This is done by considering the case where the wave number, $k$, and the solution to the wave equation are complex. This analysis differs from the more usual WKBJ approximation ${ }^{5,8}$ in offering a solution consistent with the full wave problem but requiring that the perturbations to the density profile are small. In comparison, the WKBJ approximation ignores the finite wavelength but can be applied to zero-order problems, and hence to large perturbations, at least in principle. 
The assumptions for this model are:

1. Consider a plasma slab in which all gradients are perpendicular to the magnetic field.

2. Without loss of generality, assume that the magnetic field, $\vec{B}$, is in the $\hat{z}$ direction and the wave propagates in a direction parallel to the gradients, which it is taken in the $\hat{x}$-direction.

3. Next, assume that the plasma can be described by a local response in the form of a dielectric tensor, $\overline{\bar{\epsilon}}$, that is a function only of the frequency, $w$, and the position, $x$, such that the wave equation is:

$$
\vec{\nabla} \times(\vec{\nabla} \times \vec{E})-\frac{\omega^{2}}{c^{2}} \overline{\bar{\epsilon}} \cdot \vec{E}=0
$$

4. Finally, assume that the cross terms $\epsilon_{x z}$ and $\epsilon_{y z}$ are zero.

Under these assumptions, equation 2.89 can be separated into the two decoupled scalar wave equations:

$$
\left[\frac{d^{2}}{d x^{2}}+\frac{\omega^{2}}{c^{2}}\right] E_{z}=0
$$

for the O-mode, and:

$$
\left[\frac{d^{2}}{d x^{2}}+\frac{\omega^{2}}{c^{2}}\left(\epsilon_{y y}-\frac{\epsilon_{y x} \epsilon_{x y}}{\epsilon_{x x}}\right)\right] E_{y}=0
$$

for the X-mode, where the values for the components of the dielectric tensor are given by:

$$
\begin{aligned}
\epsilon_{x x} & =1-\frac{\omega_{p e}^{2}}{\omega^{2}-\omega_{e c}^{2}} \\
\epsilon_{y y} & =\epsilon_{x x} \\
\epsilon_{x y} & =i \frac{\omega_{p e}^{2} \omega_{e c}}{\omega\left(\omega^{2}-\omega_{e c}^{2}\right)} \\
\epsilon_{y x} & =-\epsilon_{x y} \\
\epsilon_{x z} & =\frac{\omega_{p e}^{2}}{\omega^{2}}
\end{aligned}
$$


Both of equations 2.90 and 2.91 are of the Helmholtz type, that is, of the form:

$$
\frac{d^{2} \psi}{d x^{2}}+k^{2}(x) \psi=0
$$

where the parameter $k(x)$ is an arbitrary fuaction of $x$. In the particular case of the O-mode. $k$ is given by:

$$
\begin{aligned}
k^{2}(x) & =\frac{\omega^{2}-\omega_{j=1}^{2}(x)}{c^{2}} \\
& =k_{v}^{2}\left(1-\frac{n_{c}(x)}{n_{\mu}\left(x_{t}\right)}\right)
\end{aligned}
$$

where $k_{2}=\frac{n}{6}$ and $n_{1}\left(x_{c}\right)$ is the critical density, or the densily al the cutoff, given by:

$$
n_{c}\left(x_{i}\right)=\frac{\dot{\nu}^{2} c_{0} \pi l_{0}}{q^{2}}
$$

and $n_{0}(x)$ is the local densiry. While for the $X$-mode, $k$ is given by:

$$
k^{2}(x)=k_{v}^{2}\left[1-\frac{\omega_{p e}^{2}(x)\left(\omega^{2}-\omega_{p e}^{2}(x)\right)}{\omega^{2}\left(\omega^{2}-\omega_{e c}^{2}(x)-\omega_{p e}^{2}(x)\right)}\right]
$$

Equation 2.97 is a second order linear differcntial equation with two linearly independent solutions, call them $i_{1}$ and $i_{2}$. The one that represents the physical solution has to go to zero as $x \rightarrow \infty$. Without loss of generality; it can be assume that $\psi_{1}$ is such a solution. A perturbation on the amplitude of the density (like the fluctuations due to $\mathrm{MHD}$ and other instabilities) will induce a similar perturbation on the wave number. $k$, because as it can be seen from equations 2.98 and 2.101 above:

$$
\begin{aligned}
k & =k\left(u_{p c}\right) \\
& =k\left(u_{p c}\left(n_{e}\right)\right)
\end{aligned}
$$

for both the $O$-mode and the X.mode. So, by adding a small perturbation to the $k$ due to thr. perturbation on the density. the new solution to equation 2.97 can be 
and

$$
\psi \rightarrow \psi_{0}+\bar{\psi}
$$

where the symbol - stands for the perturbed part of the new solution. Then equation 2.97 becomes:

$$
\frac{d^{2} \tilde{\psi}}{d x^{2}}+k_{0}^{2} \tilde{\psi}=-\dot{k}^{2} \psi_{0}
$$

which still is in the form of Helmholtz equations and its solution can be found using the ubiquitous Green functions. Green's theory says that given an equation of the form:

$$
\frac{d^{2} \psi}{d x^{2}}+k^{2} \psi=f(x)
$$

with boundary conditions:

$$
\begin{gathered}
\psi(x \rightarrow \infty)=0 \\
U\left(\psi\left(x_{0}\right)\right)=0
\end{gathered}
$$

where $U(\psi)$ is a linear combination operator of $\psi$ and $\frac{d \psi}{d x}$, evaluated at some reference point, $x_{0}$, inside of the vacuum region, then the solution to the boundary value problem is given by:

$$
\psi(x)=\int_{x_{0}}^{\infty} G(x, y) f(y) d y
$$

where $G(x, y)$ is the solution to the equation:

$$
\frac{d^{2} G(x, y)}{d x^{2}}+k^{2} G(x, y)=\delta(x-y)
$$

and $G(x, y)$ is given by:

$$
\begin{aligned}
G(x, y) & =\psi_{1}(y)\left(\psi_{2}(x)-\frac{U\left(\psi_{2}\left(x_{0}\right)\right)}{U\left(\psi_{1}\left(x_{0}\right)\right)} \psi_{2}(x)\right) \frac{1}{W(y)}, \text { for } x<y \\
& =\psi_{1}(x)\left(\psi_{2}(y)-\frac{U\left(\psi_{2}\left(x_{0}\right)\right)}{U\left(\psi_{1}\left(x_{0}\right)\right)} \psi_{2}(y)\right) \frac{1}{W(y)}, \text { for } y<x
\end{aligned}
$$

where $W(x)$ is the Wronskian, given by:

$$
W(x)=\psi_{1}(x) \psi_{2}^{\prime}(x)-\psi_{2}(x) \psi_{1}^{\prime}(x)
$$


where the primes symbol, ', denotes differentiation with respect to the argument of the function. The Green's function solution for the perturbation problem will be good provided that $\bar{\psi} \ll \psi_{0}$ for all $x$. Setting the zeroth solution to be:

$$
\psi_{0}=\psi_{1}
$$

and setting:

$$
f(x)=-\overline{k^{2}} \psi_{1}
$$

on equation 2.106, together with the boundary condition that equation 2.109 is evaluated at a point on the vacuum region, then the solution for the perturbed part of the solution for the wave equation is of the form:

$$
\bar{\psi}(x)=-\left(\psi_{2}(x)-\frac{U\left(\psi_{2}\right)}{U\left(\psi_{1}\right)} \psi_{1}(x)\right) \int_{\infty_{0}}^{\infty} \tilde{k^{2}}(\xi) \frac{\left(\psi_{1}(\xi)\right)^{2}}{W(\xi)} d \xi
$$

Thus, the first order perturbation solution to the wave equation using Green's functions is a linear combination of the two independent solutions to the unperturbed problem, with coefficients given by equation 2.116 .

In his work, Hutchinson ${ }^{34}$ proposes that the key principal effects of transverse wave spreading can reasonably be modelled by the addition of an imaginary part to $k^{2}$. This imaginary part is regarded as a parameter that can be adjusted to model approximately the effect of a specific antenna configuration.

Continuing with the derivation, it is assumed that in the vacuum region, where $k_{v}$ is constant, the unperturbed solution, $\psi_{1}$, can be decomposed into forward and backward propagating waves; that is:

$$
\psi_{1}=A_{1} e^{i k_{v}\left(x-x_{0}\right)}+B_{1} e^{-i k_{*}\left(x-x_{0}\right)}
$$

where the time dependent factor $e^{-i \omega t}$ is understood and $A_{1}$ and $B_{1}$ are the complex amplitudes of the forward and backward waves. Then at the reference point position, 
$x_{0}$, the amplitudes of the wave are:

$$
\begin{aligned}
& A_{1}=\frac{1}{2}\left(\psi_{1}-\frac{i \psi_{1}^{\prime}}{k_{v}}\right) \\
& B_{1}=\frac{1}{2}\left(\psi_{1}+\frac{i \psi_{1}^{\prime}}{k_{v}}\right)
\end{aligned}
$$

The second solution, $\psi_{2}$, can be expressed in a similar form, that is, with amplitudes $A_{2}$ and $B_{2}$ which are free to choose as needed, as long as the result is linearly independent of the solution $\psi_{1}$. Thus, the amplitudes for the second solution, $\psi_{2}$, can be:

$$
\begin{aligned}
& A_{2}=-i A_{1} \\
& B_{2}=i B_{1}
\end{aligned}
$$

For such solutions the Wronskian at the point $x=x_{0}$ is:

$$
\begin{aligned}
W\left(x_{0}\right) & =\left(A_{1}+B_{1}\right) i k_{v}\left(A_{2}-B_{2}\right)-\left(A_{2}+B_{2}\right) i k_{v}\left(A_{1}-B_{1}\right) \\
& =4 k_{v} A_{1} B_{1} \\
& =k_{v}\left(\psi_{1}^{2}+\frac{\psi_{1}^{\prime 2}}{k_{v}^{2}}\right)
\end{aligned}
$$

From general Wronskian theory ${ }^{35}$ it is known that for an equation of the form of equation 2.97, which has no first order term, the Wronskian, equation 2.113 , is a constant independent of $x$. Thus, for the present case, the value of $W(x)$ given by equation 2.122 at the point $x_{0}$ is the same for all $x$ in the plasma.

Under the assumption that the physical situation to be described is one in which the launched (forward) wave amplitude is determined by the apparatus used and is fixed, then the boundary condition to be applied to the forward amplitude of the perturbation should be zero. This will require the condition:

$$
U(\psi)=\left.\frac{1}{2}\left(\psi-\frac{i}{k_{v}} \psi^{\prime}\right)\right|_{\Sigma_{0}}
$$


From this choice of amplitude and boundary conditions it can be obtained:

$$
\frac{U\left(\psi_{2}\right)}{U\left(\psi_{1}\right)}=\frac{A_{2}}{A_{1}}
$$

and consequently the perturbation gives a forward and backward amplitudes of:

$$
\ddot{A}=0
$$

and:

$$
\begin{aligned}
\dot{B} & =-\left(B_{2}-B_{1} \frac{A_{2}}{A_{1}}\right) \int \overline{k^{2}} \frac{\psi_{1}^{2}}{W} d x \\
& =-2 i B_{1} \int \bar{k}^{2} \frac{\psi_{1}^{2}}{W} d x
\end{aligned}
$$

which is the general form of the perturbation solution and its importance is that from it the phase difference of the reflected wave can be obtained. The phase can be calculated from equation 2.128 for the total reflected wave, $B_{1}+\dot{B}$, whose phase angle relative to $B_{1}$ is:

$$
\dot{\phi}=-2 \Re\left(\int \dot{k}^{2} \frac{\psi_{1}^{2}}{W} d x\right)
$$

where the perturbation $\tilde{\phi}$ is small and $\Re(f)$ is the real part operator of $f$. Thus the perturbation on the phase of a wave can be calculated from the perturbation on the $k\left(n_{e}\right)$ of the wave. This approach to calculate the phase of the wave can be used when the perturbations to the density profile (and the $k$ ) are small, but the effect of these perturbations on the phase are not neglegible.

This section presented a derivation to calculate the phase changes due to the effects of density fluctuations on the traveling wave as it propagates through the plasma. The solution can be used to study fluctuations in the phase of the wave due to such effects as line density ripple interference and Bragg effect from density fluctuations. This solution provides a relatively simple one-dimensional full wave analysis that includes some effects (like beam dispersion and absorption by the plasma) which are characteristic of multidimesional analysis, without the usual complications that acompany multidimensional analysis derivations. 


\subsection{Reflectometry Techniques}

Reflectometry techniques have the advantages of requiring little access to the plasma, being non disturbing to the plasma, can probe the edge as well as the center of the plasma and providing density fluctuation information and/or density profile information, depending on the mode of operation and the technique being used. Each of these techniques has its advantages and disadvantages. A description of these follows.

\subsubsection{Fixed frequency}

Fixed frequency reflectometry has been used to provide information on the inception of a cutoff layer (that is, when a particular density or the peak density is reached some where in the plasma $)^{17,18,38,78}$; for movements and/or fluctuations of the cutoff layer ${ }^{23,30,128}$ (fluctuations in the density); for drift waves and transport studies, ${ }^{27,30,80,81,98,101,104-100,117}$ and for correlation fluctuations studies in which the signals from two or more independent sources are correlated ${ }^{103,84-88}$ to obtain information on radial coherence. ${ }^{91,118-120}$ In principle, these fixed frequency techniques are simple, compared with broad band swept frequency techniques, since there is only one frequency to observe and availability of components and matching is easy and reliable. The first experiments using fixed frequency reflectometry were done by Anisimov ${ }^{17}$ and Anoshkin ${ }^{18}$ in the Alpha device in the 1960s.

After Anoshkin's experiments, fixed frequency reflectometry for density fluctuations became extremely popular. Other diagnostics can provide density fluctuation information, but they are confined to the edge and disturb the plasma around them (like Langmuir probes), or are more complicated in design, are expensive, and require more access to the plasma, such as the Heavy Ion Beam Probe (HIBP), or may have poor spatial resolution (e.g., Far Infrared scattering because of the large 
scattering volume).

Two or more probing beam frequencies in the same antenna system can be used to do fluctuation correlation studies, in which the fluctuations at the radial reflection points of the corresponding probing beams can be cross correlated to obtain information such as the correlation length, normalized coherence, cross power spectral intensity (CPSI), cross phase of the fluctuation, and the radial width of a coherent fluctuation. ${ }^{84,85}$

\subsubsection{Narrow band swept frequency}

Narrow band or FM reflectometers have been used to measure the approximate position and movement of the cutoff layer, ${ }^{77,23}$ to determine the inception of the cutoff density (as in $\mathrm{PLT}^{7 \theta}$ ), to control auxiliary high power plasma heating systems and to infer density profiles if multiple sources are used. ${ }^{92,83,46,46}$ They are very much like narrow band FM radar as described by Doane ${ }^{79}$ and Lehecka. ${ }^{92,93}$ The idea of using multiple sources to calculate the density profile of a plasma was first mentioned by A. I. Anisimov ${ }^{17}$ in 1961 , and it is used in tokamaks like RTP. ${ }^{24}$

\subsubsection{Broadband swept frequency}

Broadband reflectometry was first proposed by Cano and Cavallo 140 in 1980. They proposed the determination of the density profile of a tokamak plasma by sweeping the probing frequency beam over the whole bandwidth of the waveguide and calculating the density profile out of the recorded phase of the reflected wave. After that, the method was tested by F. Simonet ${ }^{21,22}$ on the TFR tokamak using the 0 -mode of propagation and by others in different experiments, like Petula$B,{ }^{43,44}$ DIII-D, ${ }^{92-97}$ TF-1, ${ }^{67}$ JET, ${ }^{69}$ Wendelstein VII-AS, ${ }^{31,121}$ RFX, ${ }^{122}$ ASDEX, ${ }^{124-127}$ TORE-SUPRA ${ }^{129}$ and others. 
The method was able to provide very detailed profiles, but it was apparent that determining the complete density profile for a large tokamak plasma would require a large number of reflectometers working at adjacent frequency bands. This appeared to be an inherent problem with the 0 -mode propagation because to scan a plasma from an edge density of 0 to a density of $n_{0}$ the frequency band has to be from $0 \mathrm{~Hz}$ to $\omega_{0}$. On a fusion plasma with $\omega_{0}=50 \mathrm{GHz}$, it puts an impossible demand on the swept wave oscillator and the waveguide, since there is no oscillator with a frequency band from 0 to $50 \mathrm{GHz}$ and no waveguide that works over that entire frequency band. Sweeping from some $\omega_{\min } \rightarrow \omega_{0}$ has been done, but the error introduced by the missing information can be significant. This is an advantage of the $\mathrm{X}$-mode over the $\mathrm{O}$-mode, since to probe the plasma from the edge at density 0 to some point inside the plasma at density $n_{0}$ the $\mathrm{X}$-mode wave frequency goes from the electron cyclotron frequency (some $50 \mathrm{GHz}$ for a typical plasma) to the frequency of the cutoff. This makes it possible in principle to reduce the number of waveguides penetrating the vacuum vessel to one or two. The problem with the $\mathrm{X}$-mode is that its index of refraction depends on the total magnetic field as well as the density profile, so a reasonable knowledge or model of the magnetic field in the region of propagation must be available.

The profile inversion is among the important differences between the $\mathrm{O}$-mode and the $\mathrm{X}$-mode. For the $\mathrm{O}$-mode the density profile can be obtained by an Abel inversion of the data. This is possible because of the relatively simple form of the index of refraction and the single dependence on the density. For the X-mode, the index of refraction is a complicated function of the density and the local magnetic field. So an analytical solution as in the case of the Abel inversion for the O-mode is not known, and a numerical solution is necessary. Numerical algorithms have been developed by H. Bottollier et al..$^{43}$ and by E. J. Doyle. ${ }^{94,97}$ Bottollier's method has some numerical instability problems during the profile inversion. Doyle's method is 
similar to Bottollier's but it does not suffer from the numerical instability problems encountered by Bottollier.

\subsubsection{AM technique}

On the Amplitude Modulated (AM) reflectometer the amplitude of the probing signal (in the range of $\mathrm{GHz}$ ) is modulated (with a sinusoidal envelope) at a frequency on the order of $\mathrm{MHz}$ before launching it to the plasma. The advantage of this method is that phase tracking of the wave can be done at the modulation frequency of the sinusoidal envelope, reducing the phase shift. The group delay time, $\tau$, of the reflected signal can be easily evaluated from the phase delay, $\Delta \phi$, as:

$$
\tau=\frac{\partial \phi}{\partial \omega}=\frac{\Delta \phi}{\Omega}
$$

where $\Omega$ is the modulation frequency of the probing wave. A generic block diagram of a typical AM (sinusoidal modulation) reflectometer ${ }^{28,32}$ system is shown in Figure 2.3. This technique has been used in machines like $P B X-M^{33}$ to measure the edge density profile. The schematics for the AM reflectometer used in PBX-M is shown in Figure 2.4. Note the simplicity of the diagram as compared to the dual frequency phase difference reflectometer in Figure 4.1.

Some of the problems with an AM reflectometer are fast density fluctuations, pulse/envelope deformation, and parasitic reflections. The fast density fluctuations affect the signal by phase and amplitude oscillations. This effect can be significantly reduced provided that the modulation frequency is larger than the frequencies of the amplitude oscillations of the reflected signal. Pulse deformation is due to the response of the square law detector to higher order derivatives of the phase delay versus frequency and spectra deformation by dispersive effects of the waveguides and plasma. This presents a problems for pulse reflectometry (section 2.3.6), because of the broad spectrum of the square pulse. Since the AM (sinusoidal modulation) signal 


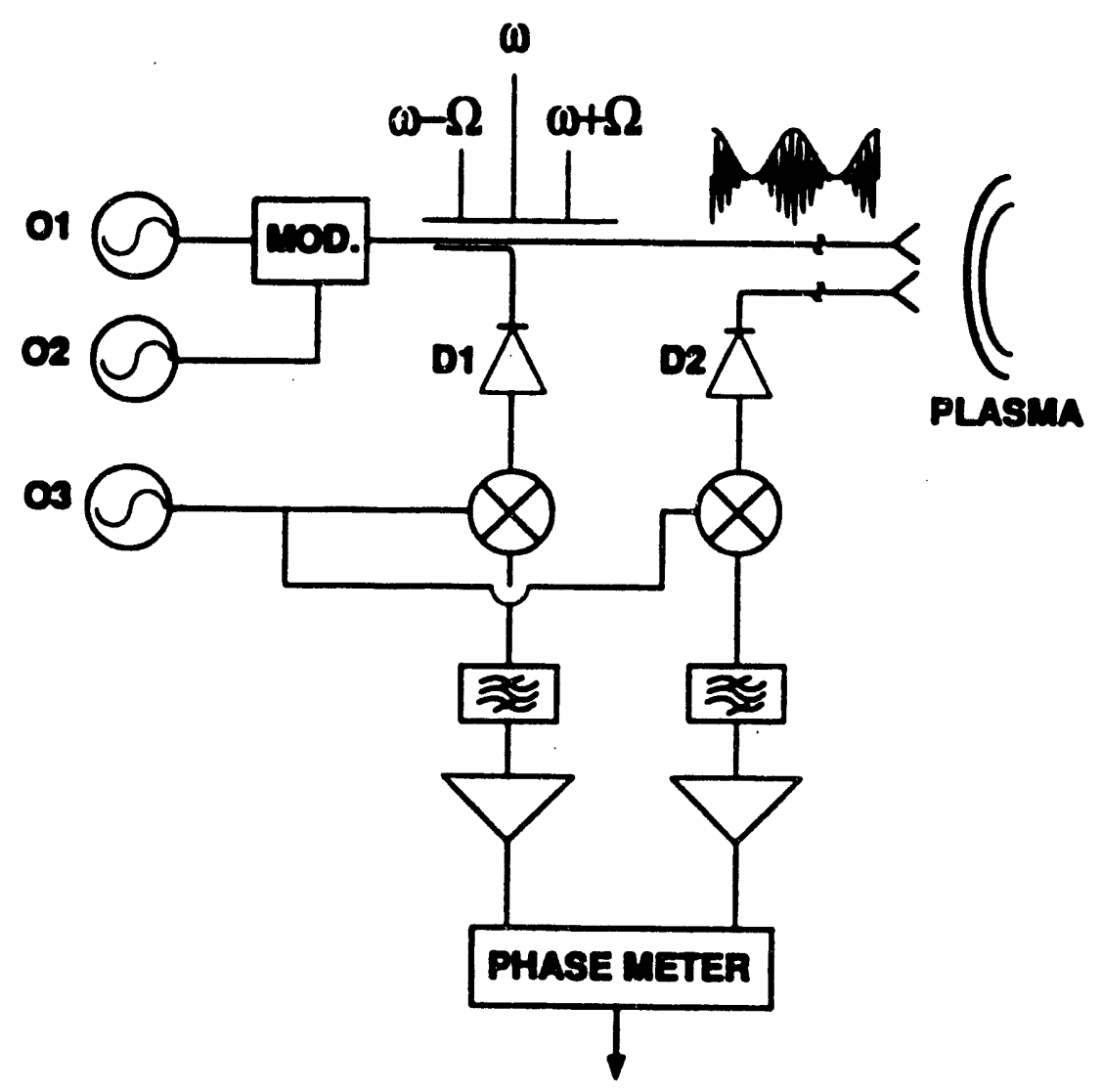

Figure 2.3: Generic diagram for an AM reflectometer system. 
is made up of only three frequency components, the deformation due to dispersion of the waveguide and plasma is minimal, and it can be totally suppressed by removing the carrier frequency or one of the side bands. ${ }^{28,32}$

\subsubsection{Dual frequency phase difference technique}

The dual frequency phase difference technique uses the difference in phase between two probing frequencies that are separated by a constant $\delta \omega$ and a technique similar to the one used by Doyle ${ }^{94,97}$ to reconstruct the density profile by adding the pieces of phase differences as the density profile is reconstructed. Since this technique uses the phase difference between the side bands, the fringe count is smaller than it is with the full phase detection technique. With the continuous full phase detection scheme a count of over 1000 fringes is observed, and keeping track is extremely difficult. With the dual frequency phase difference, the fringe count is on the order of 5 fringes, an easy task for the hardware..$^{28,39,40}$ More details are presented in section 2.5.2.

\subsubsection{Pulse radar technique}

A radar pulse of microwaves can be used to determine the density profile by either calculating the time of flight of the pulse (as is done in ionospheric studies ${ }^{5,6,8}$ ) or by using the change in shape of the reflected pulse. The time of flight has been used on ionospheric plasmas for a long time. The use of this technique for laboratory plasmas was first proposed by Golant ${ }^{133}$ and later implemented on T-10 by Vershkov. ${ }^{134}$

Hugenholtz ${ }^{82,83}$ and others ${ }^{41,133,135,138}$ have proposed using an ultrafast microwave radar pulse to determine the plasma density. They proposed sending into the plasma a square radar pulse of waves composed of a broad band of frequencies. The reflection of the different frequencies at different density layers will change the 
shape of the returning radar pulse. The deformation on the pulse signal is due to the response of the square law detector to higher order derivatives of the phase delay versus frequency, and spectra deformation by dispersive effects of the waveguides and plasma. This presents some problems for pulse reflectometry, because of the broad spectrum of the square pulse. Nevertheless, from the new shape of the reflected pulse, the information on the density can be decoded. Computer simulations of this concept indicates that a pulse rise/fall time on the order of 200 picoseconds will be required to see any measurable change. For time delay measurements, a rise/fall time of about 1 nanosecond is necessary to get a reflected pulse of the same shape as the incident pulse.

\subsubsection{Other techniques}

Measurement of magnetic fluctuations Recent research by Bretz and Vahala, ${ }^{113,114}$ and others ${ }^{69-71,73,74}$ has suggested that the fluctuations in the magnetic field can be determined by the study of the $O \rightarrow X$ mode conversion. The interested reader is referred to the original articles in the references.

Small scale edge fluctuations It has been proposed by García ${ }^{110}$ that the spatial location of small scale fluctuations at the edge of a fusion plasma can be determined by analyzing the enhanced scattering of a reflectometry signal on the reflection layer at the edge of the plasma, where the nonhomogeneous fluctuations of the plasma are strong. For his theory, García considers a slab model and an O-mode of propagation for the wave. 


\subsection{Measuring Density Fluctuations}

Reflectometry works on the principle of the total reflection of an electromagnetic wave by a cutoff layer in a plasma. Such reflection occurs at the point where the inc of refraction becomes equal to zero, i.e., $n^{2}=0$.

The basic principle of the reflectometer is shown in Figure 2.5. From this basic schematic it can be seen that the signal from the source is divided into two signals: the reference signal and the probing signal. The probing signal is launched into the plasma, and as the wave propagates through the plasma, the index of refraction decreases until it becomes zero, at which point the wave is reflected back, collected by a second antenna, and fed into the phase detector to be compared in phase with the reference signal.

Analytically, the process can be seen as follows. First, ignore the effect of the plasma in front of the reflection layer on the incident wave. Under this assumption the signal path length, $L_{\boldsymbol{\theta}}$, is equal to a certain number of wavelengths, call it $a$, then:

$$
\begin{aligned}
L_{0} & =a \lambda \\
& =\frac{\phi_{a}}{2 \pi} \lambda
\end{aligned}
$$

A similar argument is true for the signal in the reference path, that is:

$$
\begin{aligned}
L_{r} & =b \lambda \\
& =\frac{\phi_{r}}{2 \pi} \lambda
\end{aligned}
$$

where $\phi_{\mathrm{r}}$ and $\phi_{r}$ are the probing signal phase and the reference signal phase, respectively. Solving for the phases and using $c=\lambda f$ gives:

$$
\begin{aligned}
& \phi_{.}=L_{\mathbf{t}} \frac{\omega}{c} \\
& \phi_{r}=L_{r} \frac{\omega}{c}
\end{aligned}
$$




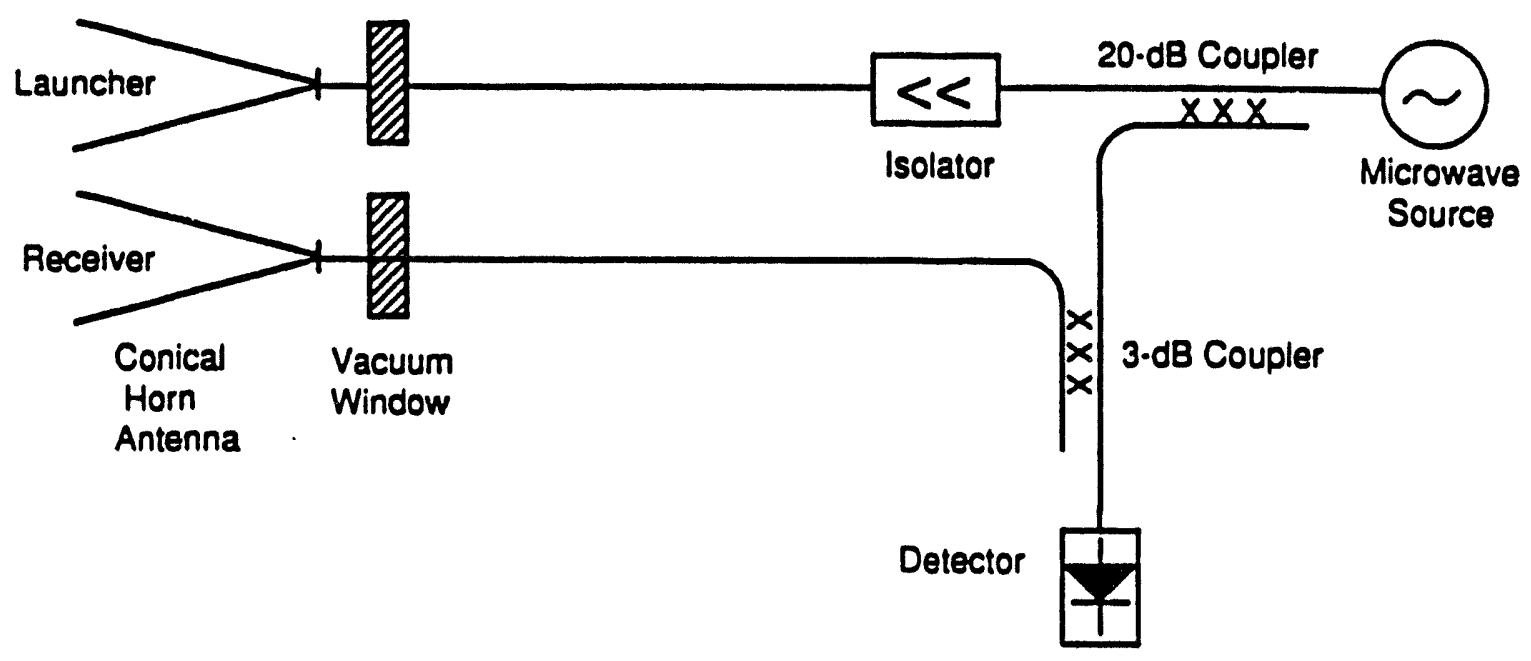

Figure 2.5: Basic schematic of a reflectometer. 
Then the phase difference at the detector becomes:

$$
\phi=\phi_{1}-\phi_{r}=\left(L_{1}-L_{r}\right) \frac{\omega}{c}
$$

where $\phi$ is the phase difference between the probing signal and the reference signal.

For a reflectometer, a change in the phase difference, $\delta \phi$, can be produced by a change in frequency, $\omega$, or by a change and/or motion of the reflection layer, thus producing a change in the signal path length, $L$. The reference path remains electrically constant. Then using variational analysis, the change in the phase difference, $\delta \phi$, is given by:

$$
\delta \phi=\delta\left(L_{\bullet}-L_{r}\right) \frac{\omega}{c}+\left(L_{\bullet}-L_{r}\right) \frac{\delta \omega}{c}
$$

From this equation it can be seen that in the case that the frequency, $\omega$, is held constant $(\delta \omega=0)$, the changes in the phase difference can only come from changes in the probing signal path, $L_{2}$, and this is a direct measurement of the motion of the reflection layer. For the other mode, in which it is assumed that there are no changes in the probing signal path $\left(\delta\left(L_{0}-L_{r}\right)=0\right)$, the changes in the phase difference can only come from the change in frequency, $\delta \omega$.

Thus in the fixed frequency $(\delta \omega=0)$ mode of operation, the changes in phase difference can be used to measure changes (fluctuations) in the density layer. While in the swept frequency mode (assuming $\delta\left(L_{0}-L_{r}\right)=0$ ) the value of $L_{\mathfrak{a}}-L_{r}$ can be calculated, and the position of the density layer and hence the density profile can also be calculated.

\subsubsection{Using the O-mode}

Because of the single dependence on the density, the availability of frequency sources in the range of the 0 -mode, and the simple form of the index of refraction, equation 2.62 , the 0 -mode was the first to gain popularity in the fusion community. Among the first to work with the 0 -mode were Anisimov, ${ }^{17}$ Anoshkin, ${ }^{18}$ and 
Colchin. ${ }^{19,20}$ Some modern fusion experiments using O-mode reflectometers are TFR, ${ }^{21,76,77}$ JET, ${ }^{80,70,130}$ TFTR, ${ }^{100}$ PLT, ${ }^{70}$ ASDEX, ${ }^{124,128}$ DIII-D, ${ }^{92-80,130,142}$ RTP, ${ }^{24}$ JT-60, ${ }^{131}$ TEXT, $^{130}$ and ATF ${ }^{84,85}$

\subsubsection{Using the $X$-mode}

Because of the dependence on both the density and the magnetic field, and the complicated form of the index of refraction, equation $2.63, \mathrm{X}$-mode reflectometry was explored later than $\mathrm{O}$-mode. Some users of X-mode reflectometers are TFTR, ${ }^{106,107}$ Wendelstein VII-AS, ${ }^{20,31}$ ATF, ${ }^{84,85}$ DIII-D, ${ }^{84-87}$ ASDEX, ${ }^{124-127}$ Uragan$3 \mathrm{M},{ }^{123}$ and Petula-B. ${ }^{43}$

\subsection{Measuring the Density Profile}

\subsubsection{Proflle reconstruction using the 0 -mode}

The reconstruction of the density profile using the $\mathrm{O}$-mode is achieved with an Abel inversion technique. $3,8,87,84$ The derivation starts with equation 2.88 :

$$
\phi(\omega)=\frac{2 \omega}{c} \int_{r_{\mathrm{c}}(\omega)}^{r_{0}} n(r, \omega) d r-\frac{\pi}{2}
$$

which represents the phase change of a probing wave of frequency $\omega$ after reflection on the cutoff layer. Proceed by differentiating with respect to the probing frequency, $\omega$, to obtain:

$$
\begin{aligned}
\frac{d \phi(\omega)}{d \omega} & =\frac{d}{d \omega}\left(\frac{2 \omega}{c} \int_{r_{c}(\omega)}^{r_{0}} n(r, \omega) d r-\frac{\pi}{2}\right) \\
& =\frac{2}{c}\left[\int_{r_{c}(\omega)}^{r_{0}} \frac{d}{d \omega}(\omega n(r, \omega)) d r-\omega n\left(r_{c}(\omega), \omega\right) \frac{d r_{c}(\omega)}{d \omega}\right]
\end{aligned}
$$

using the cutoff condition at $r_{c}(\omega)$ for $\omega$, gives $n^{2}\left(r_{c}(\omega), \omega\right)=0$, so that the second term above vanishes as long as $\frac{d r_{c}(\omega)}{d \omega}$ is bounded. Now equation 2.142 transforms 
into:

$$
\frac{d \phi(\omega)}{d \omega}=\frac{2}{c} \int_{r_{0}(\omega)}^{r_{n}} \frac{d}{d \omega}(\omega n(r, \omega)) d r
$$

It has been shown by Budden ${ }^{5}$ that a packet of waves of frequency $\omega$ travels with velocity $V_{z}$ parallel to the density gradient, where $V_{z}$ is given by:

$$
\frac{c}{V_{z}}=\frac{d}{d \omega}(\omega n(r, \omega))
$$

where $V_{a}$ is the component of the group velocity, $V_{g}$, parallel to the density gradient of the plasma. Then the group refractive index is defined as:

$$
\begin{aligned}
n^{\prime}(r, \omega) & =\frac{c}{V_{g}} \\
& =\frac{d}{d \omega}(\omega n(r, \omega))
\end{aligned}
$$

which allows equation 2.143 to be written as:

$$
\frac{d \phi(\omega)}{d \omega}=\frac{2}{c} \int_{r_{c}(\omega)}^{r_{0}} n^{\prime}(r, \omega) d r
$$

and

$$
\tau=\frac{d \phi(\omega)}{d \omega}
$$

where $\tau$ is defined as the delay time. ${ }^{137}$

For the particular case of the $\mathrm{O}$-mode, the refractive index is given by equation 2.62 as:

$$
n_{O}(r, \omega)=\left(1-\frac{\omega_{p e}^{2}(r)}{\omega^{2}}\right)^{\frac{1}{2}}
$$

where the plasma frequency, $\omega_{p e}$; is related to the local electron density by equation 2.34:

$$
\omega_{p e}^{2}(r) \equiv \frac{n_{e}(r) q_{e}^{2}}{m_{e} \epsilon_{0}}
$$

and the probing frequency, $\omega$, is given by a similar equation:

$$
\omega^{2} \equiv \frac{n_{e}\left(r_{c}\right) q_{e}^{2}}{m_{e} \epsilon_{0}}
$$


where $n_{\mathrm{e}}\left(r_{\mathrm{c}}\right)$ is the density at the reflection layer for the probing frequency $\omega$ (or the cutoff density or the critical density).

From equation 2.149 the group refractive index can be derived, that is:

$$
n^{\prime}(r, \omega)=\left(1-\frac{\omega_{p e}^{2}(r)}{\omega^{2}}\right)^{-\frac{1}{2}}
$$

Substituting into equation 2.147 yields:

$$
\frac{d \phi(\omega)}{d \omega}=\frac{2}{c} \int_{r_{e}(\omega)}^{r_{0}}\left(1-\frac{\omega_{p e}^{2}(r)}{\omega^{2}}\right)^{-\frac{1}{2}} d r
$$

or

$$
\frac{d \phi(\omega)}{d \omega}=\frac{2}{c} \int_{r_{e}(\omega)}^{r_{0}}\left(\frac{\omega^{2}}{\omega^{2}-\omega_{p e}^{2}(r)}\right)^{\frac{1}{2}} d r
$$

A change of variable on the integration, from $r \rightarrow \omega_{p e}$, can be performed, since:

$$
r=r\left(\omega_{p e}\right)
$$

and vice versa:

$$
\omega_{p e}=\omega_{p e}(r)
$$

Thus equation 2.154 becomes:

$$
\frac{1}{\omega} \frac{d \phi(\omega)}{d \omega}=\frac{2}{c} \int_{\omega}^{0} \frac{1}{\left(\omega^{2}-\omega_{p e}^{2}\right)^{\frac{1}{2}}} \frac{d r}{d \omega_{p e}} d \omega_{p e}
$$

This transforms it into an Abel-like form:

$$
\begin{aligned}
\frac{1}{2 \omega} \frac{d \phi(\omega)}{d \omega} & =\frac{1}{c} \int_{\omega}^{0} \frac{1}{\left(\omega^{2}-\omega_{p e}^{2}\right)^{\frac{1}{2}}} \frac{d r}{2 \omega_{p e} d \omega_{p e}} 2 \omega_{p e} d \omega_{p e} \\
\frac{d \phi(\omega)}{d\left(\omega^{2}\right)} & =\frac{1}{c} \int_{\omega}^{0} \frac{1}{\left(\omega^{2}-\omega_{p e}^{2}\right)^{\frac{1}{2}}} \frac{d r}{d\left(\omega_{p e}^{2}\right)} d\left(\omega_{p e}^{2}\right)
\end{aligned}
$$

Substituting $\omega_{p e}^{2} \rightarrow t, \omega^{2} \rightarrow x$, gives:

$$
\frac{d \phi(x)}{d x}=\frac{-1}{c} \int_{0}^{x} \frac{1}{(x-t)^{\frac{1}{2}}} \frac{d r(t)}{d t} d t
$$


By making the substitutions:

$$
f(x)=\frac{d \phi(x)}{d x}
$$

and

$$
\varphi(t)=\frac{-1}{c} \frac{d r(t)}{d t}
$$

one obtains the Abel equation given in Arfken: ${ }^{47}$

$$
f(x)=\int_{0}^{x} \frac{\varphi(t)}{(x-t)^{\frac{1}{2}}} d t
$$

with solution:

$$
\varphi(x)=\frac{1}{\pi} \frac{d}{d x} \int_{0}^{x} \frac{f(t)}{(x-t)^{\frac{1}{2}}} d t
$$

Substituting equations 2.161 and 2.162 into equation 2.164 gives:

$$
\frac{-1}{c} \frac{d r(x)}{d x}=\frac{1}{\pi} \frac{d}{d x} \int_{0}^{x} \frac{1}{(x-t)^{\frac{1}{2}}}\left(\frac{d \phi(t)}{d t}\right) d t
$$

Integrating with respect to $x$, gives:

$$
r(x)-r_{0}=\frac{-c}{\pi} \int_{0}^{x} \frac{1}{(x-t)^{\frac{t}{2}}}\left(\frac{d \phi(t)}{d t}\right) d t
$$

Substituting $\omega_{p e}^{2}=t$ and $\omega^{2}=x$, then yields:

$$
r(\omega)=r_{0}-\frac{c}{\pi} \int_{0}^{\omega} \frac{1}{\left(\omega^{2}-\omega_{p e}^{2}\right)^{\frac{1}{2}}}\left(\frac{d \phi\left(\omega_{p e}\right)}{d \omega_{p e}}\right) d \omega_{p e}
$$

which is the final form of the reconstruction formula.

Note that in order to reconstruct the radius it is necessary to know the derivative of the phase delay with respect to the frequency, $\left(\frac{d \phi\left(\omega_{\text {end }}\right)}{d \omega_{\text {pe }}}\right)$, rather than the phase delay itself. This is accomplished easily using the identity:

$$
\begin{aligned}
\frac{d \phi(\omega)}{d \omega} & =\frac{d \phi}{d t} \frac{d t}{d \omega} \\
& =\frac{d \phi}{d t}\left(\frac{d \omega}{d t}\right)^{-1}
\end{aligned}
$$


or by other numerical means. Another point to notice is that the integral starts at $0 \mathrm{~Hz}$. A sweep from $0 \mathrm{~Hz}$ to some $50 \mathrm{GHz}$ is an impossible task for any known hardware at this time. A way to avoid this problem is to assume a density profile that corresponds to the frequencies from $0 \mathrm{~Hz}$ to some more reasonable $\omega_{\text {low }}$ such that the corresponding region of the integral can be calculated numerically and the frequency band is narrow enough for the hardware limitations.

\subsubsection{Profile reconstruction using the $\mathrm{X}$-mode}

Full phase technique The refractive index for the X-mode depends on both the density profile and the magnetic field, as shown in equation 2.63:

$$
\begin{aligned}
n_{X}^{2} & =1-\frac{\omega_{p e}^{2}}{\omega^{2}} \frac{\omega^{2}-\omega_{p e}^{2}}{\omega^{2}-\omega_{p e}^{2}-\omega_{e c}^{2}} \\
\omega_{p e}^{2}(r) & =\frac{n_{e}(r) q_{e}^{2}}{m_{e} \epsilon_{0}} \\
\omega_{e c}(r) & =\frac{q_{e} B(r)}{m_{e}}
\end{aligned}
$$

where $\omega_{\text {pe }}(r)$ carries the dependence on the density and $\omega_{\text {ec }}(r)$ carries the dependence on the magnetic field. Therefore an analytical inversion such as the one for the O-mode is not known, and numerical methods are necessary to extract the solution.

A particularly nice method has been developed by Doyle ${ }^{94,97}$ and will be explained here. Doyle starts by assuming a knowledge of the density profile up to the $j^{\text {th }}$ frequency of the probing array. Then the phase delay for the probing wave of frequency $\omega_{j}$ is given by equation 2.88 :

$$
\phi\left(\omega_{j}\right)=\frac{2 \omega_{j}}{c} \int_{r_{e}\left(\omega_{j}\right)}^{r_{0}} n\left(r, \omega_{j}\right) d r
$$

where the order of the limits has been changed to ensure a positive phase and a term of $\frac{\pi}{2}$ has been dropped. 
For the next frequency, $\omega_{j+1}$, Doyle represents the measured phase by the equation:

$$
\begin{aligned}
\phi\left(\omega_{j+1}\right)= & \frac{2 \omega_{j+1}}{c}\left\{\int_{r_{c}\left(\omega_{j}\right)}^{r_{0}} n\left(r, \omega_{j+1}\right) d r\right. \\
& \left.+\frac{1}{2}\left[n\left(r_{c}\left(\omega_{j}\right), \omega_{j+1}\right)+n\left(r_{c}\left(\omega_{j+1}\right), \omega_{j+1}\right)\right] \Delta r\left(\omega_{j+1}\right)\right\}
\end{aligned}
$$

where $\Delta r\left(\omega_{j+1}\right)$ is the increment in radial distance between the reflection point of $\omega_{j}$ and the reflection point of $\omega_{j+1}$. This equation is like a Simpson's rule representation of the integral of equation 2.173 for the frequency $\omega_{j+1}$. Now, by definition of the cutoff condition:

$$
n\left(r_{c}\left(\omega_{j+1}\right), \omega_{j+1}\right)=0
$$

and from equation 2.174 the increment on $r$ of the reflection point can be solved as:

$$
\Delta r\left(\omega_{j+1}\right)=2 \frac{\phi\left(\omega_{j+1}\right) \frac{c}{2 \omega_{j+1}}-\int_{r_{c}\left(\omega_{j}\right)}^{r_{0}} n\left(r, \omega_{j+1}\right) d r}{n\left(r_{c}\left(\omega_{j}\right), \omega_{j+1}\right)}
$$

From this the new point of reflection for frequency $\omega_{j+1}$ can be calculated, that is:

$$
r_{c}\left(\omega_{j+1}\right)=r_{c}\left(\omega_{j}\right)+\Delta r\left(\omega_{j+1}\right)
$$

and the density of the cutoff at this point can be calculated from the cutoff condition on the refractive index, i.e., a density can be found such that:

$$
n\left(r_{c}\left(\omega_{j+1}\right), n_{e j+1}, \omega_{j+1}\right)=0
$$

to obtain the density at the cutoff point, $r_{c}\left(\omega_{j+1}\right)$. Now this new cutoff point, $r_{c}\left(\omega_{j+1}\right)$, and density, $n_{e}\left(\omega_{j+1}\right)$, can be incorporated into the density profile and the process repeated to the next frequency in an inductive way.

Phase difference technique In the dual frequency phase difference technique the phase difference data can be seen as the parts of a puzzle, which when put together in the right order will give the desired picture. To elaborate on this, 
start by assuming knowledge of the position $R_{1}$ at which the first frequency of the frequency array, $\omega_{1}$, is reflected. This $R_{1}$ can be the position of the outer most flux surface or the scrapeoff layer of the limiter. From this assumption the value of the density at $R_{1}$ can be calculated, this is $n_{e 1}=n_{e}\left(R_{1}\right)$, by using the cutoff condition, i.e., $n\left(R_{1}, n_{e 1}, \omega_{1}\right)=0$. Now that there is an $R_{1}, n_{e 1}$, and $\omega_{1}$, one can assume a linear model ${ }^{94,97}$ for the density from the edge of the plasma (where the density $\left.n_{e} \approx 0\right)$ to the first point of reflection $\left(R_{1}, n_{e 1}\right)$, such that Doyle's method can be used and the full phase data can be reconstructed by using the equation:

$$
\phi_{j+1}=\phi_{j}+\Delta \phi_{j}
$$

where $\Delta \phi_{j}$ is the phase difference data. 


\subsection{Complications and Error Sources}

The validity of the equations for the dielectric tensor, equation 2.36 , dispersion relation, equation 2.49 , etc; is governed by the validity of the WKB approximation and the geometrical optics approximation. Many conditions in the plasma such as electron cyclotron resonance (ECR) absorption, tunneling, magnetic shear, Doppler effect, movement of the reflection layer with time, etc; can produce breakdown of the initial assumptions and complicate the data beyond recognition. These conditions and their effect on the phase of the reflecting wave will be discussed next. $21,43,69,79,84,85,94-97,136,141$

\subsubsection{Electron Cyclotron Resonance absorption}

Electron Cyclotron Resonance (ECR) absorption of an electromagnetic wave in the plasma occurs when the frequency of the probing wave matches the natural frequency (or the harmonics) of gyration of the electrons at a particular magnetic field strength in the plasma. For the case of toroidal fusion plasmas, the probing wave is launched from the low field side of the plasma in the direction of increasing field and density. For this case there are four possible resonances of concern, two for the O-mode and two for the X-mode. As an example, characteristic cutoff and resonance frequencies for a JT-1 plasma along the minor radius in the equatorial plane and assuming a parabolic density profile with $n_{e}(0)=1.0 \times 10^{10} \mathrm{part} / \mathrm{m}^{3}$ and $B_{T}(0)=1$ Tesla are shown ${ }^{68}$ in Figure 2.6. The curves are as follows:

A. Upper (fast wave, $\omega_{X_{+}}$) and Lower (slow wave, $\omega_{X_{-}}$) X-mode cutoff frequencies, given by equation 2.67 :

$$
\omega_{X+}=\frac{\omega_{e c}}{2}+\left(\omega_{p e}^{2}+\left(\frac{\omega_{e c}}{2}\right)^{2}\right)^{\frac{1}{2}}
$$




$$
\omega_{X-}=-\frac{\omega_{e c}}{2}+\left(\omega_{p e}^{2}+\left(\frac{\omega_{e c}}{2}\right)^{2}\right)^{\frac{1}{2}}
$$

B. Plasma frequency, given by equation 2.34 :

$$
\omega_{p e}^{2}=\frac{n_{e} q_{e}^{2}}{m_{e} \epsilon_{0}}
$$

C. First and second harmonics of the electron cyclotron frequency, given by equation 2.26:

$$
\omega_{e c}=\frac{q_{e} B_{0}}{m_{e}}
$$

D. The Upper Hybrid frequency, given by equation 2.65 :

$$
\omega_{U H}^{2}=\omega_{p e}^{2}+\omega_{e c}^{2}
$$

For the $\mathrm{O}$-mode there is the electron cyclotron resonance and its harmonics, given by equation 2.26. While for the X-mode there is the Upper and Lower Hybrid resonance frequencies, given by equations 2.65 and 2.66. For the particular case of the X-mode:

$$
\omega_{L H}<\omega_{U H}<\omega_{X}
$$

where $\omega_{X}$ is the cutoff frequency of the $X$-mode and the right inequality $\omega_{U H}<\omega_{X}$ can be shown as follows:

$$
\begin{aligned}
\left(\omega_{p e}^{2}+\omega_{e c}^{2}\right)^{\frac{1}{2}} & <\frac{\omega_{e c}}{2}+\left(\omega_{p e}^{2}+\left(\frac{\omega_{e c}}{2}\right)^{2}\right)^{\frac{1}{2}} \\
\omega_{e c}^{2} & <\frac{\omega_{e c}^{2}}{2}+\omega_{e c}\left(\omega_{p e}^{2}+\left(\frac{\omega_{e c}}{2}\right)^{2}\right)^{\frac{1}{2}} \\
\frac{\omega_{e c}}{2} & <\left(\omega_{p e}^{2}+\left(\frac{\omega_{e c}}{2}\right)^{2}\right)^{\frac{1}{2}} \\
0 & <\omega_{p e}^{2}
\end{aligned}
$$

This shows that for the $\mathrm{X}$-mode the probing wave will always reach the cutoff layer: before it can find the absorption layer, except at the very edge of the plasma, where 
A. Lower (slow wave) and upper (fast wave) cutoff frequency, given by equation 2.67 .

B. Plasma frequency, given by equation 2.34 .

C. First and second harmonics of the electron cyclotron frequency, given by equation 2.26.

D. The Upper Hybrid frequency, given by equation 2.65 .

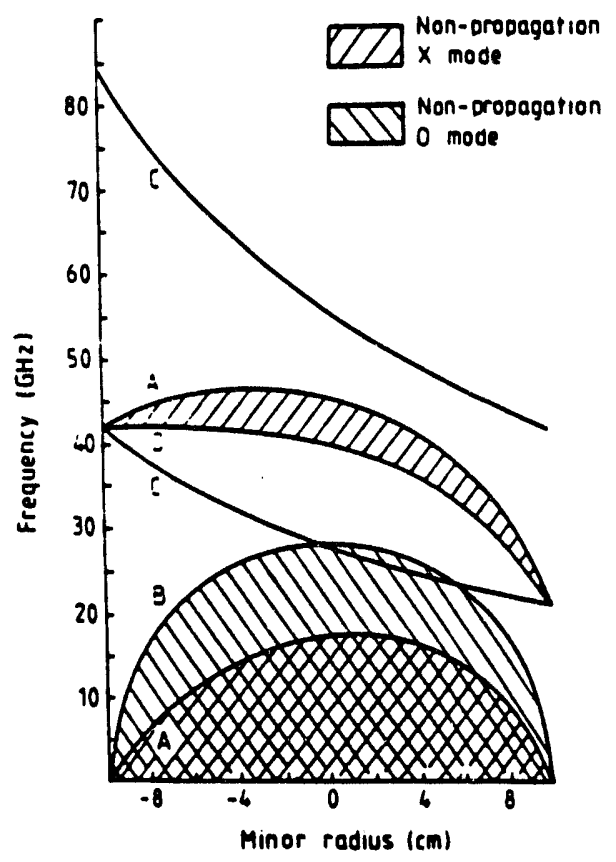

Figure 2.6: Typical cutoff and resonance frequencies on JT-1. 
the resonance and the cutoff are so close together that some of the power may be absorbed because of tunneling ${ }^{5,44}$ and other coupling effects. Another problem at the edge of the plasma is the validity of the geometrical optics approximation condition, equation 2.84:

$$
\frac{\omega}{c} \frac{n^{2}}{\left|\frac{d n}{d r}\right|}=\frac{2 \pi L_{n}}{\lambda} \gg 1
$$

where $L_{n} \equiv n^{2}\left|\frac{d n}{d r}\right|^{-1}$ is the gradient scale length of the refraction index, $n$. This condition states that in order for the geometric optics approximation to be valid, the gradient scale length of the index of refraction must be larger than the wavelength of the probing wave.

From the above formulae it can be seen that the lower cutoff (slow wave) and upper hybrid will not interfere with the probing wave, but that the second harmonic of the electron cyclotron resonance may impair the ability to probe the far minor radius of the plasma because of absorption of the probing wave by the the second harmonic of the electron cyclotron resonance at the plasma edge. As an example, using Figure 2.6, for a probing frequency of $45 \mathrm{GHz}$ it can be seen that the wave will be absorbed at the edge of the plasma by the second harmonic of the electron cyclotron resonance and little power will be able to reach the upper cutoff (fast wave) of the $\mathrm{X}$-mode at the inner part of the plasma. Absorption at higher harmonics may be reached, but its coupling to the plasma is usually considered in general weak. ${ }^{86,51}$

\subsubsection{Tunneling}

Early in this chapter, page 34, the solution of the wave equation at the reflecting layer was discussed. In order to obtain a solution it was assumed that the density was increasing linearly at the point of reflection. This condition is embodied in equation 2.85 and it is valid throughout most of the plasma except at the point 
where the density peaks or flattens out. In this region of flat density the density gradient goes to 0 , while the width of the reflection layer and the curvature of the profile becomes large, and the condition for quasi-linearity, equation 2.85 , is not valid.

Under the condition of tunneling, a fraction of ine vave power will be reflected and another fraction will pass through the point of reflection. The fraction of wave that tunnels may be reflected by the vacuum wall or at some other part of the plasma, producing mixing of the real signal and a phantom signal in the detector, thus complicating the extraction of the density profile.

K. G. Budden ${ }^{5}$ (1955) also analyzed the interesting occurrence of back-toback linear and singular turning points. ${ }^{53,44}$ This physical situation occurs in our case when the $\mathrm{X}$-wave propagates to the reflection point and tunnels through the evanescent region to reach the Upper Hybrid resonance layer, as it may occur at the very edge of a fusion plasma. Budden considered the wave equation in the form:

$$
\frac{d^{2} E}{d x^{2}}+\left(\frac{\gamma}{x}+\frac{\gamma^{2}}{\eta^{2}}\right) E=0
$$

and used the substitutions:

$$
\xi=\frac{ \pm 2 i \gamma x}{\eta}
$$

to reduce the equation to the standard form given by E. T. Whittaker and G. N. Watson $^{56}$ (1927) for the confluent hypergeometric function. This gives left and right running waves. The right moving wave passes the zero first and then comes to the singularity and suffers reflection, transmission, and absorption. The coefficients of reflection $(R)$ and of transmission $(T)$ are:

$$
\begin{aligned}
& |R|=1-\exp (-\pi \eta) \\
& |T|=\exp \left(-\frac{1}{2} \pi \eta\right)
\end{aligned}
$$

where $\eta$ is given by: ${ }^{53}$

$$
\eta=\left|k_{\infty} \Delta x\right|
$$


and it represents the number of free space wavelengths (i.e., the wavelength as $|x| \rightarrow \infty)$ between the point of $n=0$ and the singularity. ${ }^{53}$ When the number of wavelengths is small, appreciable tunneling occurs through the evanescent region. When the spacing is large, no tunneling occurs, and the reflection or absorption of the wave is determined entirely by the first turning point encountered by the wave. For the conditions expected in TFTR the reflection and transmission coefficients are plotted in Figure 2.7 for a typical $n_{e} \propto\left(1-\left(\frac{r}{a}\right)^{\alpha}\right)^{\beta}$ density profile plasma and a toroidal field current of $I_{t f}=67 \mathrm{KAmps}$ which gives a magnetic field of $B_{0}=4.5$ Teslas.

For conditions of very low density (as in the edge of the plasma) and where $\omega_{e c} \gg \omega_{p e}$, an expression for the radial spacing in between the Upper Hybrid and the reflection layers is possible. First express $\omega_{U H}$ and $\omega_{X}$ using the binomial expansion theorem and keeping the first order terms, that is, for $\omega_{U H}$ :

$$
\begin{aligned}
\omega_{U H} & =\left(\omega_{e c}^{2}+\omega_{p e}^{2}\right)^{\frac{1}{2}} \\
& =\omega_{e c}+\frac{1}{2} \frac{\omega_{p e}^{2}}{\omega_{e c}}
\end{aligned}
$$

and similarly for $\omega_{X}$ :

$$
\begin{aligned}
\omega_{X} & =\frac{\omega_{e c}}{2}+\left(\omega_{p e}^{2}+\left(\frac{\omega_{e c}}{2}\right)^{2}\right)^{\frac{1}{2}} \\
& =\omega_{e c}+\frac{\omega_{p e}^{2}}{\omega_{e c}}
\end{aligned}
$$

where only the first order terms have been kept.

Next, for any given probing frequency, $\omega$, there is the condition:

$$
\omega_{U H}(R)=\omega_{X}(R+\Delta R)
$$

which represents the matching condition of the probing wave at both the reflecting layer and the resonance layer. Now, by substituting equations 2.195 and 2.193 into 

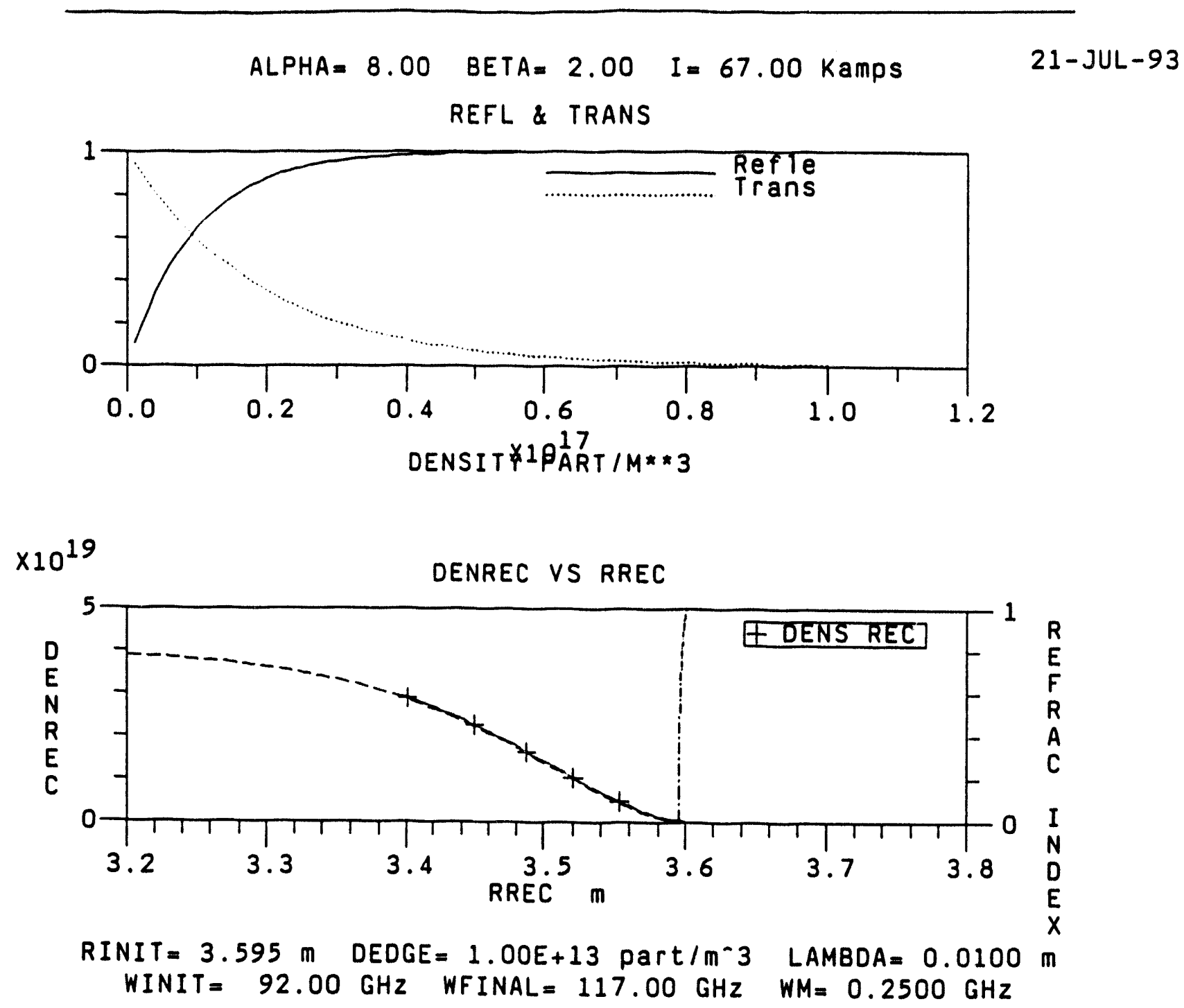

Figure 2.7: Reflection and transmission coefficients for a typical TFTR plasma (upper plot). The assumed density profile is included (lower plot) and it is of the from $n_{e} \propto\left(1-\left(\frac{r}{a}\right)^{\alpha}\right)^{\beta}$ where all the parameters have been defined before. 
equation 2.196 above and using the definitions for the $\omega_{\text {pe }}$ and $\omega_{\text {ec }}$ (equations 2.34 and 2.26), one finds:

$$
\frac{q_{e} B_{0} R_{0}}{m_{e} R}+\frac{1}{2} \frac{q_{e} n_{e}(R) R}{\epsilon_{0} B_{0} R_{0}}=\frac{q_{e} B_{0} R_{0}}{m_{e}(R+\Delta R)}+\frac{q_{e} n_{e}(R+\Delta R)(R+\Delta R)}{\epsilon_{0} B_{0} R_{0}}
$$

Using the binomial expansion on the term $(R+\Delta R)^{-1}$ yields:

$$
\frac{q_{e} B_{0} R_{0}}{m_{e} R}+\frac{1}{2} \frac{q_{e} n_{e}(R) R}{\epsilon_{0} B_{0} R_{0}}=\frac{q_{e} B_{0} R_{0}}{m_{e} R}-\frac{q_{e} B_{0} R_{0} \Delta R}{m_{e} R^{2}}+\frac{q_{e} n_{e}(R+\Delta R)(R+\Delta R)}{\epsilon_{0} B_{0} R_{0}}
$$

which can be simplified to:

$$
\frac{B_{0} R_{0} \Delta R}{m_{e} R^{2}}=\frac{\left(n_{e}(R+\Delta R)-\frac{1}{2} n_{e}(R)\right) R}{\epsilon_{0} B_{0} R_{0}}+\frac{n_{e}(R+\Delta R) \Delta R}{\epsilon_{0} B_{0} R_{0}}
$$

for a final result of:

$$
\Delta R=\frac{\frac{\left(n_{e}(R+\Delta R)-\frac{1}{2} n_{e}(R)\right) R}{\epsilon_{0} B_{0} R_{0}}}{\frac{B_{0} R_{0}}{m_{e} R^{2}}-\frac{n_{e}(R+\Delta R)}{\epsilon_{0} B_{0} R_{0}}}
$$

which holds at the edge of the plasma, where the density is small enough such that $\omega_{e c} \gg \omega_{p e}$. For a TFTR plasma, with an edge density of $n_{e}(a) \approx 0.1 \times 10^{10} \frac{\text { part }}{\mathrm{m}^{\mathrm{j}}}$ the separation is $\Delta R \approx 1.5 \mathrm{~cm}$. The importance of this result is that it provides an analytical formula for the radial spacing between the upper cutoff (fast wave) of the X-mode and the Upper Hybrid resonance frequency layer on the plasma edge. This explains why the amplitude of the receiving signal tends to be rather small at the beginning of the edge of the plasma and increases for frequencies cutoff farther in the plasma.

It is possible to simplified equation 2.200 a little more to the expense of credibility of the result. This is, by taking the limit as the density goes to 0 a simpler equation can be obtained. In the limit as the density goes to $0, n_{e} \rightarrow 0$, the following substitutions can be done on equation 2.200 :

$$
\lim _{n_{e} \rightarrow 0}\left(n_{e}(R+\Delta R)-\frac{1}{2} n_{e}(R)\right)=\frac{1}{2} n_{e}(R)
$$




$$
\lim _{n_{e} \rightarrow 0}\left(\frac{B_{0} R_{0}}{m_{e} R^{2}}-\frac{n_{e}(R+\Delta R)}{\epsilon_{0} B_{0} R_{0}}\right)=\frac{B_{0} R_{0}}{m_{e} R^{2}}
$$

where the approximation $n_{\mathrm{e}}(R+\Delta R) \approx n_{\mathrm{e}}(R)$ has been used. Then the radial spacing, $\Delta R$, becomes:

or as:

$$
\Delta R \approx \frac{\frac{\frac{1}{2} n_{e}(R) R}{\epsilon_{0} B_{0} R_{0}}}{\frac{B_{0} R_{0}}{m_{e} R^{2}}}
$$

$$
\Delta R \approx \frac{n_{e}(R) m_{e} R^{3}}{2 \epsilon_{0} B_{0}^{2} R_{0}^{2}}
$$

which can be simplified to:

$$
\frac{\Delta R}{R} \approx \frac{1}{2} \frac{\omega_{p e}^{2}}{\omega_{e c}^{2}} R
$$

\subsubsection{Magnetic shear}

For the geometrical optics approximation in section 2.2.5, it was assumed that there is no coupling in between the X-and O-mode of propagation, i.e., that both modes propagate through the plasma independently of each other. This is not always true for fusion plasmas. ${ }^{80,73-75,84,113,122,141}$ The change of the magnetic field angle with respect to the toroidal axis, and therefore the magnetic shear, can produce a coupling of one mode to another. ${ }^{73}$ As an example, assume that an $\mathrm{O}$-mode wave is launched (and therefore with $\vec{E} \| \vec{B}$ ) into the plasma. As the wave propagates, the magnetic field will rotate, producing a component of the $\vec{E}$ field that is parallel to $\vec{B}$ (for an O-mode wave) and an $\vec{E}$ component that is perpendicular to $\vec{B}$ (for an $\mathrm{X}$-mode wave). Therefore, some of the power has been converted from one mode to the other. The resulting mixing of modes makes the interpretation of the data much more difficult, since the two modes have different refractive indexes and therefore different propagation velocities. ${ }^{141}$ In addition, because of the different refraction indexes of the $\mathrm{X}$-mode and $\mathrm{O}$-mode waves, their reflection points and corresponding phase will be different. 
The fact that the electron cyclotron emission is generated in two modes of propagation was first noted by Engelmann and Curatolo. ${ }^{72}$ They suggested that this phenomena could be used to measure the orientation of the magnetic field at the source of the radiation. Clearly, if the electron cyclotron radiation emerged from the plasma with the same orientation that the electric field at the point of the source, then an experimental determination of its orientation will fix the orientation of the magnetic field at the position of the source. Unfortunately, it has been shown that as the radiation propagates from its source through the plasma and across the sheared magnetic field, there is a tendency of the electric vector field to rotate with the magnetic field. ${ }^{73-78}$ Thus, in general, the electric field will not emerge with the same orientation that it had at the position of the source, but at some angle given by the direction of the magnetic field in some low plasma density region near the plasma edge.

In his paper, Boyd ${ }^{74}$ gives a set of equations to determine the boundary where the anisotropy overwhelms the shear effects of the magnetic field. The formula is:

$$
S=\frac{2 c}{\omega} \frac{d \theta}{d r} \approx 2 \frac{\lambda_{0}}{a} \frac{\psi}{\pi}
$$

where $S$ is the shear, $\lambda_{0}$ is the free space wavelength, $a$ is the radius of the plasma and $\psi$ is the maximum value of the angle between the magnetic field and the toroidal axis, $\theta$. Boyd calculates that for a circular cross section tokamak, some typical values are: $\psi \approx \frac{\pi}{30}, \lambda \approx 2 \times 10^{-3} \mathrm{~m}, a \approx 0.5 \mathrm{~m}$, and therefore the value for the shear is $S \approx 3 \times 10^{-4}$. This value for the shear will give a very low critical density, and therefore the direction of polarization of the emission will be rotated due to the magnetic shear. 


\subsubsection{Motion/fluctuation of the cutoff layer}

In section 2.4 the change in phase difference, equation 2.139 , was derived for a probing wave in a simplified propagation model. When the frequency sweep method is used, it is assumed that the density layers do not move; that is:

$$
\delta\left(L_{0}-L_{r}\right)=0
$$

Under such conditions the density profile is easily obtainable. The effect of the motion of the reflection layer is best illustrated by the use of an example. ${ }^{82}$ Assume the following parameters:

- probing frequency, $f=30 \mathrm{GHz}$

- frequency sweep, $\delta f=0.1 \mathrm{GHz}$

- path difference, $L_{4}-L_{\mathrm{r}}=6 \mathrm{~m}$

- change in path difference, $\delta\left(L_{\mathbf{0}}-L_{r}\right)=0.5 \mathrm{~mm}$.

With this set of parameters, equation 2.139 gives a phase difference of $\delta \phi=0.1 \pi+4 \pi$. The term $0.1 \pi$ corresponds to the error associated with the motion of the reflecting layer. The magnitude of this error can lead to serious problems, especially when the frequency is swept slowly over a broad band of frequencies. ${ }^{68,21}$ The way to reduce this error is to increase the sweeping rate and reduce the frequency band.

Doyle ${ }^{07}$ and others ${ }^{124,120}$ report that in measuring the phase delay, $\phi_{p}(f)$, the biggest experimental problem is the one of intrinsic plasma density fluctuations. These density fluctuations add a random Doppler shift to the probing frequency of the measured fringes that can severely distort and/or entirely mask the desired profile information. The effect of this Doppler shift can be minimized by minimizing the sweep time of the probing beam. ${ }^{97}$ Doyle's argument is based on the idea that using a random walk argument, the rms deviation from what the count would be in 
the absent of fluctuations is proportional to $\sqrt{t}$, where $t$ is the sweep time. Therefore, the influence of both forms of phase distortion (fluctuations and Doppler shift) can be minimized by reducing the sweep time, while the desired profile induced phase shift, $\phi$, is independent of the sweep time, $t$.

\subsubsection{Fluctuation correlation length}

The radial spacing between the reflection points of the Upper and Lower side bands depends on the density gradient in between the two points and the density fluctuations around them. If the reflection points are at places in the plasma where the density fluctuations are uncorrelated, then the radial spacing in between them might fluctuate significantly because of the density fluctuations. This depends on the gradient and size of the fluctuations, and the frequency and time resolution of the reflectometer. On the other hand, if the reflection points are at places in the plasma where the density fluctuations are correlated, then the radial spacing in between them may fluctuate only mildly. Thus, to reduce the fluctuation errors in the measurements the local correlation length of the density fluctuations must be bigger then the local radial spacing in between the reflections points of the side bands.

For TFTR, typical correlation lengths are between $2.4 \mathrm{~cm} \cdot{ }^{108-111}$ For a typical TFTR density profile, (i.e., $n_{\varepsilon} \propto\left(1-\left(\frac{r}{a}\right)^{\alpha}\right)^{\beta}$, with $\alpha=8, \beta=2$, and a toroidal field current of $I_{t f}=67 \mathrm{KAmps}$ which gives a magnetic field of $B_{0} \approx 4.5$ Teslas), shown in Figure 2.8 , the model program ${ }^{112}$ shows that the expected side band radial spacing is between 0.25 to $0.5 \mathrm{~cm}$, corresponding to the edge and the peak of the density profile. These are well within acceptable boundaries for the correlation length. ${ }^{108-111}$ 

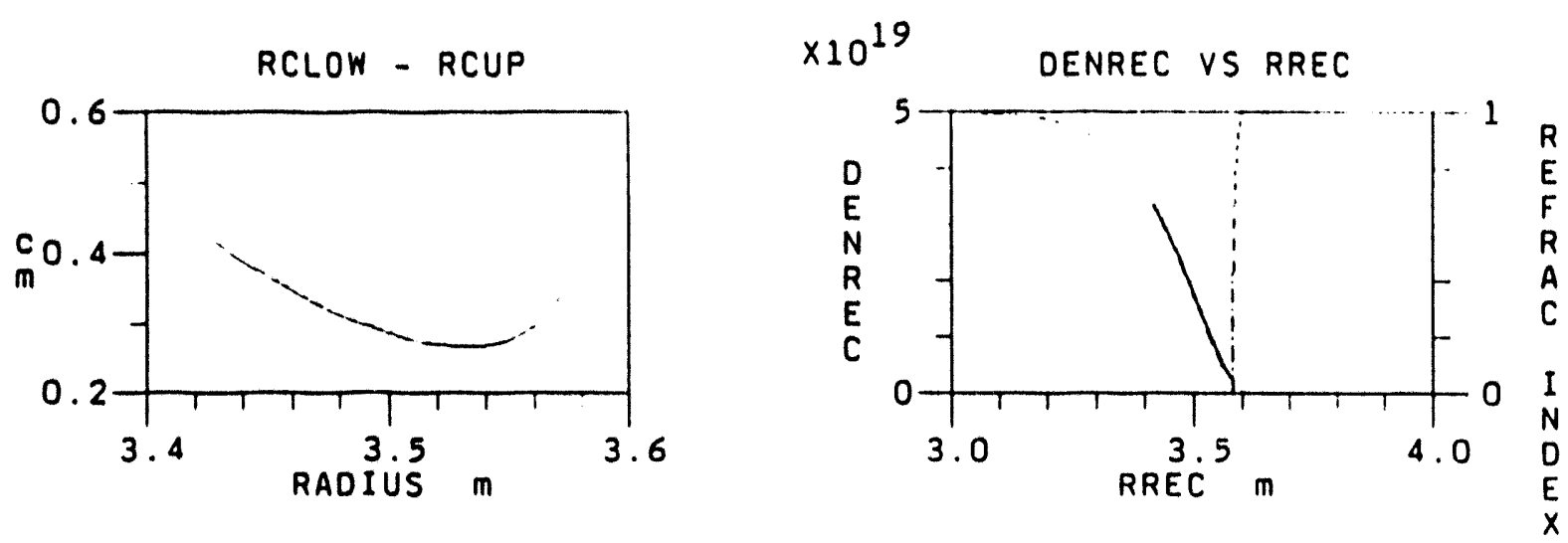

Figure 2.8: Density profile for a typical TFTR plasma and corresponding radial spacing for the probing side bands.

\subsubsection{Relativistic effects}

Even though the temperature of today's fusion plasmas $(10 \mathrm{KeV})$ is small compared to the rest mass energy of the electron $(511 \mathrm{KeV})$, it has been found ${ }^{57-82,80}$ that significant relativistic shifts of the position of the cutoff and modifications of the index of refraction can occur at such low temperatures. This is believed to be caused partially by the high sensitivity of the index of refraction in the vicinity of the cutoff to the plasma response ${ }^{57-81}$ due to simple relativistic mass shift. ${ }^{63}$ It can be shown, too, that the relativistic modifications of the Hermitian part of the dielectric tensor are larger ( $\times 2$ or 3 ) than would be expected from a comparison of the electron temperature with the electron rest mass energy. For the X-mode this can produce a considerable shift of the cutoff $\left(5-10 \mathrm{~cm}\right.$ at $\left.\frac{r}{a} \approx \frac{1}{2}\right)$ and an underestimation of the density profile if the cold plasma model in used..$^{82,63,99}$ For the X-mode the Upper (right) cutoff effect is considerable larger than for the Lower (left) cutoff. ${ }^{03}$ 
The Cold and Hot dielectric models are well known and can be found in references such as: Budden, ${ }^{5}$ Ginzburg, ${ }^{8}$ Swanson, ${ }^{48}$ Bornatici, ${ }^{51}$ Stix,${ }^{53-65}$ and others. ${ }^{52,132}$ The fully relativistic dielectric tensor has been derived by Trubnikov ${ }^{64}$ and by Bornatici ${ }^{51}$ and the weakly relativistic dielectric tensor by Shkarofsky ${ }^{65}$ from Trubnikov's result. The relativistic effect on the index of refraction and the density of the cutoff can be seen in Figure 2.9 for the weakly relativistic model as compared to the cold and hot dielectric models. ${ }^{58-61}$ From plots "a", "b", "c", and "d" it can be seen that the refraction index of the wave for the cold and hot models does not change very much $\left(T_{e} \approx 10 \mathrm{KeV}\right)$. On the other hand, for the weakly relativistic case, there is a significant difference as compared to the cold and hot models, especially for the $\mathrm{X}$-mode of propagation as compared to the O-mode of propagation. That is, from plots " $a$ " and " $b$ " it can be seen that the change in the density of the $\mathrm{O}$-mode cutoff is much less than that for the X-mode. The frequency for plot " $\mathrm{b}$ " is twice that for plot " $\mathrm{a}$ ", and the change in the density of the cutoff is small, while the frequency change in between plots " $c$ " and " $\mathrm{d}$ " is only $10 \mathrm{GHz}$, and the change of the density is much larger as compared to the O-mode. Plot "f" shows the effect of the temperature on the cutoff density for the $\mathrm{X}$-mode of propagation. A significant change in the cutoff density can be observed as the temperature increases. Even at the common plasma temperature value of $T_{e} \approx 10 \mathrm{KeV}$, a non-negligible increase of the cutoff density is observed.

The cutoff is deeper into the plasma for the weakly relativistic model, thus causing an underestimation of the reconstructed density profiles, as can be seen from Figure 2.10..$^{58-61}$ In these plots the curves for the assumed (actual) density profiles are compared with the reconstructed density profiles using the weakly relativistic refractive index for different plasma temperatures for both the $\mathrm{O}$-mode and the X-mode. A significant underestimation on the density profile can be observed, especially for the X-mode, when plots " $\mathrm{a}$ " and "c" are compared with the actual" 


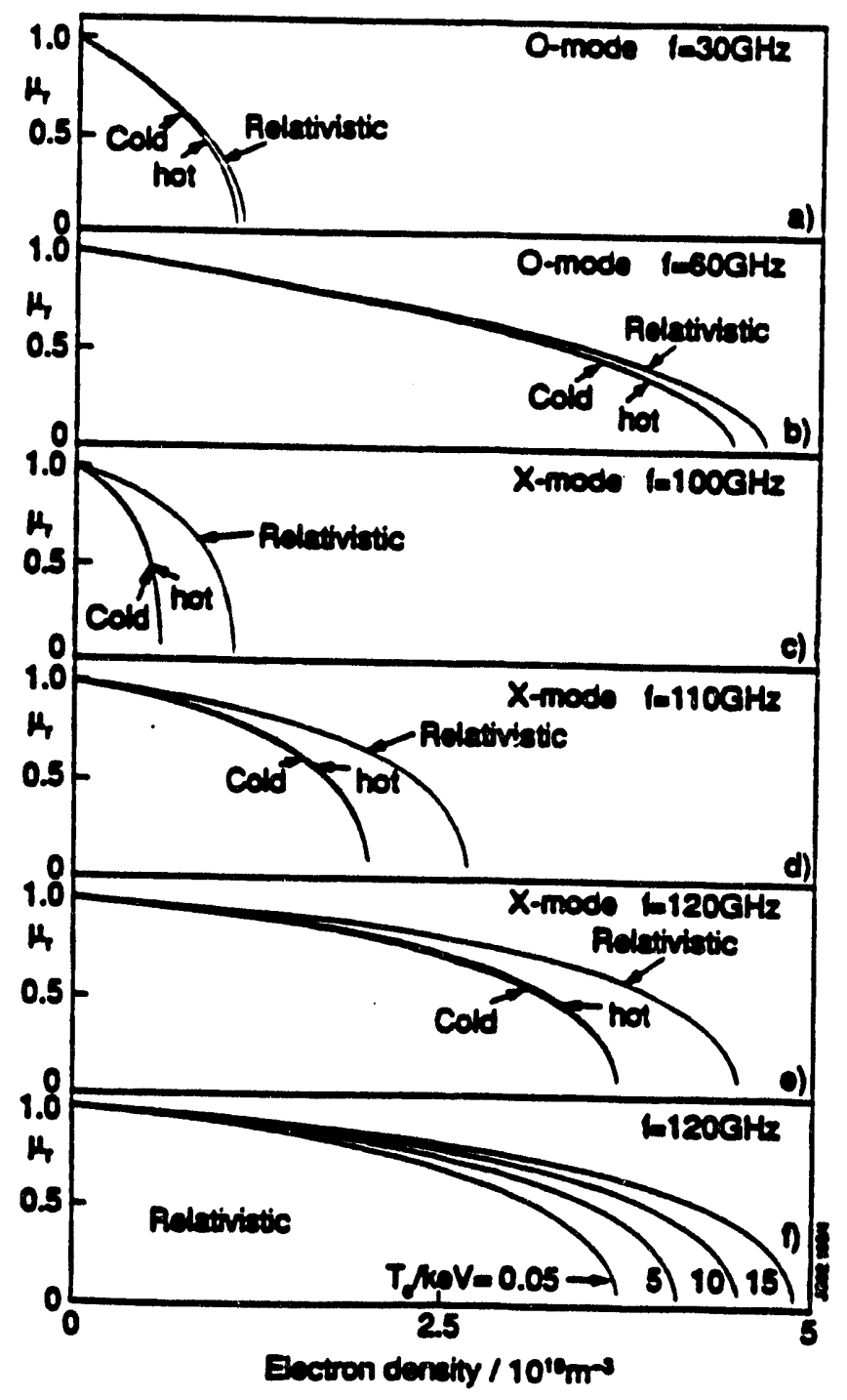

Figure 2.9: Relativistic effect on the index of refraction and the location of the cutoff using the weakly relativistic model. $\mu_{r}$ is the refractive index and $T_{e} \approx 10 \mathrm{KeV}$ for piots $a$ to $e$. 
assumed density profile. It can also be seen that the $\mathrm{X}$-mode underestimates the reconstructed density profile more than the O-mode. The plots in Figure 2.10 are as follows:

A. Plot "a)" shows the actual (assumed) density profile together with the reconstructed density profiles for different plasma temperatures using the O-mode wave.

B. Plot "b)" shows the actual (assumed) density profile together with the reconstructed density profiles for different plasma temperatures for an X-mode wave.

C. Plot "c)" is similar to the previous plot but the center density of the plasma and the magnetic field have been changed to $n_{e}\left(R_{0}\right) \approx 3.0 \times 10^{19} \frac{\text { part }}{\mathrm{m}^{3}}$ and $B_{0} \approx 3.4$ Teslas. An even bigger disagreement between the $\mathrm{O}$-mode and the $\mathrm{X}$-mode can be seen in these plots.

\subsubsection{Uncertainty in $\omega_{x}$}

The uncertainty in the measurement of the cutoff frequency can be calculated using the propagation of error formula given by Bevington ${ }^{66}$ as:

$$
\sigma_{\omega_{X}}^{2}=\sigma_{\omega_{e c}}^{2}\left(\frac{\partial \omega_{X}}{\partial \omega_{e c}}\right)^{2}+\sigma_{\omega_{p e}}^{2}\left(\frac{\partial \omega_{X}}{\partial \omega_{p e}}\right)^{2}
$$

where $\sigma_{\omega_{X}}$ is the uncertainty on the cutoff frequency, $\sigma_{\omega_{e c}}$ is the uncertainty on the electron cyclotron frequency, and $\sigma_{\omega_{p e}}$ is the uncertainty on the plasma electron frequency.

Using equation 2.67 for the upper cutoff (fast wave):

$$
\omega_{X}=\frac{\omega_{e c}}{2}+\left(\omega_{p e}^{2}+\left(\frac{\omega_{e c}}{2}\right)^{2}\right)^{\frac{1}{2}}
$$



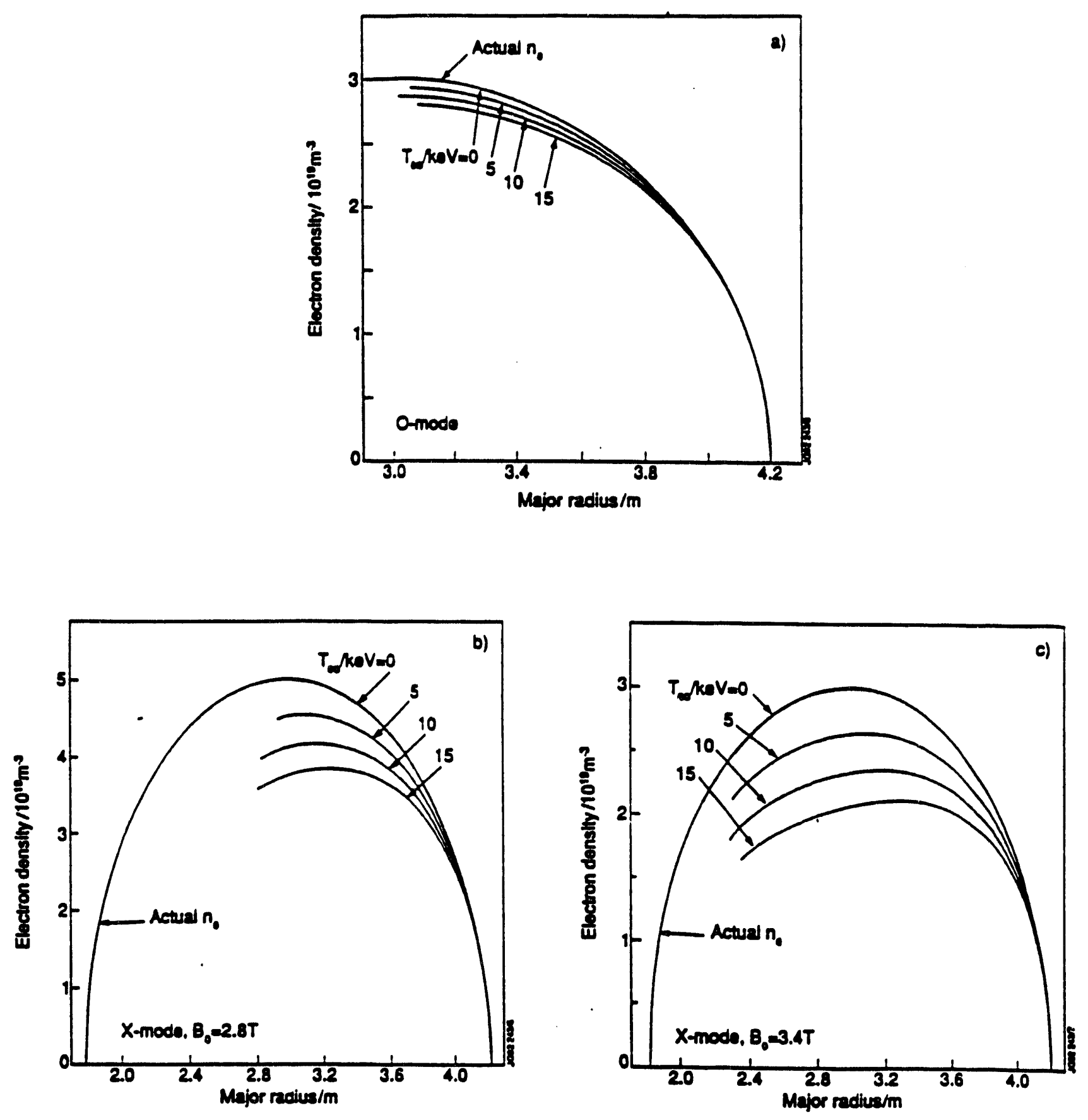

Figure 2.10: Comparison of the actual and reconstructed density profile using the weakly relativistic model. 
it follows that:

$$
\frac{\partial \omega_{X}}{\partial \omega_{e c}}=\frac{1}{2}+\frac{1}{4} \frac{\omega_{e c}}{\left(\omega_{p e}^{2}+\left(\frac{\omega_{e c}}{2}\right)^{2}\right)^{\frac{1}{2}}}
$$

and:

$$
\frac{\partial \omega_{X}}{\partial \omega_{p e}}=\frac{\omega_{p e}}{\left(\omega_{p e}^{2}+\left(\frac{\omega_{e c}}{2}\right)^{2}\right)^{\frac{1}{2}}}
$$

for the final result:

$$
\sigma_{\omega_{X}}^{2}=\sigma_{\omega_{e c}}^{2} \frac{1}{4}\left(1+\frac{\omega_{e c}}{2\left(\omega_{p e}^{2}+\left(\frac{\omega_{e c}}{2}\right)^{2}\right)^{\frac{1}{2}}}\right)^{2}+\sigma_{\omega_{p e}}^{2}\left(\frac{\omega_{p e}}{\left(\omega_{p e}^{2}+\left(\frac{\omega_{e c}}{2}\right)^{2}\right)^{\frac{1}{2}}}\right)^{2}
$$

where $\sigma_{\omega_{\text {ee }}}$ and $\sigma_{\omega_{\mathrm{pe}}}$ are the corresponding uncertainties in the electron cyclotron and plasma electron frequencies, respectively.

Next, this uncertainty can be expressed in terms of the uncertainties of the density, $\sigma_{n_{e}}$, and the magnetic field, $\sigma_{B}$, by using the equation:

$$
\omega_{p e}^{2}=\frac{q_{e}^{2}}{m_{e} \epsilon_{0}} n_{e}
$$

Using propagation of error gives:

$$
2 \omega_{p e} \sigma_{\omega_{p e}}=\frac{q_{e}^{2}}{m_{e} \epsilon_{0}} \sigma_{n_{e}}
$$

Dividing both sides by equation 2.213 yields:

$$
\frac{\sigma_{\omega_{p e}}}{\omega_{p e}}=\frac{1}{2} \frac{\sigma_{n_{e}}}{n_{e}}
$$

And for $\omega_{\text {ec }}$ :

$$
\begin{aligned}
\omega_{e c} & =\frac{q_{e} B}{m_{e}} \\
\sigma_{\omega_{e c}} & =\frac{q_{e}}{m_{e}} \sigma_{B} \\
\frac{\sigma_{\omega_{e c}}}{\omega_{e c}} & =\frac{\sigma_{B}}{B}
\end{aligned}
$$


for a final result of:

$$
\sigma_{\omega_{X}}^{2}=\frac{1}{4}\left(\frac{\sigma_{B}}{B}\right)^{2}\left(\omega_{e c}+\frac{\omega_{e c}^{2}}{\left(4 \omega_{p e}^{2}+\omega_{e c}^{2}\right)^{\frac{1}{2}}}\right)^{2}+\left(\frac{\sigma_{n_{e}}}{n_{e}}\right)^{2}\left(\begin{array}{c}
\omega_{p e}^{2} \\
\left(4 \omega_{p e}^{2}+\omega_{e c}^{2}\right)^{\frac{1}{2}}
\end{array}\right)^{2}
$$

which express the uncertainty of the frequency of the upper cutoff (fast wave) in terms of the uncertainties of the magnetic field and the density. This equation can be used to calculate the uncertainty on the reconstructed density profile if all the other parameters are known or are measurable from other diagnostics.

\subsection{Summary}

This chapter presents a brief history of the beginnings of reflectometry, a derivation of the cold plasma dielectric tensor, a description of the different reflectometry techniques, and a discussion of the complications and error sources that one can expect to find on this type of diagnostic.

This review of electromagnetic theory in plasma is essential to understanding plasmas and reflectometry. This gives the researcher the knowledge needed to understand the different phenomena that occur in a plasma and how any electromagnetic wave, including that of a reflectometer, propagates through the plasma. This chapter and the theory covered within it provide a set of formulae that can be used to calculate parameters such as the frequency band for the cutoffs, the relative position of the cutoffs, and the electron cyclotron resonances; the formulae necessary to calculate the theoretical phase to model the wave propagation and test the technique; and complications that can be associated with the measurement. 


\section{CHAPTER III}

\section{Ion Cyclotron Resonance Heating}

\subsection{ICRH vs Other Heating Methods}

In order to achieve fusion, the particles must have enough kinetic energy to overcome the Coulomb repulsive force during a collision. Such energy is provided in the form of heat, and the temperatures must be on the order of 10 to $20 \mathrm{KeV},{ }^{1,164}$ This is the range at which the cross section of the D-T reaction is the greatest. ${ }^{1,164}$ Among present day heat sources for plasmas there are:

1. Ohmic heating

2. Neutral Beam Injection (NBI)

3. Electron Cyclotron Resonance Heating (ECRH)

4. Adiabatic compression

5. Ion Cyclotron Resonance Heating (ICRH)

6. Fusion Alpha Heating

7. Lower Hybrid Heating (usually associated with current drive)

For ohmic heating the current that flows in the plasma transfers its energy to the particles through collisions and frictional effects, providing an intrinsic heating mechanism. Unfortunately, it can be shown that the ohmic power transfer saturates 
as the temperature increases (at around $3-4 \mathrm{KeV}$ ) ${ }^{1,164}$ thus, fusion probably can not depend entirely on ohmic heating to achieve fusion temperatures.

Neutral Beam Injection (NBI) has successfully provided additional heating and refueling capabilities, but it has been found that it degrades confinement of energy in toroidal devices. Neutral Beam Injection has a coupling in between heating and refueling of the plasma. For experimental purposes it makes it more complicated to study the independent effect of heating and refueling on the plasma. Electron Cyclotron Resonance Heating is starting to prove itself as a heating method and has a high dollars/Watt. ${ }^{155}$ Adiabatic compression does not work well in small volumes (requires large volumes), for heating electrons, and it does not have the positioning capabilities of RF schemes and NBI. ${ }^{164}$

Ion Cyclotron Resonance Heating (ICRH) methods have been used successfully to heat ions in fusion plasmas for the past 25 years, particularly in tokamaks ${ }^{143-146}$ and other ${ }^{9,147-150}$ fusion devices, over a wide range of confinement regimes, including $\mathrm{H}$-mode. ${ }^{151}$ In addition to provide a main source of auxiliary heating, several other functions are served by ICRH (RF) such as plama ionization, plasma temperature profile control, MagnetoHydroDynamic (MHD) stabilization and current drive. One major advantage of ICRH (RF) is that the position of the energy absorption layer is highly controllable as compared with other heating mechanisms such as Ohmic heating and Adiabatic compression. ${ }^{164}$ Thus, the position of the absorption layer can be controlled so as to change the current density and temperature profiles. ${ }^{1,164}$ These changes in the density and temperature profiles can be coordinated to suppress plasma instabilities (like sawteeth ${ }^{166,167}$ ), enhance confinement, and control plasma transport. ${ }^{184}$ Another major advantage of ICRH (RF) is in the area of current drive, since when a wave is sent into a tokamak at an angle to the major radius, it is capable of being absorbed in such a way as to set up a directional drift of electrons around the torus. ${ }^{164}$ This together with the control of the position 
of the absorption layer can be used to change the current profile. This is of extreme importance, since many important MagnetoHydroDynamic (MHD) instabilities in tokamaks are current driven. ${ }^{164,152-154}$ Thus, the ICRH (RF) power deposition profile might be controlled so that MHD instabilities can be avoided or suppressed.

At the moment NBI and ICRH (RF) are the methods most widely used to heat large tokamaks. For example, JET has $22 \mathrm{MW}$ of ICRH power while TFTR has $12.5 \mathrm{MW}^{156}$ of power available in the RF range. ${ }^{155,276}$ Due to its flexibility and relative low cost, ICRH is becoming a leading supplemental heat source for toroidal experiments.

\subsection{Theory}

\subsubsection{RF heating schemes}

The schemes that have been used for RF heating in tokamaks fall into four main frequency ranges; within which waves excited at the edge of the plasma may propagate into the central region of the plasma where they can be absorbed. The frequencies involved depend on many plasma parameters. The values given here are simply indicators of what is typical in present day fusion experiments. The lowest in frequency is the Alfvén wave, involving waves at a frequency of a few megahertz. This is well below the ion cyclotron frequency of a typical tokamak, so the waves involved are the shear and the compressional Alfvén waves. Next comes the ion cyclotron range of frequencies at a few tens of megahertz. This is presently the method most widely used to heat large tokamaks. Increasing the frequency to a few gigahertz leads to the Lower Hybrid range, which has been particularly successful for current drive experiments. Finally, electron cyclotron heating (also called UH resonance or electron cyclotron harmonic) occurs at frequencies of around $30 \mathrm{GHz}$ and upward. Development of this method has, to some extent, been held back by the 
difficulties of manufacturing high power sources at the necessary high frequencies.

\subsubsection{Multiple species dielectric tensor}

The theory of wave propagation and absorption in the ion cyclotron range of frequencies is the same one discussed in section 2.2 for the propagation of the reflectometer beam in the plasma. In this section this theory will be reviewed, which can explain more complicated heating mechanisms like ion-ion heating and minority species heating. The effects of multiple species (electrons and different kinds of ions) is included in the current density as:

$$
\vec{J}=\sum_{\bullet} n, q, \vec{v}_{\bullet}
$$

and the equation of motion of each species as:

$$
\frac{d \vec{v}_{e}}{d t}=\frac{q_{e}}{m_{c}}\left(\vec{E}+\vec{v}_{\iota} \times \vec{B}\right)
$$

where the subscript " $s$ " is used to designate the species (electrons and/or ions) and the other variables are the density, $n_{j} ;$ particle charge, $q_{q} ;$ velocity, $\vec{v}_{0}$ and mass, $m$, of the particular species. Following the procedure in section 2.2.2 and using the equations for the current density, equation 3.1, and the equation of motion, equation 3.2 , the velocity components for the particles of spicies " $s$ " are given by:

$$
\begin{aligned}
& v_{s x}=\frac{q_{s}\left(i \omega E_{x}-\epsilon_{o} \omega_{c o} E_{y}\right)}{m_{s}\left(\omega^{2}-\omega_{c o}^{2}\right)} \\
& v_{s y}=\frac{q_{0}\left(\epsilon_{s} \omega_{c,} E_{x}+i \omega E_{y}\right)}{m_{s}\left(\omega^{2}-\omega_{c s}^{2}\right)} \\
& v_{s z}=\frac{i q_{s}}{m_{s} \omega} E \text {. }
\end{aligned}
$$

or in matrix form as:

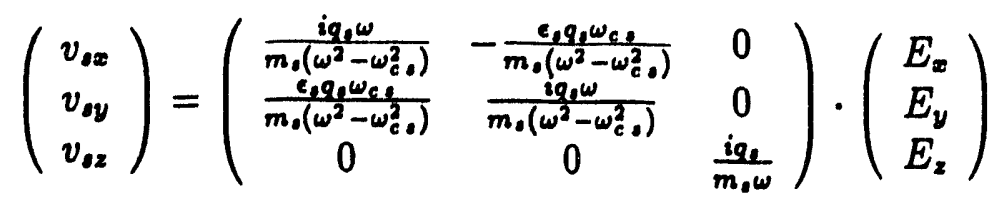


where $\omega$ is the frequency of the electromagnetic wave, $\omega_{c}$, is the corresponding cyclotron resonance frequency of species " $s$ ", and $\epsilon=\frac{q_{2}}{\left|q_{1}\right|}$.

Next, substituting the equation for the species velocities, equation 3.6 , into the current density, equation 3.1 , it can be obtained:

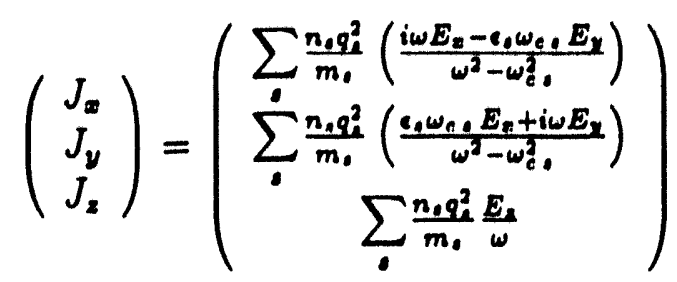

which can be substituted on the right hand side of the generalized Ampére's law as:

$$
\vec{J}-i \omega \epsilon_{0} \vec{E}=-i \omega \epsilon_{0} \bar{K}
$$

where the dielectric tensor is defined by:

$$
\overline{\mathrm{K}} \equiv\left(\begin{array}{ccc}
S & -i D & 0 \\
i D & S & 0 \\
0 & 0 & P
\end{array}\right)
$$

where:

$$
\begin{aligned}
& S \equiv \frac{1}{2}(R+L) \equiv 1-\sum_{,} \frac{\omega_{p,}^{2}}{\omega^{2}-\omega_{c,}^{2}} \\
& D \equiv \frac{1}{2}(R-L) \equiv \sum_{,} \frac{\epsilon_{0} \omega_{p,}^{2} \omega_{c 。}}{\omega\left(\omega^{2}-\omega_{c,}^{2}\right)} \\
& P \equiv 1-\sum \frac{\omega_{p s}^{2}}{\omega^{2}} \\
& R \equiv S+D \equiv 1-\sum \frac{\omega_{p}^{2}}{\omega\left(\omega+\epsilon_{\jmath} \omega_{c, s}\right)} \\
& L \equiv S-D \equiv 1-\sum \frac{\omega_{p \ell}^{2}}{\omega\left(\omega-\epsilon_{,} \omega_{c,}\right)} \\
& \omega_{p,}^{2} \equiv \frac{n_{0} q_{1}^{2}}{m_{,} \epsilon_{0}} \\
& \omega_{c s} \equiv \frac{q_{0} B}{m_{\imath}}
\end{aligned}
$$

These equations are very similar to the ones derived in the previous chapters, but they include the effect of multiple ion species, which describe other more subtle effects like ion-ion and minority heating in fusion plasma. 


\subsection{ICRH and Plasma Edge Interaction}

The plasma edge has always been of utmost importance to ICRH experimente, since it is the interface in between the antenna/launching structure and the bulk of the plasma interior. This interaction is becoming more important because of its role in understanding the plasma confinement and the control of the plasma composition. This interaction is bidirectional and sometimes even circular, in the sense that the ICRH influences the plasma edge directly or indirectly, through power deposition, ponderomotive effects, waves, sheath effects, induced electric field, and fluctuations. The plasma edge influences the ICRH by its effects on the antenna coupling and loading, see Figure $3.1,{ }^{187}$ impurity production, sputtering, etc. Most of the different phenomena and effects appeared to be specific to each machine and depend significantly on the different wall and limiter configurations, as well as the different plasma and heating conditions of the experiments, e.g., TFR, ${ }^{169,205}$ PLT, ${ }^{170,171,208,227}$ TORE SUPRA, ${ }^{172}$ MACROTOR, ${ }^{223}$ JIPPT-IIU, ${ }^{173}$ JFT-2M, ${ }^{209,174}$ URAGAN-3, ${ }^{175,176}$ Alcator C, ${ }^{177,178}$ ASDEX, ${ }^{179,224,229}$ TEXTOR, ${ }^{180-183}$ JET, ${ }^{235,184,185}$ JT-60, ${ }^{186}$ etc.

This section will describes the most important ICRH and plasma edge interactions; cause and effects (which are easily confused) will be presented.

\subsubsection{Pre-history}

For the ion cyclotron resonance frequency domain, the cold plasma dispersion relation has two branches that can be used to heat up the plasma. These are the slow wave (or also called the shear wave, or the torsional Alfvén wave, or the left hand wave), which propagates below the frequency of the ion cyclotron gyration frequency and can only penetrate to the central core of the medium density machines of the early times ${ }^{188}$ and the fast wave (or compressional wave, or hydro- 


\section{$n_{0}(A)$ FOR BPX (Roxto $\left.=279 \mathrm{~cm}\right)$}
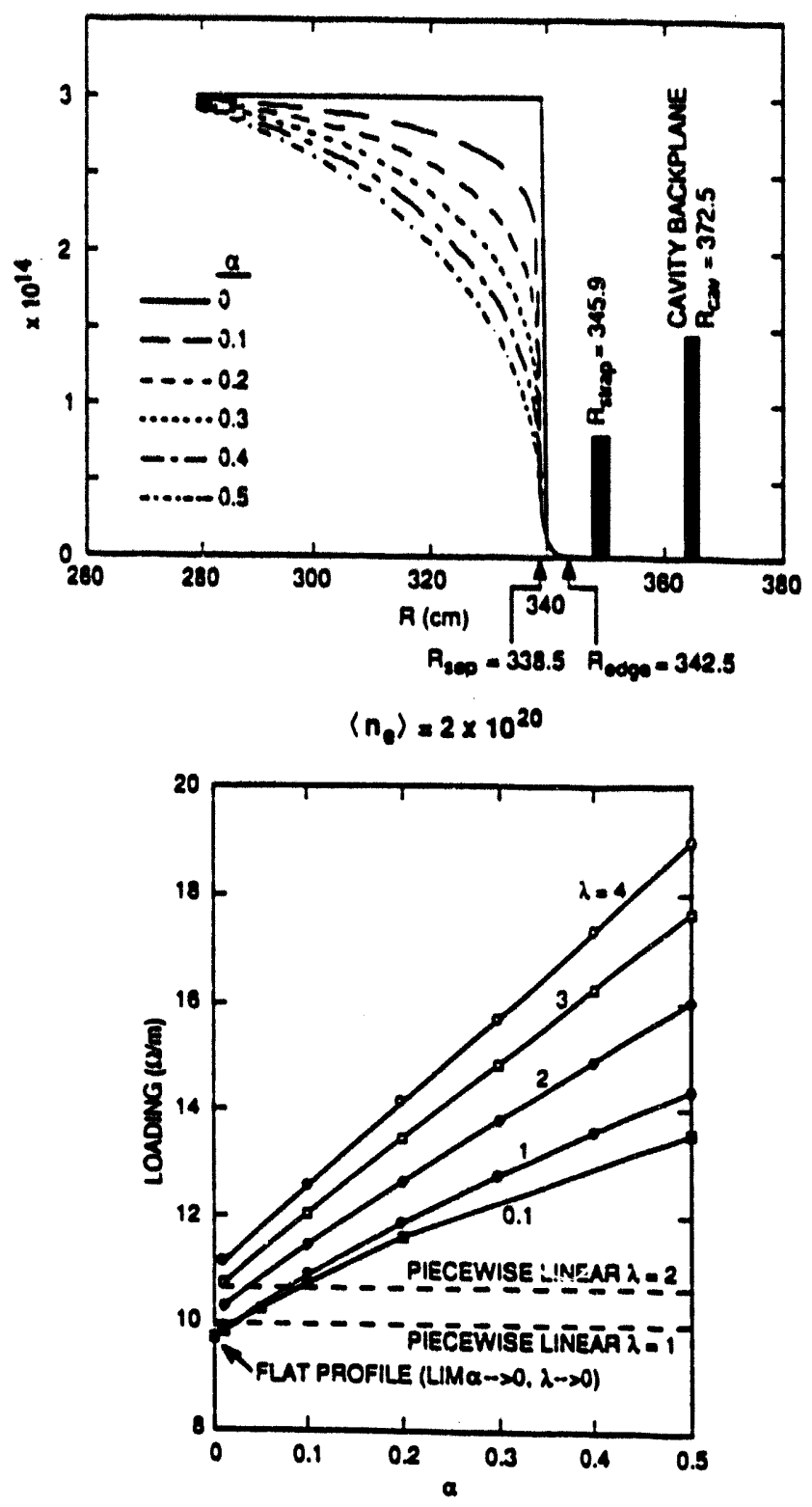

Figure 3.1: Typical parabolic density profiles of the form: $\left(1-\left(\frac{r}{a}\right)^{2}\right)^{\alpha}$ and antenna loading vs the profile shape exponent, $\alpha$, and the scrapeoff decay length, $\lambda$. (Batchelor, et al., Fusion Tech., Vol. 21 (1992) p. 1214) 
magnetic wave, or right hand wave), which propagates above a minimum density. ${ }^{180}$ The slow wave was initially used in early machines because it has a simple damping mechanism, since its electric field is circularly polarized in the ion gyration direction. During the early high power experiments in the C-stellarator, ${ }^{190}$ researchers observed a massive influx of impurities following the start of the ICRH power, accompanied by an anomalous loading of the coil, and they originally ascribed this phenomenon to the coupling to a mode called the "Mode $X$ ". The origin of the impurities was localized in the vicinity of the induction coil. This impurity influx was due to the sheath rectification effect. This occurred because the electric field component, $E_{z}$, parallel to the magnetic field, $B_{z}$, between the turns of the induction coil would penetrate the tenuous outer plasma layer. This caused the electrons to be accelerated in the $B_{2}$ direction, producing a spatial charge buildup, which in turn accelerated the ions into the wall. This effect has been observed 20 years earlier in linear plasmas discharges, ${ }^{101}$ and its mechanism has been explained by Butler. ${ }^{192}$ Short ICRH pulses had been used in the early machines to try to reduce the infiux of impurities. Later, the implementation of a Faraday shield screen shorted out these $E_{2}$ fields and reduced the impurity influx by an order of magnitude.

With the increasing success of the tokanak with higher densities and longer pulse times, the poor coupling of the slow wave at the higher densities became a concern, and the use of short RF pulses could not be used to avoid the impurity production problem. This was when the studies of the fast wave for ICRF heating became important. Experiments using the fast wave for ICRH were performed in tokamaks like TM-1 VCh in Moscow ${ }^{193-196}$ and in the ST-tokamak at Princeton. ${ }^{197-100}$ Even though the fast wave is circularly polarized in the direction of gyration of the electrons and the damping mechanism is not as simple as for the slow wave, three damping methods were found to absorb the wave in the plasma. These methods are: 
1. Using the second harmonic of the wave, since even in single species plasmas the wave has a component in the ion gyration direction.

2. The wave can be damped by the minority species of a two species plasma.

3. By launching the wave from the high field side of the plasma, the wave can reach the ion-ion hybrid resonance, at which it can be mode converted and damped.

\subsubsection{The impurity problem}

As solutions to the problems of launching and propa.gation of the wave were found, the problem of the effectiveness of heating became a concern. The negative effects of ICRH, and in particular the problem of increased impurity influx in the plasma, could no longer be ignored by shortening the ICRH pulse length. In order to pinpoint the possible causes of impurity influx, the specific machine dependent effects had to be avoided. For example, in TFR it took first the avoidance of ripple trapped ions to realize that the direct $R F$ interaction with the edge was the cause of the additional impurity problems. ${ }^{200}$ The influx of particles was strongly reduced by operating in the mode conversion regime rather than the minority heating regime. After a lot of debate, the cause for this specifically edge related increased impurity production was attributed to two possible causes:

1. ICRH and plasma edge interaction can increase impurity production.

2. ICRH could be changing the transport of impurities from the edge to the center of the plasma.

In both cases it was important to identify where and how the change was taking place. 
As higher ICRH power became possible, an increased influx of metallic impurities to the plasma center was observed. With the increase in plasma pulse length, the increase in impurities produced a decrease in central electron temperature of the plasma. The attention turned to changing the materiais of the antenna to avoid and/or reduce the production of impurities. ${ }^{201-205}$ Experiments with different materials for the antenna and Faraday shields reduced significantly the production of impurities, but could not reduce the high amounts of Nickel $(\mathrm{Ni})$ in the center of the plasma, thus pointing to the vacuum wall as a non-negligible source of impurities. ${ }^{205,206}$ Measurements of the density and temperature at the scrapeoff layer showed an increase in the density and temperature for both the ions and the electrons. These were seen as the main reasons for the increased interaction with the wall. ${ }^{205,206}$ After a lot of work, the mechanism was thought to be related to direct interaction between the RF and the plasma edge. ${ }^{205,206}$

Careful planning and selection of the ICRH antenna material has been shown to reduce significantly the production of impurities in the plasma. An example of this is the PLT tokamak at Princeton, in which the use of Carbon limiters and strong Titanium gettering to reduce Oxygen content in the plasma helped to reduce impurity production. This allowed for the study of other sources of impurities, such as the enhanced influx of particles from the surface of the plasma due to the RF interaction. ${ }^{207}$ Detailed analysis of the changes in density at the edge were performed using a low-energy neutral spectrometer. A large increase of the low-energy neutral hydrogen efflux during ICRF heating was observed. ${ }^{207}$ The edge density increased by an order of magnitude in some cases. Thus, it was concluded that the application of ICRH increases the radial transpor $\hat{\imath}$, thereby increasing the edge density and the plasma interaction with the vacuum wall. ${ }^{207}$ Further experiments with the position of the resonance layer and the minority concentration pointed out a correlation between the density increase and the wave absorption. ${ }^{208}$ That is, the increase in 
plasma edge density was a minimum when the wave absorption was a maximum.

Other effects seem to be responsible for the impurity production in other machines. In JFT-2M, sputtering induced by ions accelerated by the electric field near the antenna was proposed as an explanation for the impurity production. ${ }^{209-211}$ The effect of this mechanism could be controlled in two possible ways: the phasing of the antennas and the choice of the material in the antenna neighborhood. The operation of the antennas out of phase (i.e., the electric field of the radiating elements of the antennas are $\pi$ radians out of phase) reduced the levels of metallic impurities significantly, ${ }^{212,213}$ while the installation of carbon blocks on the wall in the vicinity of the antennas greatly reduced the difference between the in phase and out of phase operation, ${ }^{214}$ supporting the theoretical prediction that the electric field near the antenna played a dominant role in inducing the metallic impurity release. Reduction of metallic impurities has been observed in TFTR when the ICRH antennas are operating in an out-of-phase mode. ${ }^{243}$

\subsubsection{Antenna phasing}

The influence of antenna phasing is one of the most important phenomena studied in ICRH plasmas. In almost all experiments with two or more radiating elements (antennas), there is a distinct advantage to driving the antennas in an out of phase configuration (dipole configuration) as opposed to drive the antennas in an in phase configuration (monopole configuration). Heating efficiencies are $20-30 \%$ lower, and both low- and high- $\mathrm{Z}$ impurities are 30-50\% higher when the antennas are used in the in phase (monopole) configuration. ${ }^{244}$ Local arcing of the antennas has been observed only during the operation of the in phase (monopole) configuration. 


\subsubsection{The sheath rectification effect}

The sheath rectification effect was first seen 20 years ago in linear plasma discharges. ${ }^{218,217}$ This effect works by creating a potential in the direction of the field lines in front of the antenna. Where these field lines intercept the Faraday screen, the RF potential will be rectified, and the ions around these field lines will be accelerated into the screen, creating impurity ions off the Faraday screen that will migrate to the plasma. Calculations have shown that under these conditions, the electric field would be enhanced in the immediate vicinity of the Faraday screen, resulting in an increased induced potential. ${ }^{215}$ Numerical simulations indicated that field lines in the antenna neighborhood can acquire an $\mathrm{RF}$ potential, even if not by direct induction, so that the sheath rectification effect could be retained as the fundamental driving mechanism for the impurity generation. ${ }^{216,217}$

Perkins ${ }^{218,219}$ and $\mathrm{Myra}^{220}$ have proposed a mechanism in which parallel electric fields associated with the antenna can give rise to a significant increase of the energy of the ions impacting the Faraday shield. As a result of the parallel electric fields, the sputtering yield is much higher than the one expected for ions that fall through the normal Bohm sheath. The source of the parallel electric fields may come from the RF sheath (image charges) that are produced from the misalignment between the Faraday shield elements and the magnetic field lines. ${ }^{218,219}$

Caughman ${ }^{21,222}$ and others ${ }^{23,224}$ have found evidence of increased electron temperature, plasma potential and ion energy near the Faraday shield. In his experiment, the time varying floating potential followed the magnetic field pattern of the antenna, indicating that the electromagnetic field is responsible for the potential formation. The increase in the ion energy can be at least partially explained by an increase in the sheath potential, which in turn is caused by an increase in the electron temperature. These high energy ions that are produced in front of the ICRH antenna contribute to the sputtering and the production of impurities into 
the plasma. ${ }^{225}$

\subsubsection{Self sputtering and avalanche}

The different reactions of the different materials to the antenna induced impurity and the sheath rectification effect was resolved by a theoretical model proposed for JET. ${ }^{232}$ The sputtered impurities produced by the sheath rectification effect could be re-ionized and accelerated back into the wall, producing more impurities; hence, the name self-sputtering. This process could become unstable and self-amplifying, producing an avalanche effect in the generation of sputtering ions.

\subsubsection{Increased diffusion at the edge}

Radial electric fields were observed in early fusion experiments. It has been suggested that these radial electric fields could create flows in the plasma. ${ }^{227-231}$ This will create convective flow cells that will change the edge transport. Convective cells flow was analyzed by D'Ippolito ${ }^{232}$ and it was found that radial electric fields could lead to the required RF enhanced diffusion at the edge. Direct evidence of convective cell was found by measurements of the electric field in the edge and by edge fluctuation measurements. ${ }^{233,234}$ Edge electric fields are observed in $\mathrm{H}$-mode transition layers in DIII-D. ${ }^{110}$

Associated with these effects are changes in the edge scrapeoff layer. This typically includes an increase in the electron temperature, increase in the edge density and an increase of the scrapeoff lengths. ${ }^{235,236}$ This is significant, since the last of these suggests that an RF-induced edge transport mechanism is in effect. Work by Greene, ${ }^{237}$ Taylor, ${ }^{227}$ and Van Nieuwenhove ${ }^{238}$ demonstrated the formation of D.C. edge potentials considerably larger than the ones expected from the normal Bohm sheaths. 


\subsubsection{Quiver induced losses}

Because of the high electromagnetic fields at the interface between the plasma and the antenna, the quiver motion of particles (the velocity component associated with the effect of the RF fields as the particles pass by the antenna) passing near the antenna can drift into the Faraday shield and wall, thus producing direct losses of particle and inducing sputtering into the plasma. It was found ${ }^{239}$ that the quiver motion of ions can lead to particle energies considerably in excess of thermal energies, and that the RF can induce ion transport into the Faraday shield. The Faraday shield small scale non-uniformity produces a significant perpendicular ponderomotive force on the ions, leading to a diffusion coefficient of about two times Bohm.

\subsubsection{Ponderomotive effects}

Lehrman ${ }^{239}$ has done an analysis of the trajectories for the electrons and ions due to the antenna near fields. It was found that the electrons were mainly unperturbed, while the ions experience a strong perpendicular ponderomotive drift force due to the large evanescent fields in the vicinity of the Faraday shield. The flow in the vicinity of the Faraday shield is alternatively radially inward and outward as a consequence of the local electric fields in the shield gaps. Because ions reaching the Faraday shields are lost, there is an outward flux of particles.

\subsection{Antennas in Fusion Experiments}

There are many kinds of antennas currently in use in fusion plasmas. ${ }^{240}$ They have different characteristics with respect to coupling to the plasma, resistance to the plasma environment, power delivered, voltage limits, frequency domain, etc. 
M. Kwon ${ }^{253}$ made some measurements of the coupling of these parameters to the Faraday shields on different ICRH antennas. The most common antennas in use today are: ${ }^{240-251}$

1. The simple (short) loop is characterized by a total length that is short compared to the free space wavelength of the wave. It can be represented by an inductor and a resistor in series, as shown in Figure 3.2. It is not matched at the feed point.

2. A natural extension of the short loop is the long loop antenna shown in Figure 3.2 and used on Alcator-C, ${ }^{240,241,254,255}$ Figure 3.3. Because the dimensions are large compared to the wavelength, the circuit representation must be a distributed circuit. Like its relative the short loop antenna, it requires matching.

3. The cavity antenna shown in Figure 3.4 follows. This kind of antenna is used in the DIII-D experiment. ${ }^{165,240}$ It uses a tuning capacitor and corresponding feed point to provide a match at the feed point for a certain load.

4. The Resonant Double Loop (RDL) and the Asymmetric Resonant Double Loop (ARDL), shown in Figure 3.5, which are both matched at the feed point over both frequency and load ranges by means of two tuning capacitors. RDL antennas have been used successfully in TFTR, ${ }^{241}$ TORE-SUPRA ${ }^{241,251,252}$ and elsewhere. ${ }^{168}$ Their particular details can be found in the references. ${ }^{240,241}$

5. Finally, the U-slot antenna, shown in Figure $3.6,{ }^{256}$ which is tunable over load and frequency ranges by two tuning capacitors.

A list of experiments and corresponding operational parameters is shown in Table 3.1. ${ }^{157-163}$ 
CHAPTER 3: Ion Cyclotron Resonance Heating

ONNL-OWO 83.23002N FEO

LOOP ANTENNA

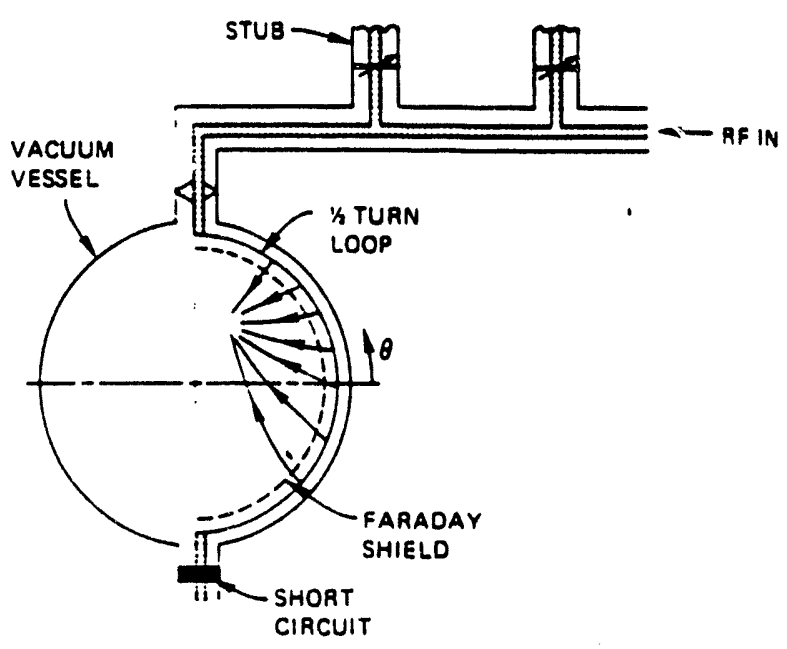

SIMPLE LOOP CIRCUIT

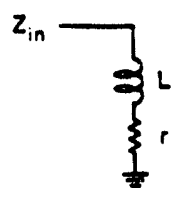

LONG LOOP CIRCUIT

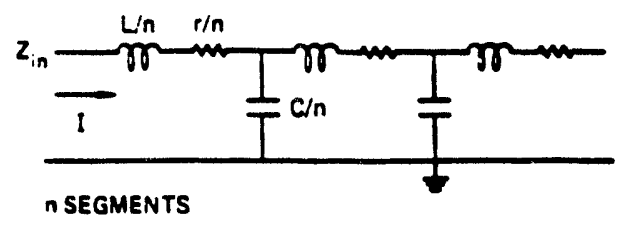

Figure 3.2: Loop antenna and circuit representation of both, the short and long loop versions. 


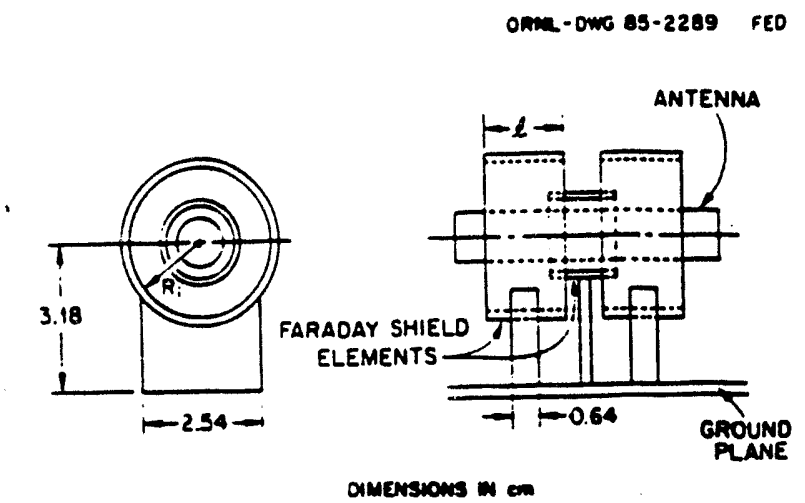

Figure 3.3: Long loop antenna used in Alcator-C.

\begin{tabular}{|c|c|c|c|c|c|c|c|}
\hline Exp. & $\begin{array}{l}\mathrm{R} \\
\mathrm{m}\end{array}$ & & $\begin{array}{l}\bar{n}_{e} \\
10^{18} m^{-3} \\
\end{array}$ & $\begin{array}{l}\mathrm{f} \\
\mathrm{MHz}\end{array}$ & $\begin{array}{l}P_{r f} \\
M W\end{array}$ & $\begin{array}{l}t_{r f} \\
\text { sec }\end{array}$ & $\begin{array}{l}\text { Heating } \\
\text { scheme }\end{array}$ \\
\hline TFTR & 2.6 & 0.96 & $\leq 5.0$ & 43,64 & 12.5 & 3 & $\mathrm{D}(\mathrm{H})$ ICRH \\
\hline JET & 3 & 1.2 & $\leq 4$ & $\begin{array}{l}25-55 \\
48 \\
\end{array}$ & 20 & $\leq 8$ & $\begin{array}{l}\mathrm{D}(\mathrm{H}), \mathrm{D}\left({ }^{3} \mathrm{He}\right) \\
\mathrm{ICRH}+\mathrm{NI}\end{array}$ \\
\hline Tore Supra & 2.35 & 0.78 & $\leq 5.0$ & $35-80$ & 4.2 & 2.0 & ICRH \\
\hline DIII-D & 1.67 & 0.67 & $\leq 14$ & $30-60$ & 2 & 5 & $\mathrm{D}(\mathrm{H}) \mathrm{ICRH}$ \\
\hline JT-60U & 3.3 & 1.0 & $\leq 4$ & $110-130$ & $\leq 2.5$ & $\leq 2$ & $2 \omega_{C B}$ in $\mathrm{H}$ \\
\hline TEXTOR & 1.75 & 0.46 & $\leq 6$ & $25-29$ & 2.2 & $\leq 2$ & $\mathrm{MC}(\mathrm{D}(\mathrm{H}))$ \\
\hline ASDEX & 1.67 & 0.40 & $\leq 9$ & $\begin{array}{l}30-115 \\
33.5 / 67\end{array}$ & 2.2 & $\leq 2$ & $\begin{array}{l}\mathrm{D}(\mathrm{H}), 2 \omega_{C H} \\
\mathrm{H} \text { and } \mathrm{H} / \mathrm{D} \text {, } \\
\mathrm{ICRH}+\mathrm{NI}\end{array}$ \\
\hline JFT-2M & 1.31 & 0.42 & $\leq 5$ & $15 . / 17$. & 2 & 0.3 & $\begin{array}{l}\mathrm{MC}(\mathrm{D}(\mathrm{H})) \\
\mathrm{ICRH}+\mathrm{NI}\end{array}$ \\
\hline $\begin{array}{l}\text { JIPPT } \\
\text {-IIU }\end{array}$ & 0.91 & 0.23 & $\leq 7$ & 40 & 2.2 & $\leq 0.1$ & $\begin{array}{l}\mathrm{MC}(\mathrm{D}(\mathrm{H})) \\
\mathrm{IBW}\end{array}$ \\
\hline ALC-C & 0.64 & 0.13 & $\leq 20$ & 180 & 0.5 & 0.07 & $\begin{array}{l}\mathrm{D}(\mathrm{H}), 2 \omega_{C H} \\
\mathrm{IBW}\end{array}$ \\
\hline HEL-E & 2 & 0.2 & $\leq 2.5$ & 26.7 & 1.5 & $\leq 0.2$ & $\begin{array}{l}\mathrm{MC}(\mathrm{D}(\mathrm{H})) \\
\mathrm{IBW}\end{array}$ \\
\hline
\end{tabular}

Table 3.1: RF experiments list. 


\section{CAVITY ANTENNA}

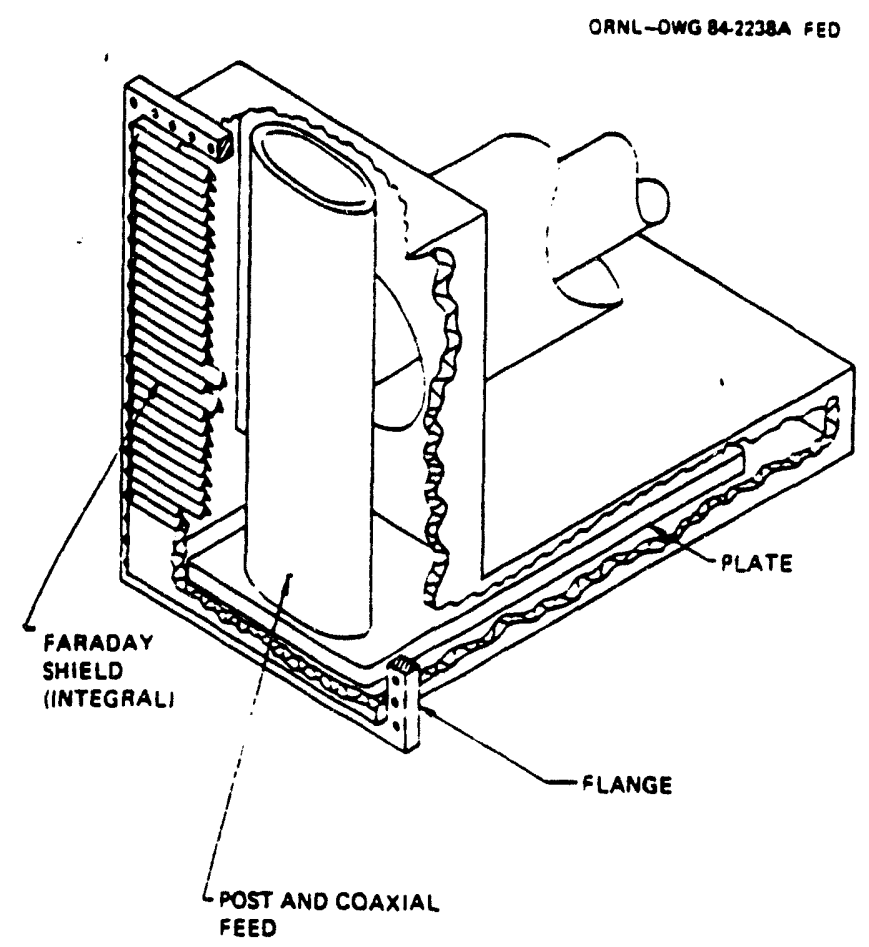

CAVITY CIRCUIT

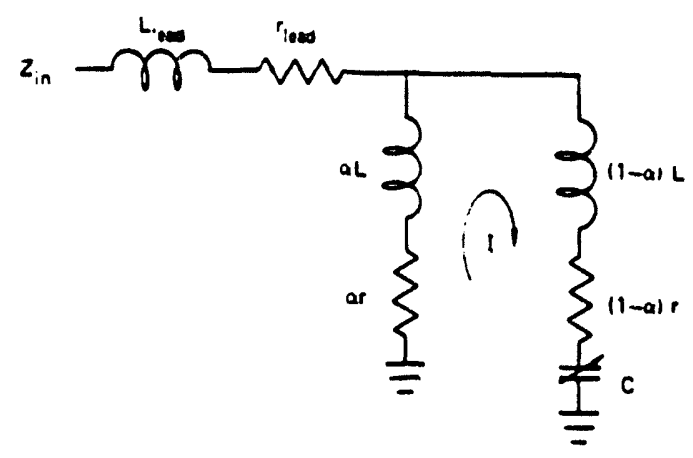

Figure 3.4: Typical cavity antenna and its equivalent circuit. 


\section{RESONANT DOUBLE LOOP (SIMPLE OR ASYMMETRIC)}

ORNL-E.VG 85.2276A FED

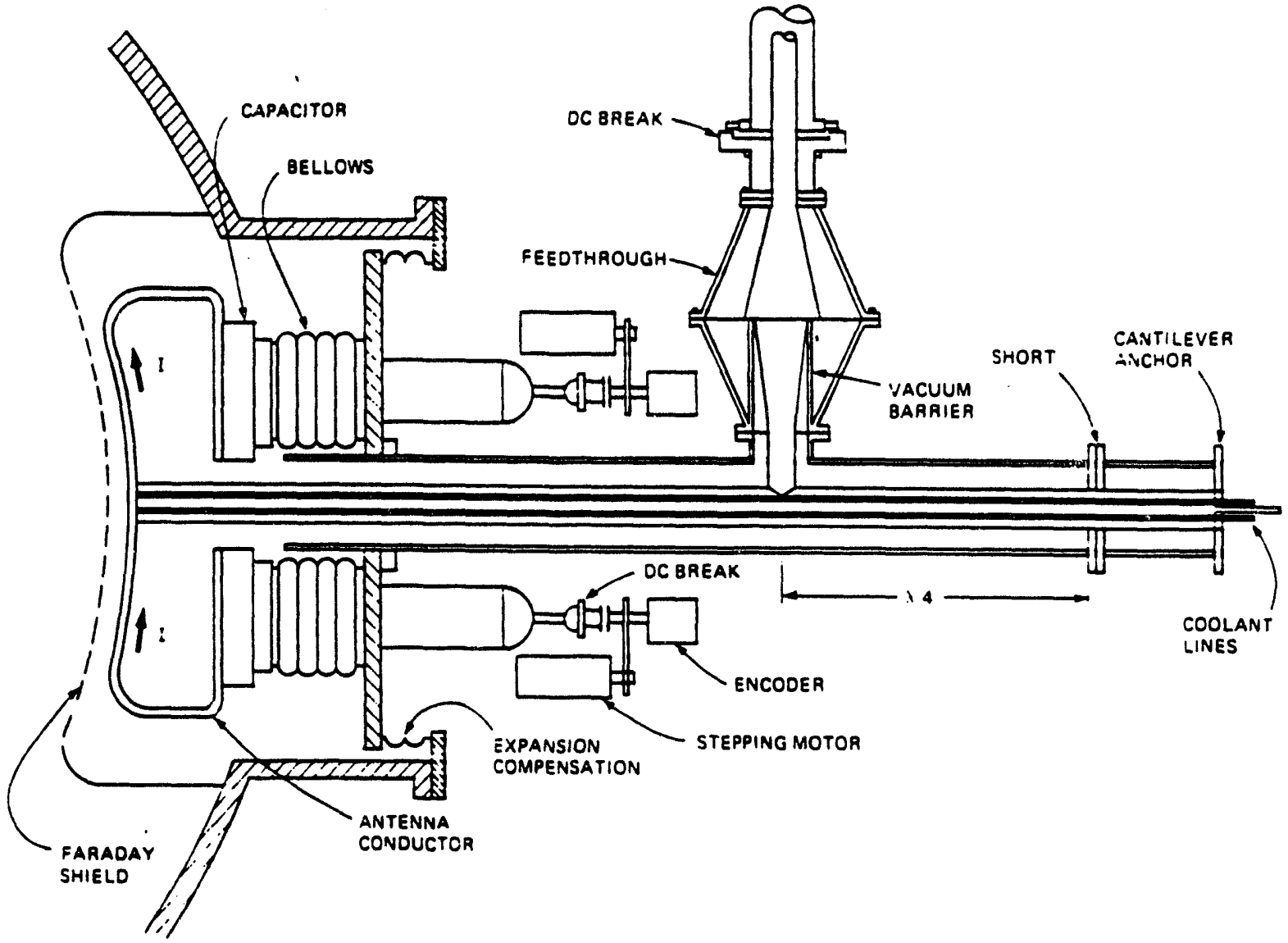

RDL AND ARDL CIRCUIT

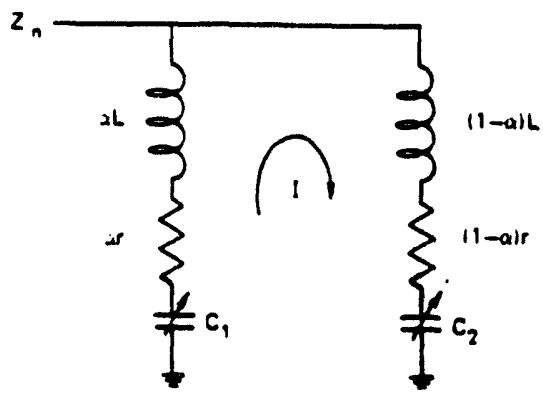

Figure 3.5: Resonant Double Loop antenna and its equivalent circuit. The symmetric version has $\alpha=0.5$. 


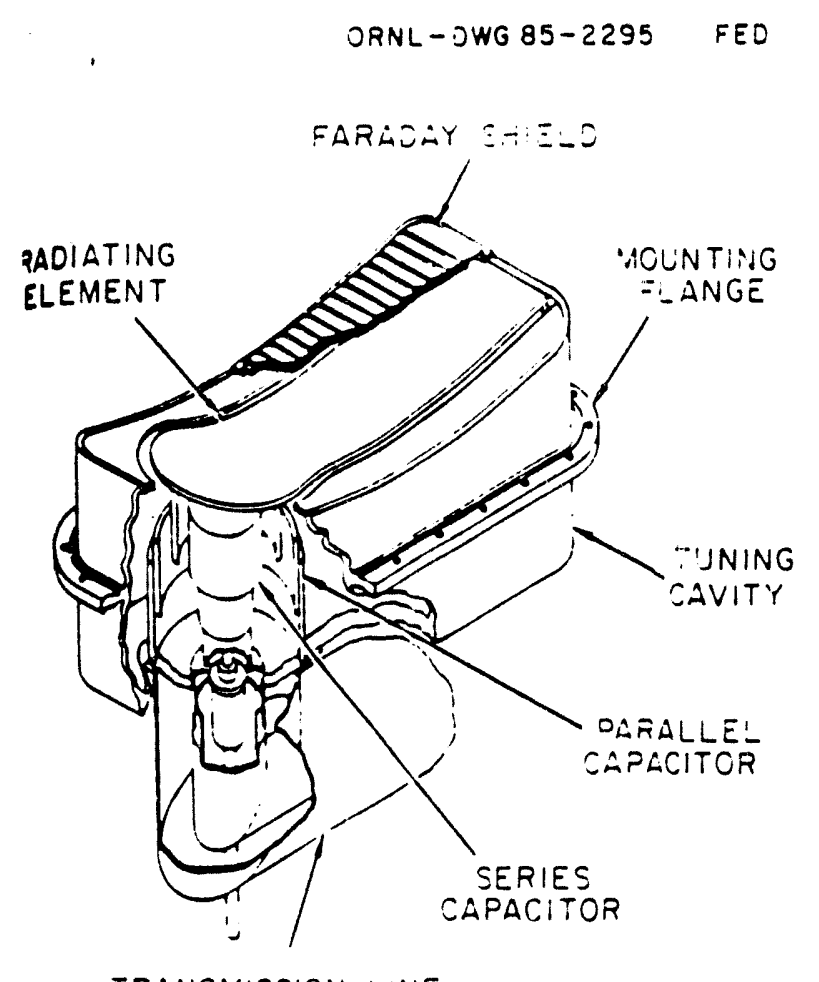

TRANSMISSION LINE

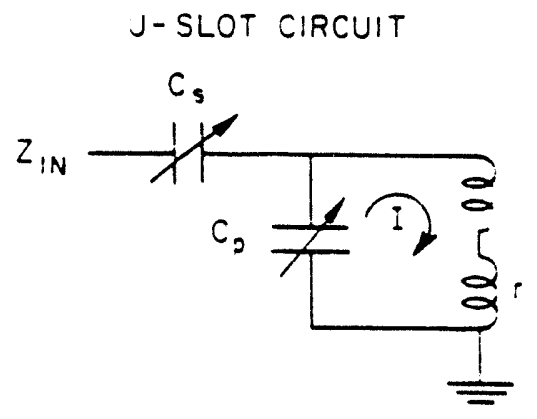

Figure 3.6: The U-slot antenna, as proposed by McDonnell Douglas Astronautics Company and its equivalent circuit. 


\subsection{TFTR Antenna}

TFTR's ICRF antenna has been the subject of much work. Some of the references are: J. R. Wilson, ${ }^{242}$ D. J. Hoffman, ${ }^{240,241}$ D. T. Taylor, ${ }^{251}$ J. F. King, ${ }^{250}$ J. E. Stevens, ${ }^{243,244}$ G. L. Chen, ${ }^{245}$ F. W. Baity, ${ }^{246}$ W. L. Gardner ${ }^{247}$ and others. ${ }^{248,249}$

Table 3.2 lists the antenna's main design parameters. ${ }^{240}$ Figure 3.7 and Figure 4.5 shows layouts of an antenna used on TFTR. While Figure 3.8 shows the cavity box of the antenna in more detail. The antenna consisted of a pair of resonant loops, separated by a solid dividing septum, in a single movable housing designed to fit through a horizontal midplane port of approximately $60 \mathrm{~cm}$ by $70-90 \mathrm{~cm}$. Each loop was designed to launch up to $2 \mathrm{MW}$ of power, for a total of $4 \mathrm{MW}$ per port in two bands from $40-80 \mathrm{MHz}$. A pair of capacitors was used to tune the antenna and match its impedance to $50 \Omega$. The tuning capacitors were designed at ORNL to minimize electric field at the expense of tuning range. A single Faraday shield protects the antennas. It consist of two tiers of actively cooled Inconel tubes, the front tier was covered with semicircular g,raphite sleeves to minimize the introduction of high- $\mathrm{Z}$ impurities into the plasma from the Faraday shield. The TFTR antenna was designed for 2 seconds pulses.

\subsection{Summary}

In this chapter the basis for RF wave heating has been reviewed, together with the effects of the RF power on the plasma edge. Some of the RF effects include: impurity generation, sheath rectification, self sputtering and avalanche, quiver motion, ponderomotive effects and antenna phasing. A presentation of the typical heating antennas used in fusion experiments was given, along with a description of TFTR's ICRF antenna. The importance of the effect of the edge density pro- 


\begin{tabular}{|l|l|}
\hline Description & TFTR \\
\hline Power into the port & $4 \mathrm{MW}$ \\
Power per current strap & $2 \mathrm{MW}$ \\
Port dimension & $60 \times 90 \mathrm{~cm} \times \mathrm{cm}$ \\
Total frequency range & $40-80 \mathrm{MHz}$ \\
Frequenc' range, first band & $40-60 \mathrm{MHz}$ \\
RF pulse length & $2 \mathrm{sec}$ \\
Capacitor designer & ORNL \\
Capacitor voltage peak & $50 \mathrm{kV}$ \\
Capacitor current (rms) & $800 \mathrm{Amp}$ \\
Capacitor electric field & $43 \mathrm{kV} / \mathrm{cm}$ \\
Antenna electric field & $23 \mathrm{kV} / \mathrm{cm}^{2}$ \\
Faraday shields losses & $100 \mathrm{~W} / \mathrm{cm}^{2}$ \\
Antenna power density & $1160 \mathrm{~W} / \mathrm{cm}^{2}$ \\
Antenna motion range & $11 \mathrm{~cm}$ \\
\hline
\end{tabular}

Table 3.2: TFTR antenna design parameters.

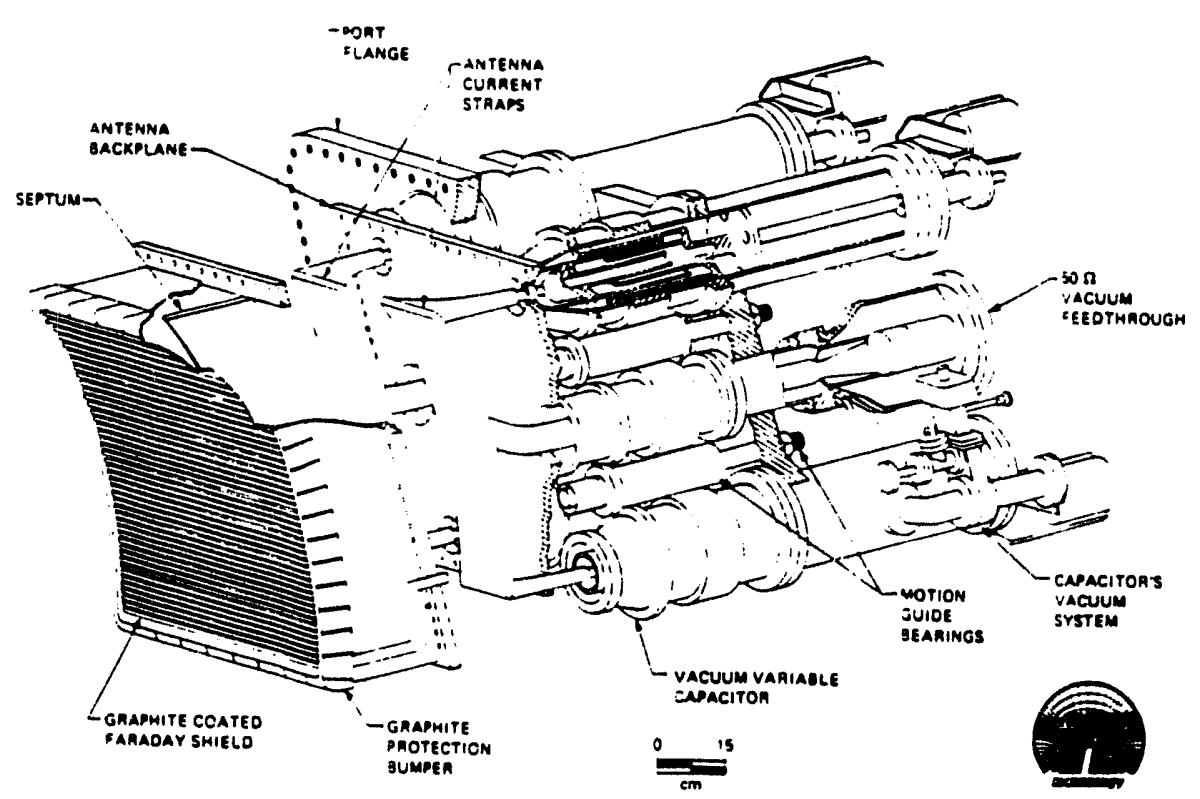

Figure 3.7: TFTR antenna layout. 


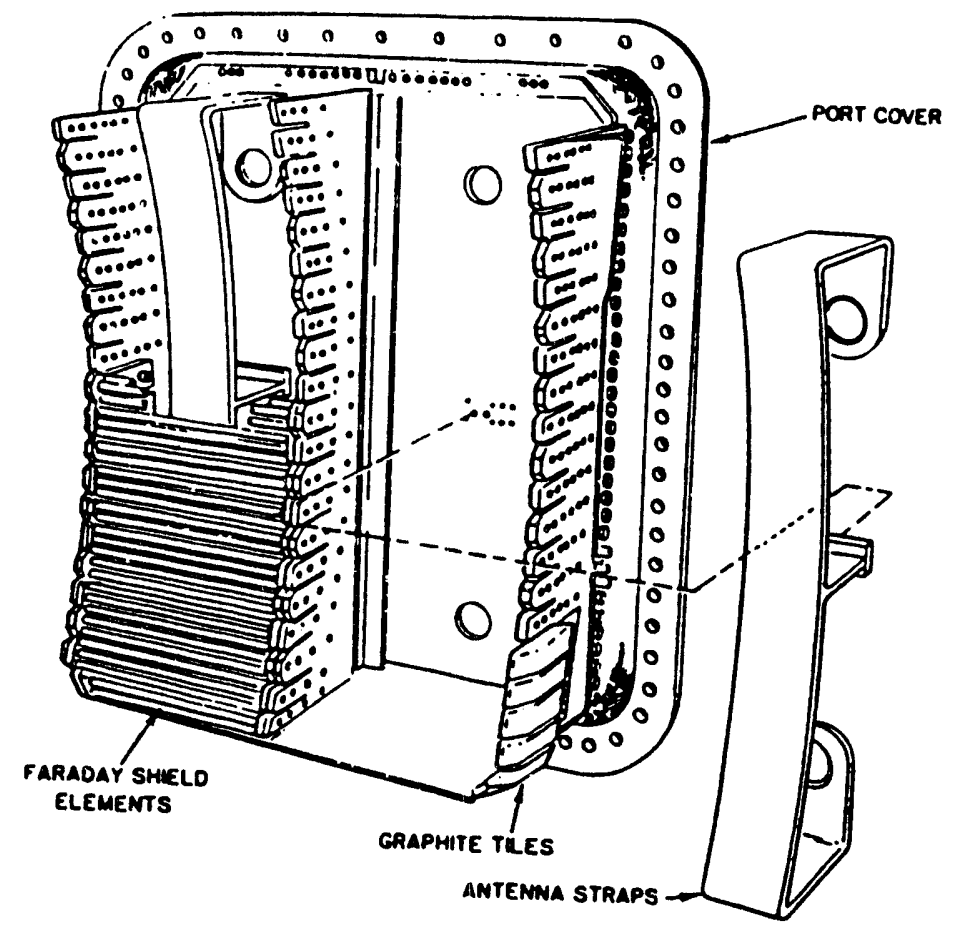

Figure 3.8: TFTR antenna cavity box. Most of the Faraday screen elements have been removed to show the current strap housing and the straps relative size. 
file on the ICRH antenna coupling and loading was presented in section 3.3 and in Figure 3.1, emphasizing the importance of the measurement of the plasma edge density in front of the ICRH antenna for calculations and comparisons between the theoretical and calculated loading and coupling of the plasma. 


\section{CHAPTER IV}

\section{TFTR REFlectometer System}

The interest of the new TFTR reflectometer is to study the edge density profile in front of the ICRH antenna, from within the high power environment of the antenna. Thus, making a direct measurement of the changes of $\mathrm{RF}$ power on the plasma edge. This changes on the edge density profile can change the coupling and loading of the antenna, which will change the RF power deposition on the plasma.

The reflectometer system proposed for TFTR has been discussed in detail in the references ${ }^{39,40,274,275}$ and it is shown in Figure $4.1^{39,40,274,275}$ with some characteristics of the main components. The system has one source at the range of $8 \mathrm{GHz}$ to $12.4 \mathrm{GHz}$ which provides the sweepable part of the probing wave and multiple sources at the frequency of about $7 \mathrm{GHz}$ which serve as up-converters for the probing wave. The different frequency multipliers before the launching antenna are to increase the frequency of the probing wave to the frequency range of the plasma reflection layers.

\subsection{Development}

Because of the TFTR requirements and working conditions, the new reflectometer could not be a copy of any pre-existent reflectometer system. These conditions include, but not only: 


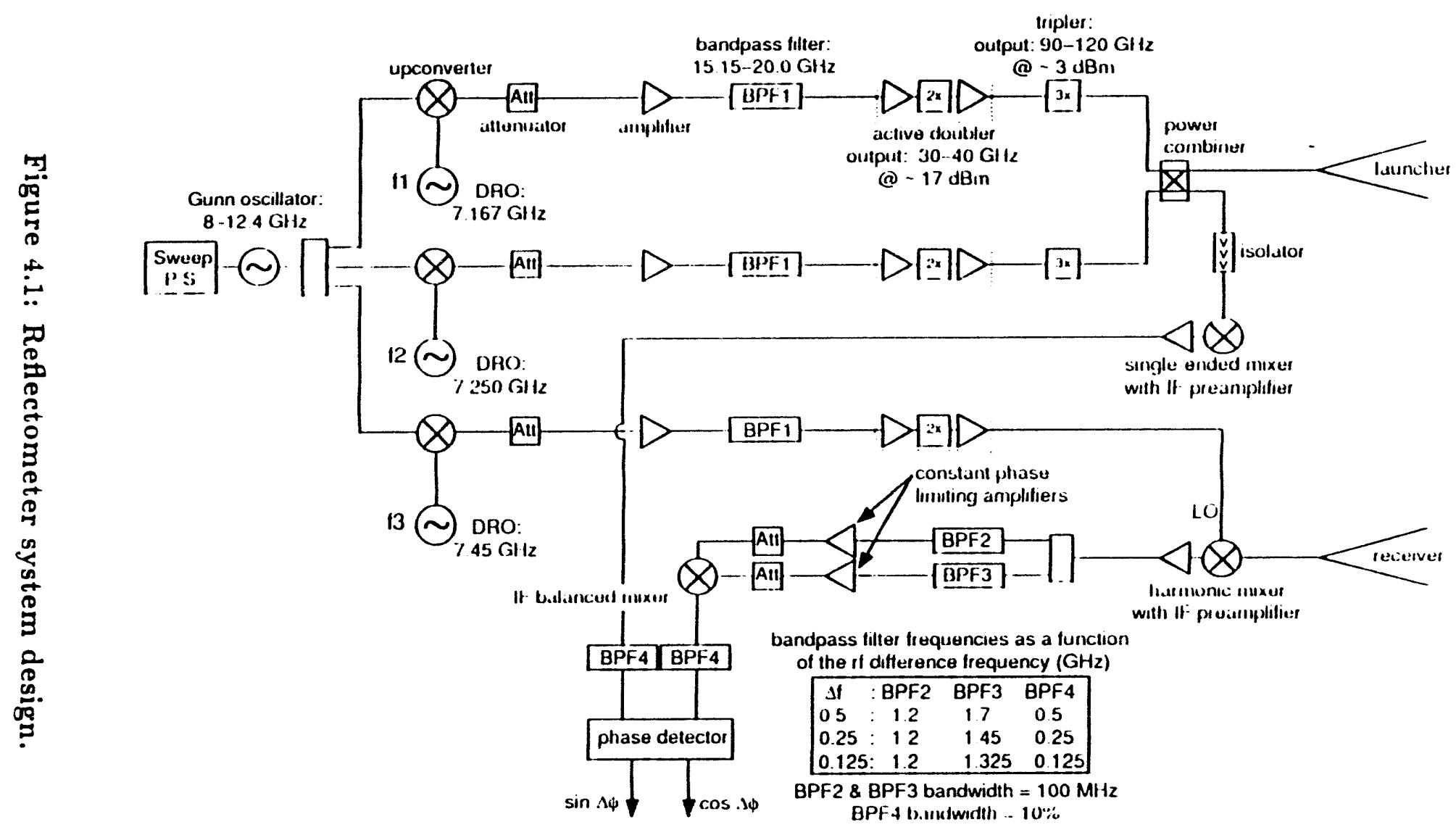


1. Inaccessibility: Because of the D-T phase of TFTR, access to the tokamak will be difficult and infrequent. Placement of the reflectometer electronics outside the TFTR enclosure with the corresponding $100+$ feet of waveguide poses other design problems.

2. Long distances: The long waveguide lenghts makes phase detection quite difficult for conventional reflectometers. For this reason, the phase difference technique was utilized, to minimize the fringe skipping associated with density fluctuations and to reduce the fringe count. This in itself brings up the problem of more complicated electronics.

3. Frequency range: Edge probing using conventional $\mathrm{O}$-mode operating reflectometers would require a very wide range of frequency, which is not only limited by the waveguide band limits, but would involve relative long wavelengths at the edge, which degrades the spatial resolution. This is why the X-mode of propagation was chosen for the probing wave. The X-mode reflectometer can start the frequency sweep at the local electron cyclotron resonance frequency on the edge, which is around $90 \mathrm{GHz}$.

4. New invesion technique: $\mathrm{X}$-mode data inversion techniques are more complicated but can provide all the necessary information once understood and developed.

5. Very high frequency electronic components: Due to the high magnetic field operation environment, high frequency waves (on the order of $100 \mathrm{GHz}$ ) are needed to probe the plasma.

TFTR can produce a wide range of plasma scenarios for the study of plasma transport, ICRH heating and other important parameters relevant to D-T operation. The plasma edge densities can range from $\approx 0$ to $2.0 \times 10^{19} \frac{\text { part }}{\mathrm{m}^{3}}$ which can cover the 

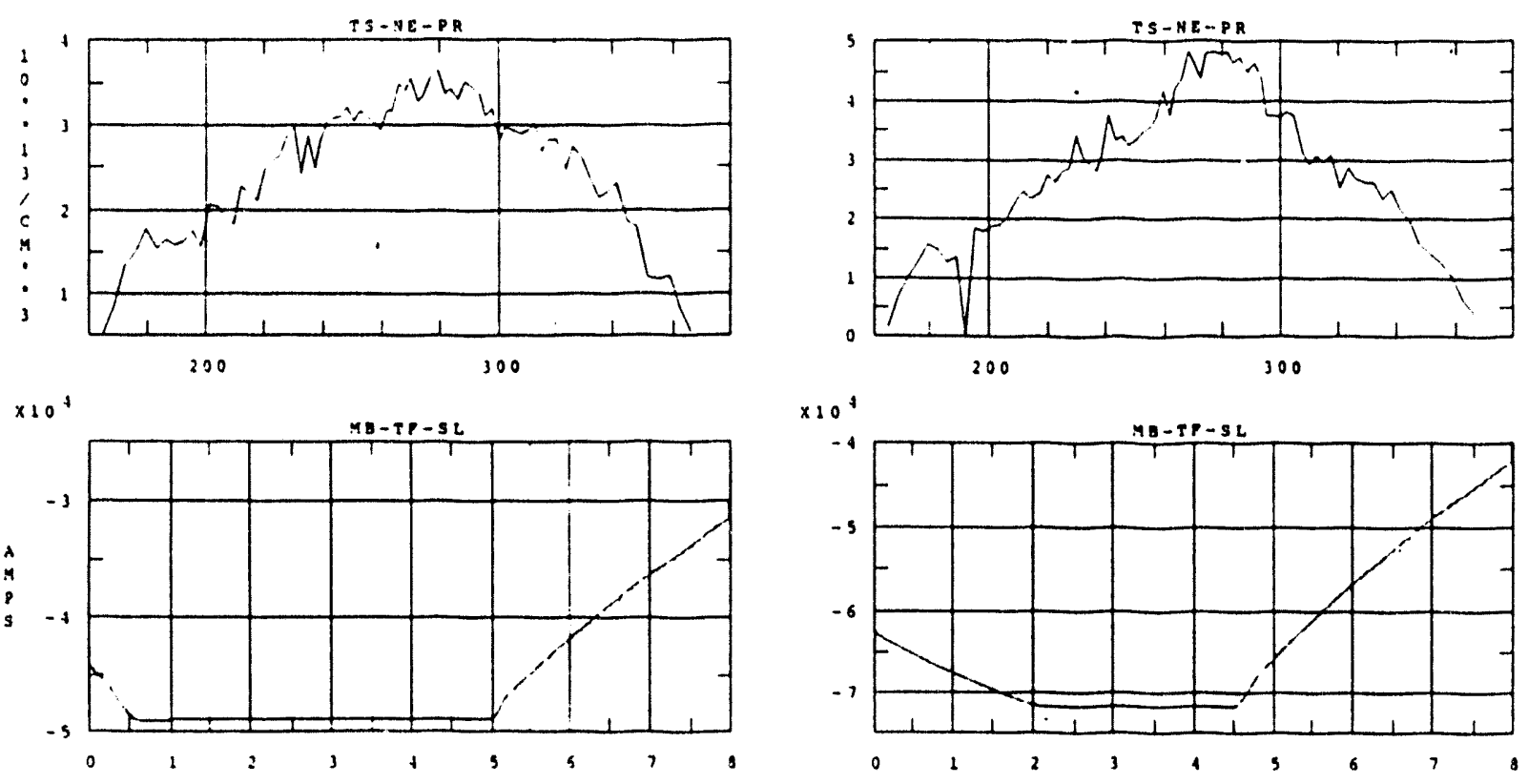

Figure 4.2: TFTR plasmas showing the density and toroidal current (magnetic field) range for two different plasma shots.

first 20 to $30 \mathrm{~cm}$ of the plasma edge depending on the magnetic configuration and the peak density of the plasma. The central magnetic field strength can range from $48 \mathrm{KAmps}$ (3.2 Teslas) to $i 2 \mathrm{KAmps}$ (4.5 Teslas). Example of TFTR plasma shots of these types of plasmas are shown in Figure 4.2 for low and high density plasmas and magnetic fields. This parameter range gave a frequency range of 90 to $120 \mathrm{GHz}$ which will probe the first 20 to $30 \mathrm{~cm}$ of the plasma edge (or even the plasma center if a low dencity and low magnetic field configuration is used). The selection of the $\mathrm{X}$-mode of propagation will reduce the required frequency range as explained previously. After studying different reflectometer designs and prospects, the final version of the electronic components is shown in Figure $4.1 .^{39,40,274,275}$

A two phase development program was sought at the time to prove the feasibility of the project. These are: 
1. Proof of principle phase: This allowed for a timely and low cost checkout of the dual frequency phase difference concept. By using the pre-existing waveguide of TFTR's reflectometer, shown in Figure $4.3^{277}$ and Figure A.1, a "test" of the reflectometer and technique could be done without going through the expense of building a waveguide run (Figure 4.4) to the ICRH antenna. This data provides a baseline of information about how the edge effects, if any, are modified well away from the ICRH antennas. This had some inconveniences, such as:

- The launcher for the TFTR fluctuation reflectometer was designed for probing highly localized fluctuations.

- Focused Gaussian beams are generated which intersect at the plasma midplane (see Figure 4.3).

- Remotely controlled mirrors are available to aim the beams, however it is difficult to make the beams cross at the edge, that is, the system is not optimized for plasma edge coupling, as can be seen from Figure A.2.

- The beam cross section may be too small to cover the entire profile distance of interest.

- Low reflected signal levels were frequently encountered.

- The launcher system is not close to the ICRH antennas, so that the effect of the ICRH power may not be seen directly.

2. Full system configuration: In this phase the reflectometer black box will be moved to a location in the basement under TFTR's vacuum vessel where it can reach the ICRH antenna mounted launcher, Figure $4.5,{ }^{276}$ which has been optimized for the operation of the reflectometer, Figure 4.6, and ICRH coupling studies. The radiation pattern of the reflectometer beam will not be the highly focused Gaussian beam from the TFTR fluctuation reflectometer, 
but will be a divergent beam pattern. This radiation pattern for the antenna is presented in Figure 4.6, where two perpendicular profiles of the pattern have been plotted. The coordinate system used for the plot is a spherical coordinate system, where the $z$-axis is in the direction of the propagation of the wave, the $x$-axis is along the long side of the waveguide, and the $y$-axis is along the short side of the waveguide. Thus, the pattern with the continous line is the pattern for an $x$-axis cross section, while the dotted line is the pattern for a $y$-axis cross section of the main pattern. This correspond to a cross section profile for $\phi=0$ (along the $x$-axis) and for $\phi=90$ degrees (along the $y$-axis), respectively. Thus, different from the TFTR fluctuation reflectometer system, this will be able to receive a good signal from the edge of the plasma but not from the center of the plasma.

The reflectometer black box has two main output ports. These are the I and $\mathrm{Q}$ signals of the quadrature detection system, given by:

$$
\begin{aligned}
I & =A_{a} \sin (\psi) \\
Q & =A_{a} \cos (\psi)
\end{aligned}
$$

where $A_{\mathrm{a}}$ is the amplitude of the signal, and $\psi$ is the phase of the wave. The quadrature detection system is a heterodyne system, which gives good signal to noise ratio and large dynamic range. A LeCroy 6810 is used to digitize these signals together with a ramp voltage proportional to the frequency of the swept frequency source. Some of the digitizer settings and parameters are presented on Table 4.1. The data is moved automatically by the PPPL computer system from the LeCroy 6810 memory to the designated storage media, where it can be accessed for analysis and reconstruction of the density profile. The phase difference is calculated by fringe counting the I and $Q$ signals from the raw data. The program allows for the special creation and processing of calibration data files, which can later be used to calibrate the general raw data files. 


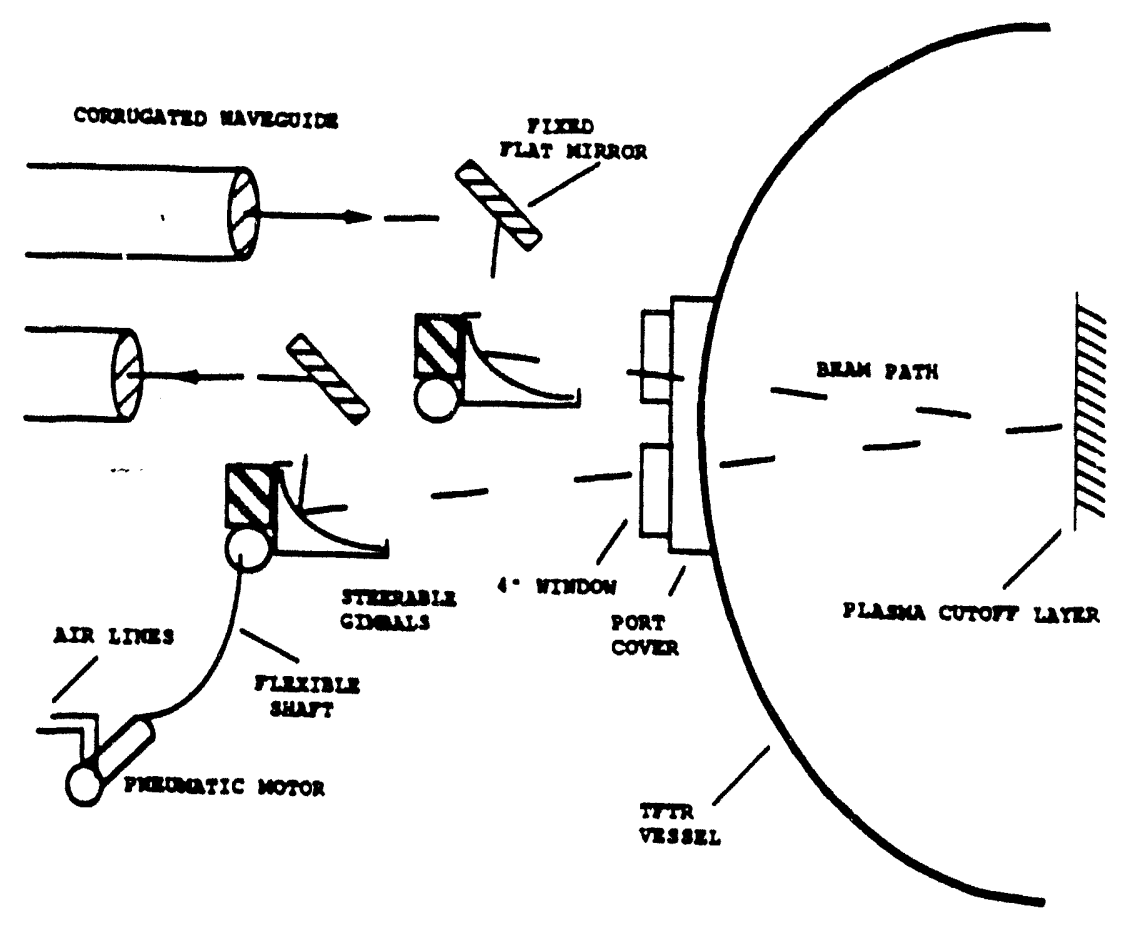

WAVEGUIDE TO VESSEL INTERFACE

Figure 4.3: TFTR fluctuation reflectometer waveguide to vessel interface.

\begin{tabular}{|l|r|}
\hline Description & LeCroy 6810 \\
\hline Number of channels & 4 \\
\hline Memory size per channel & $128 \mathrm{~K}$ \\
\hline Typical digitizing rate & $1 \mathrm{MHz}$ \\
\hline Data window & $128 \mathrm{msec}$ \\
\hline Sweep time & $1 \mathrm{msec}$ \\
\hline Repetition rate & $330 \mathrm{~Hz}$ \\
\hline Number of bits & 12 \\
\hline Voltage range & \pm 12.8 Volts \\
\hline Signal range & \pm 6.5 Volts \\
\hline
\end{tabular}

Table 4.1: Some of the digitizer major parameters. 


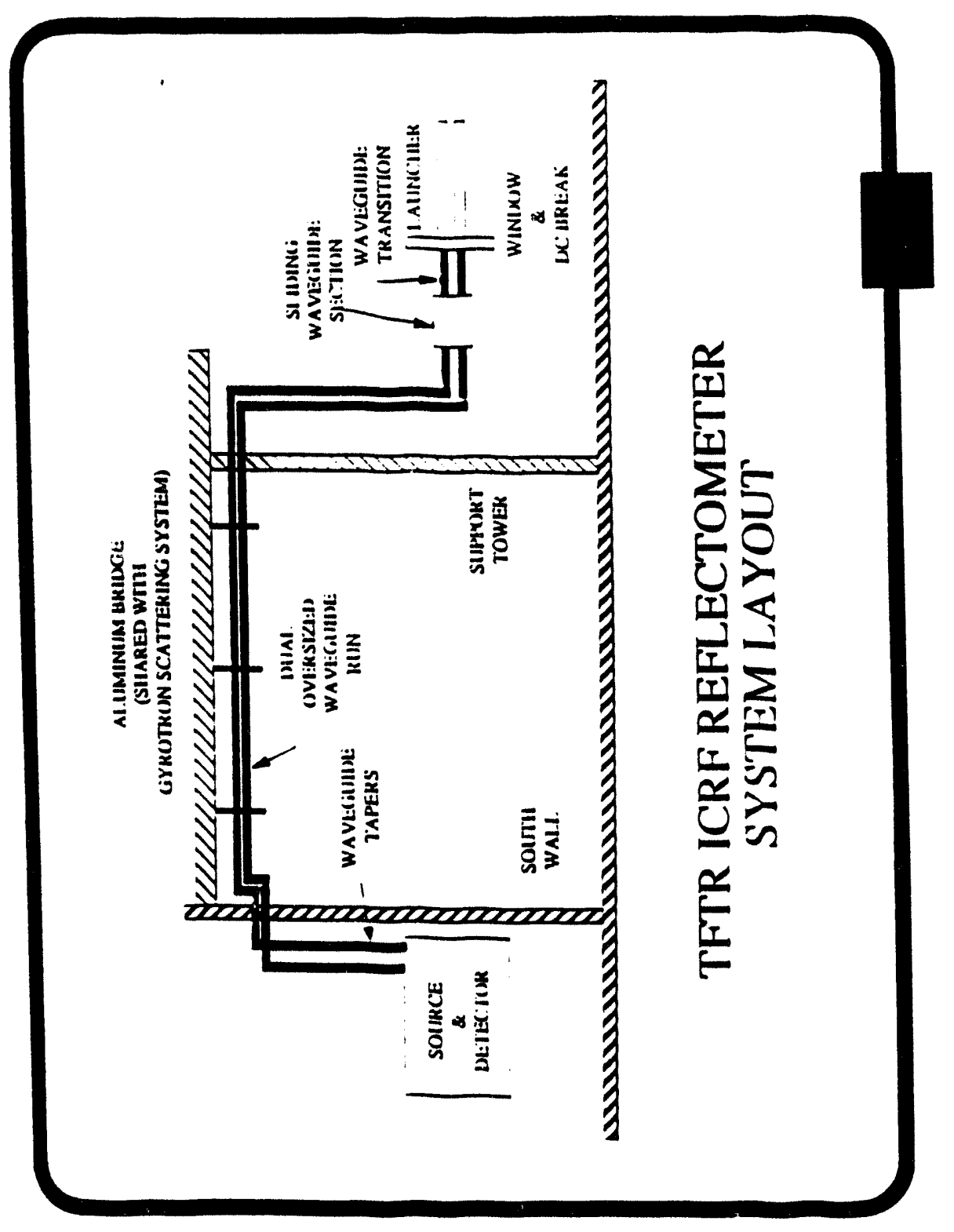

Figure 4.4: Layout of the reflectometer for the second phase of the project. 

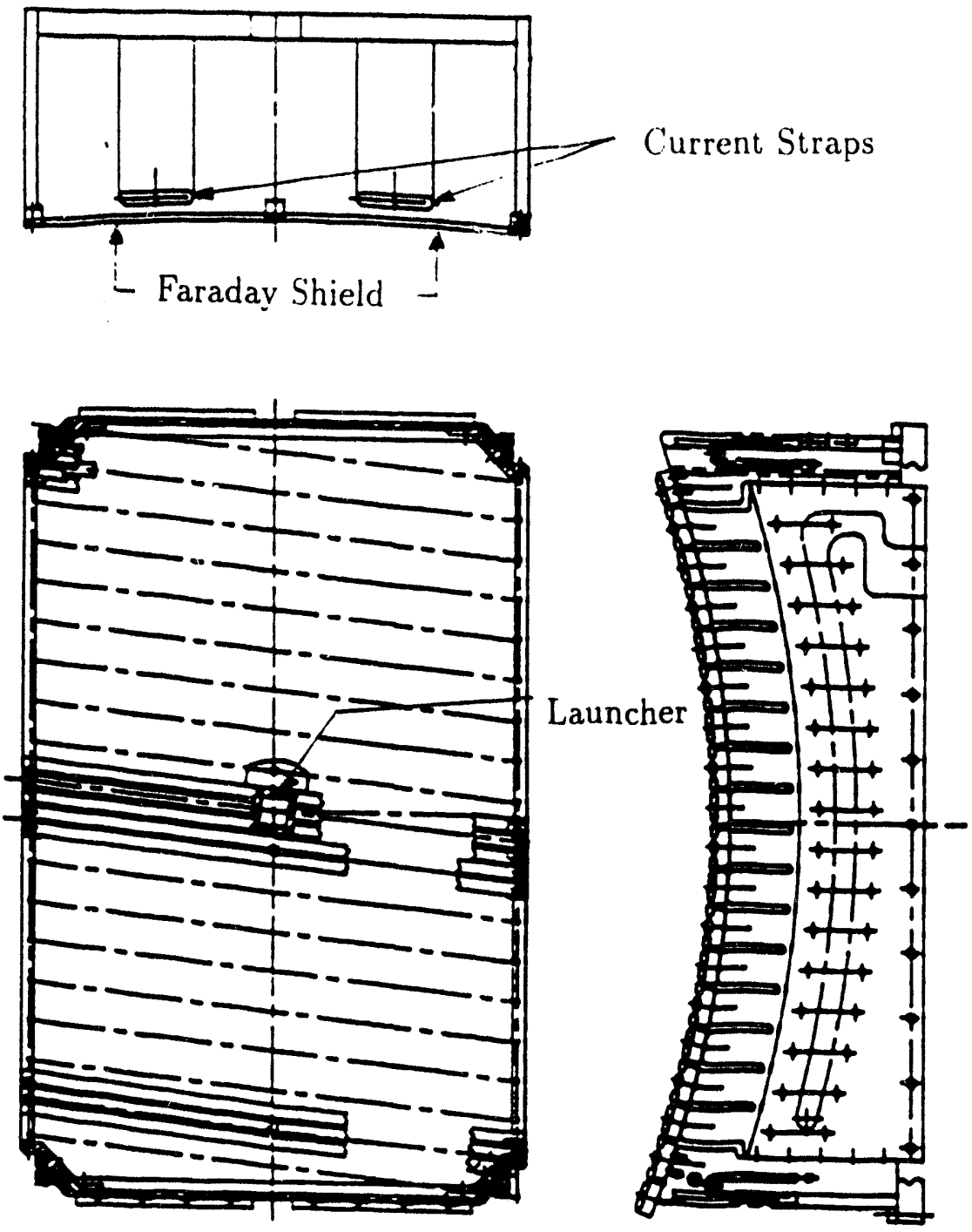

\section{ANTENNA RF BOX}

Figure 4.5: ICRH antenna RF box. This provides a front, side and top view of the antenna. The reflectometer launcher can be seen at the center of the antenna box. front view. The antenna current straps are shown at the top view of th drawing, thus the reflectometer launcher is between the current strá... 


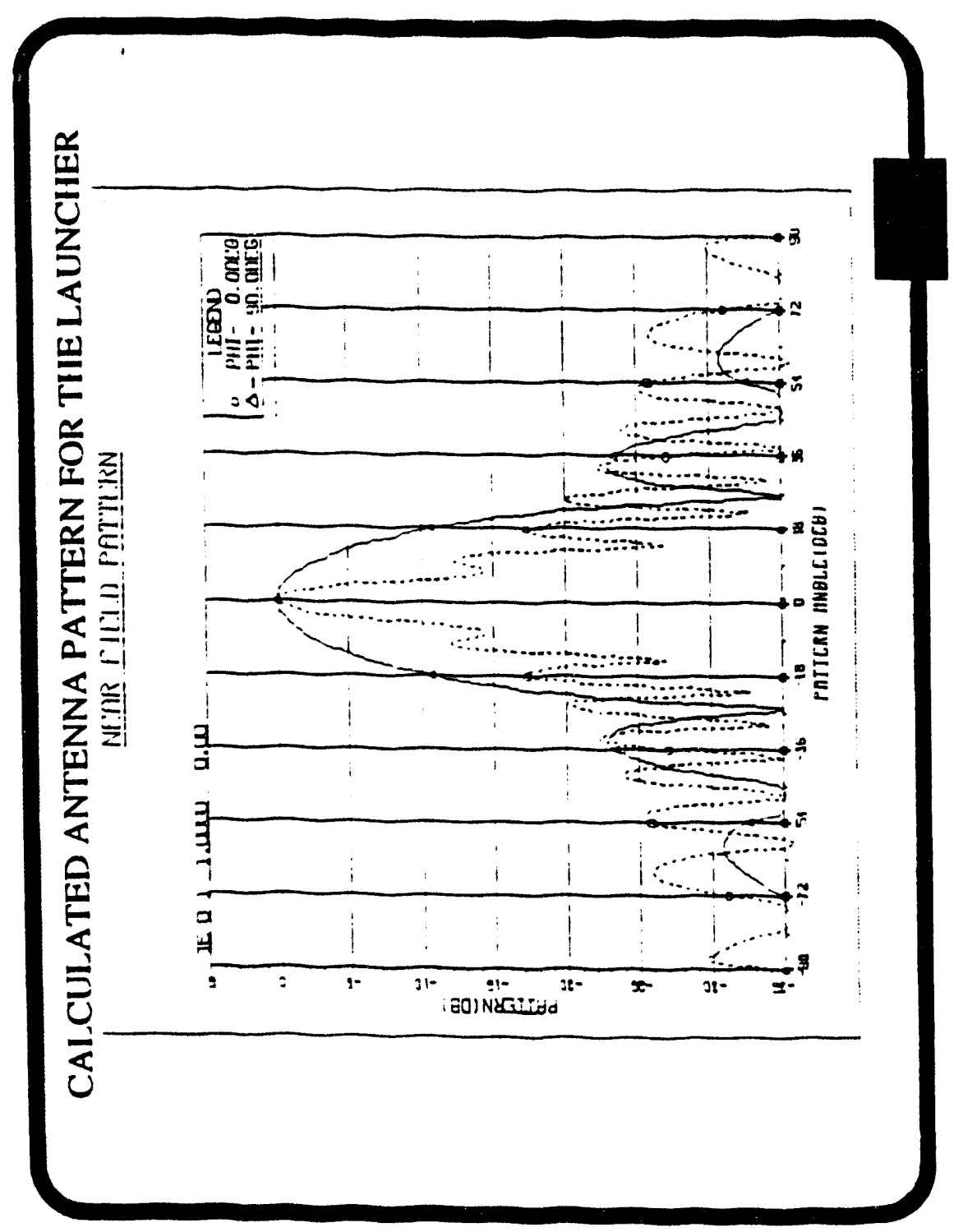

Figure 4.6: Radiation pattern of the launcher in two orthogonal directions, parallel $(\phi=0)$ and perpendicular $(\phi=90)$ to the widest dimension of the waveguide. 


\subsection{Description of the Reflectometer}

The reflectometer has one Backward Wave Oscillator (BWO) source in the frequency range of 8-12.4 GHz, w!lich provides the capavility for sweping the frequency of the probing beam. This signal is divided in three ways, as shown in Figure 4.1. The top two signals on the diagram go by almost equal circuit paths. The difference is the frequency of the up-converters $f 2$ and $f 3$. These frequencies are set to have a frequency difference equal to the desired $\Delta \omega$. After the signal goes through the up converters, it goes through the frequency doubler and tripler to increase the frequency of the probing beam to the range of 90 to $120 \mathrm{GHz}$. Finally, the two signals are added together (plasma probing signal) in a directional coupler where a small part of the probing signal is sampled to produce a reference signal (at the beat frequency) for the phase detector. The third signal (Local Oscillator or LO) that comes out of the BWO oscillator source, goes through a similar circuit path as the other two signals, except that the frequency of the up converter is different and the active tripler has been substituted by a $3^{\text {rd }}$ harmonic mixer where the plasma probing signal and the carrier signal are mixed together. This has the effect of down converting the two probing signals from the plasma to a frequency range where they can be easily amplified. Limiting amplifiers are use to remove amplitude modulations due to density fluctuations in the plasma and to provide proper signal levels to the mixer inpus. Then the two probing signals are mixed together in a mixer to obtain the phase difference between the two probing signals and fed into a quadrature phase detector system. The quadrature detection system provides phase information in the domain of 0 to $2 \pi$. The $I$ and $Q$ signals from the quadrature phase detection system are digitized together with the ramp voltage of the sweeper source. 


\subsection{Major Components of the Reflectometer System}

\subsubsection{Sources}

The main source of the reflectometer is a Backward Wave Oscillator (BWO) source. This can produce a wave in the frequency range of 8 to $12.5 \mathrm{GHz}$ with a sweeping time of $10 \mathrm{msec}$ with the build-in sweeper. An external sweeper source was used to reduce the sweeping time to $850 \mu \mathrm{sec}$ to reduce the effect of density fluctuations on the phase data.

The BWO is characterize for having a traveling wave RF circuit for which the energy of the traveling wave moves in the opposite direction of the phase velocity of the wave. This can be seen from Figure 4.7, where the frequency, $\omega$, as a function of the wave number, $k$, is plotted for a forward wave and backward wave. The phase velocity of a wave is defined by:

$$
V_{p}=\frac{\omega}{k}
$$

where $\omega$ is the frequency and $k$ is the wave number. While the group velocity is defined by:

$$
V_{g}=\frac{d \omega}{d k}
$$

which can be interpreted as the local value of the slope at a given point in the $\omega(k)$ curve. The phase information of the wave travels in the direction of the phase velocity, while the energy information of the wave travels in the direction of the group velocity. The curve in Figure $4.7 \mathrm{a}$ will give a postive value for the phase velocity, $V_{p}$, and a positive value for the group velocity, $V_{g}$, thus the phase information and the energy information of the wave travel in the same direction. On the other hand, for the plot in Figure $4.7 \mathrm{~b}$, the phase velocity has a positive value while the group velocity has a negative value because the slope of the curve at point " $A$ " is negative, thus the phase information of the wave moves in the positive direction while the energy information of the wave is moving in the negative direction. 
Figure 4.8 shows a simplified schematic of a Backward Wave Amplifier (BWA) which can be transformed to a BWO by means of a properly adjusted feedback network between the input and output terminals, as in any amplifier device. An electron beam is formed from the accelerated cathode emission by means of the potential $V_{a}$. The beam moves parallel to a uniform magnetic field $B_{0}$ that extends the length of the tube; the beam is then collected by the collector electrode. The collector potential, $V_{c}$, determines the beam current, $I_{b}$, and the potential $V_{a}$ determines the anode current, $I_{a}$. This arrangement permits separate control of the beam and helix (collector) currents. While the input signal travels toward the cathode, the electrons are bunched somewhere near the collector electrode. The bunched electrons interact with the helix slow wave in such a way as to cause the net energy to be transferred from the bunched electrons to the helix fieids. This permits wave amplification to occur. The input wave is moving in the negative direction (to the left of the figure), while the electron beam is moving to the positive direction (to the right). Since this is backward wave circuit, the phase velocity of the electron beam wave is in the positive direction, while the group velocity of the electron beam is in the negative direction, thus the energy of the electron beam (which is moving in the direction of the group velocity) is coupled to the input wave, causing amplification of the input wave. More information can be found in the references. ${ }^{280-282}$

\subsubsection{Directional couplers}

Directional couplers are four-port devices with the characteristic that power incident into port 1 will couple to port 2 (the through port) and to port 3 (the coupled port) but not to port 4 (the isolated port). The coupler is symmetric with respect to port 2 , that is, power incident into port 2 will couple to port 1 and port 4 but not to port 3 . Thus, port 1 and port 4 are decoupled, and port 2 and port 3 are decoupled. They are used to take samples of the signal on the input port without 


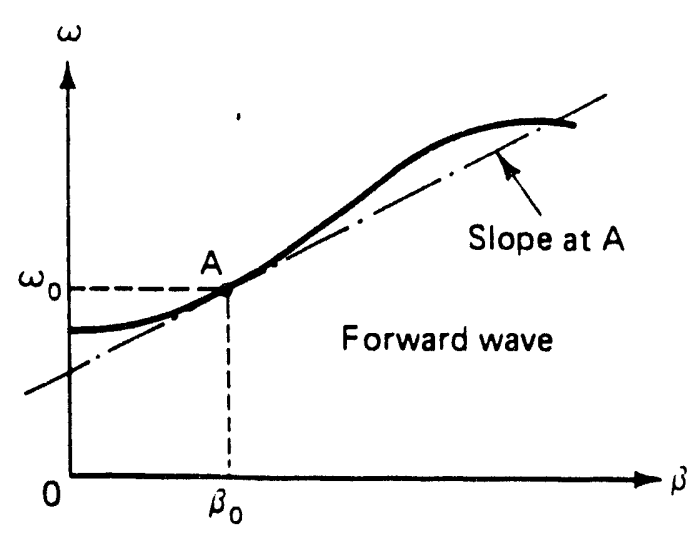

(a)

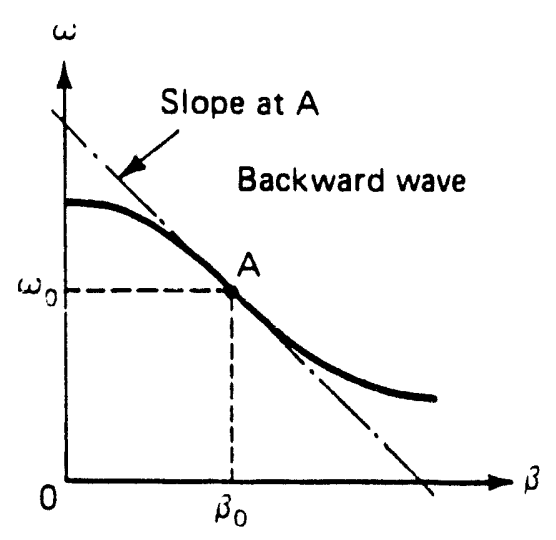

(b)

Figure 4.7: Plots of the dispersion relations $(\omega=\omega(k))$ for a forward wave (a) and a backward wave(b).

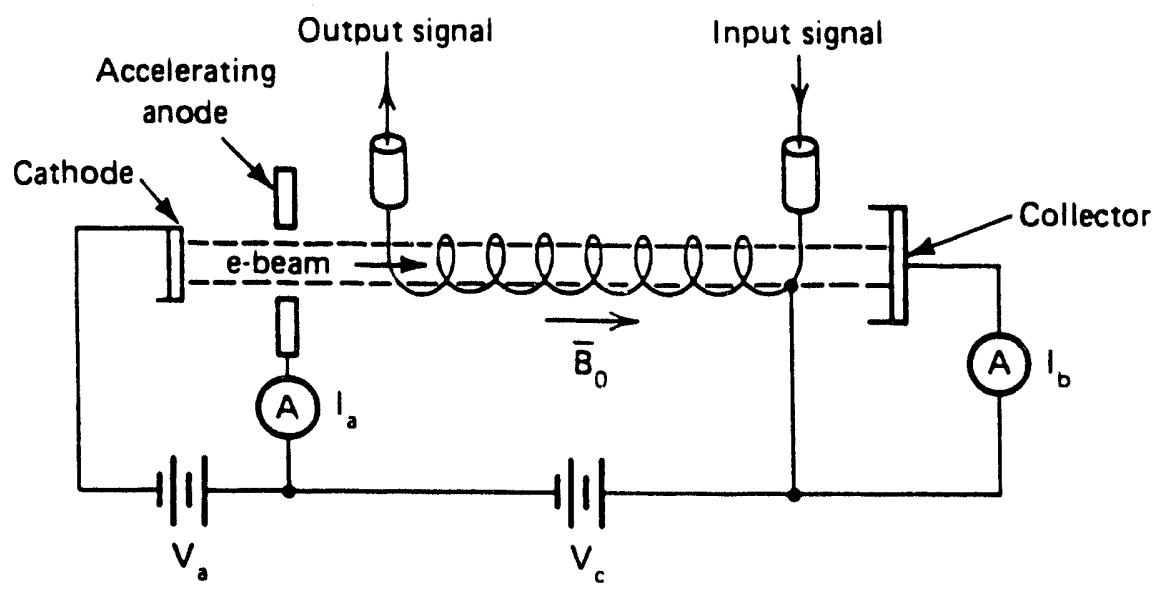

Figure 4.8: Backward wave amplifier schematic. 
disturbing or decreasing the signal going to the output port. The fraction of power from port 1 to port 3 is called the coupling factor, $C$, as defined by:

$$
C=10 \log \frac{P_{1}}{P_{3}}
$$

while the leakage of power from port 1 to port 4 is called the isolation, $I$, given by:

$$
I=10 \log \frac{P_{3}}{P_{4}}
$$

and the power fraction between port 3 and port 4 is called the directivity, $D$, given by:

$$
D=I-C=10 \log \frac{P_{3}}{P_{4}} .
$$

The ideal coupler is characterized solely by the coupling factor, since the isolation and directivity are both infinite. (In reality, directivities of 30-40 $\mathrm{db}$ are typical in the millimeter frequency range.)

\subsection{Summary}

This chapter presents the development, requirements, and description for the Dual Frequency Phase Difference reflectometer for TFTR. The unique environment of TFTR and the capability and final goal of using Tritium $\left({ }^{3} \mathrm{~T}\right)$ creates a series of constraints on the design parameters for a reflectometer. These were presented. The development of the reflectometer was divided into a two phase program. The first was a cheap and fast way to test the capabilities of the reflectometer by using the pre-existent waveguide system of the TFTR fluctuation reflectometer. This was used on a shared basis with the fluctuation reflectometer group (Dr. Ernesto Mazzucato and Dr. Rafi Nazikian). From this waveguide system it was possible to collect data on the plasma at a position away from the ICRH antenna; thus, no direct measurements of the density changes at the ICRH antenna environment could 
be made, but it could measure any global changes in the edge density profile and in the center of the plasma (for different values of the magnetic field, $B_{0}$ ) thanks to the highly focused Gaussian beams of the TFTR fluctuation reflectometer system. This provided a base line data base for the performance of the reflectometer. After a successful proof of principle test on the TFTR fluctuation reflectometer system, the Dual Frequency Phase Difference reflectometer will be ready for the second phase of the project. A description of the reflectometer black box components and how these components generate the dual frequency probing signal used for the measurement was discussed. 


\section{CHAPTER V}

\section{SUMMARY}

Reflectometry is a technique that relies on the total reflection of an electromagnetic microwave signal to obtain information about the density profile and the density fluctuations of the plasma. This reflection occurs when the local index of refraction of the wave goes to $0, n \rightarrow 0$, which is equivalent to the wavelength going to infinity, $\lambda \rightarrow \infty$, or the phase velocity going to infinity, $v_{\text {phase }} \rightarrow \infty$. This defines the cutoff of a wave. Depending on the orientation of the wave's electric field with respect to the plasmas magnetic field, the wave will be classified as the Ordinary wave $(E \| B)$, with a cutoff given by:

$$
\omega_{O}=\left(\frac{n_{e} q_{e}^{2}}{\epsilon_{0} m_{e}}\right)^{\frac{1}{2}}
$$

or as an eXtraordinary wave $(E \perp B)$ with a cutoff given by:

$$
\omega_{X}= \pm \frac{\omega_{e c}}{2}+\left(\omega_{p e}^{2}+\left(\frac{\omega_{e c}}{2}\right)^{2}\right)^{\frac{1}{2}}
$$

where $\omega_{\mathrm{ec}}$ is the electron cyclotron frequency of the plasma and $\omega_{p e}$ is the plasma electron frequency. Either one of these two modes of propagation can be used to probe the plasma and obtain the density data.

Reflectometry has many advantages over other density profile diagnostics, such as Thomson Scattering, Multichannel Infra Red Interferometer, Langmuir probes and Heavy Ion Beam Probe. It requires little viewing access to the plasma, it is non-disturbing to the plasma (i.e., it requires no structures in the plasma as compared to Langmuir probes), can probe the edge as well as the center of the plasma, 
its components are relative cheap and available, it provides a remote measurement as opposed to a intrusive measurement (like Langmuir probes), can provide continuous time density profiles (as opposed to a one shot profile like Thomson Scattering) and can provide density fluctuation information as well as density profile information depending on the technique being used. These characteristics makes it ideal for measuring the changes of the edge density profile in from of the ICRH antenna due to the power deposition on the plasma. In general Langmuir probes can not survive the high temperatures and densities of the plasma in front of the ICRH antenna. Viewing access for diagnostics like Thomson Scattering and MIRI is generally insufficient in front of the ICRH antenna. As it has been previously discussed, the edge density profile in front of the ICRH antenna can be modified by Ponderomotive forces, impurity production, sheath rectification effects, self sputtering, quiver induced losses, etc... This has the effect of changing the loading and therefore the coupling of the ICRH power to the plasma. A reflectometer is the first choice to do a direct measurement of the changes on the plasma edge density profile in front of the ICRH antenna due to these phenomena. The information obtained from the reflectometer can be used to better understand the plasma edge and to maximize the coupling and power deposition to the plasma, which is of great importance to the success of ICRH as a primary source of auxiliary heating and to the achievement of temperatures at the fusion reactor regime.

This report presented a library search in the field of reflectometry, with emphasis on the techniques to measure density profiles. The first chapter provides an introduction to fusion research and fusion experiments, along with a description of the Tokamak Fusion Test Reactor (TFTR), the machine where the new reflectometer will be installed and where data will be collected. Chapter two starts with a short history of the origins of reflectometry and continues with a detailed derivation of the formulae required to understand the basics of electromagnetic wave propagation 
propagation in plasmas and reflectometry theory. The different techniques and approaches used by reflectometry to obtain density information (fluctuations and/or density profiles) are discussed, and a description of the new Dual Frequency Phase Difference technique is presented. A description of the possible complications and error sources is given. Because the main purpose of this reflectometer is to measure and study edge density changes in front of an ICRH antenna due to the ICRH power deposition, chapter three covers the general theory of ICRH and the different phenomena that can induce changes on the plasma edge density. The description of different ICRH antennas used in fusion research is presented, with detailed drawings of these antennas and their respective experiments. A description and parameters for one of the TFTR antennas is presented, together with a list of references and a table of parameters for the antenna. Chapter four presents a description of the Dual Frequency Phase Difference reflectometer designed by ORNL for TFTR. The development and description of how it works is discussed. In the appendices a discussion of the theory of Gaussian beam optics for lasers and of Gaussian beams in a plasma is presented. This theory is of importance to this work because during the first phase of the project, the reflectometer will be using the TFTR fluctuation reflectometer waveguide system. This system uses a set of parabolic mirrors to direct and focus the highly localized Gaussian beam of the waveguide. Thus, this theory is used to calculate the intersection area or cross section of the beams such that an idea of the signal amplitude for the reflectometer can be estimated.

The Dual Frequency Phase Difference reflectometer has excellent potentials for the study of the plasma edge and in particular to study the induced changes of the ICRH antenna and power deposition on the plasma edge as a function of time on a shot by shot basis. The information about the edge density profile in front of the ICRH antenna can be used to maximize the power coupling and effectiveness of the ICRH antenna. The used of the X-mode of propagation (instead of the O-mode) has 
the advantage of been able to probe the plasma edge with a minimum of frequency range starting with the local electron cyclotron frequency of the plasma at the edge. 


\section{APPENDIX A \\ Gaussian Beam Optics}

\section{A.1 Pre-History of Lasers}

During the first testing phase, the dual frequency reflectometer used TFTR's reflectometer setup. ${ }^{38}$ This consists of the waveguide, reflecting mirrors, and focusing mirrors, as can be seen from Figure A.1. This system uses focused Gaussian beams, as shown in Figure A.2 and in Figure A.3, which intersect at the plasma midplane. It was designed for probing highly localized fluctuations at the center of the plasma. Because of the optical nature of this setup, an understanding of general optics and Gaussian optics (which governs the propagation of the beam) is necessary. The development of the theory and corresponding formulae has been done in the field of laser beams and resonators. Therefore, a discussion of laser optics and Gaussian beams will be presented from the point of view of laser beams and resonators, but with direct application to the propagation of the reflectometer beam through TFTR's setup. Before continuing, it should be made clear that the words laser beam and/or reflectometer beam and/or microwave beam will be used as synonymous throughout this appendix.

The idea of the laser resonator originated when R. Dicke, ${ }^{257}$ A. Prokhorov, ${ }^{258}$ and A. L. Schawlow and C. H. Townes, ${ }^{259}$ independently proposed the use of the Fabry-Perot interferometer as a laser resonator. The modes of propagation of such a structure as determined by diffraction effects was first calculated by A. G. Fox and T. Li. ${ }^{260,261}$ G. D. Boyd and J. P. Gordon, ${ }^{262}$ and G. D. Boyd and H. Kogelnik, ${ }^{263}$ 


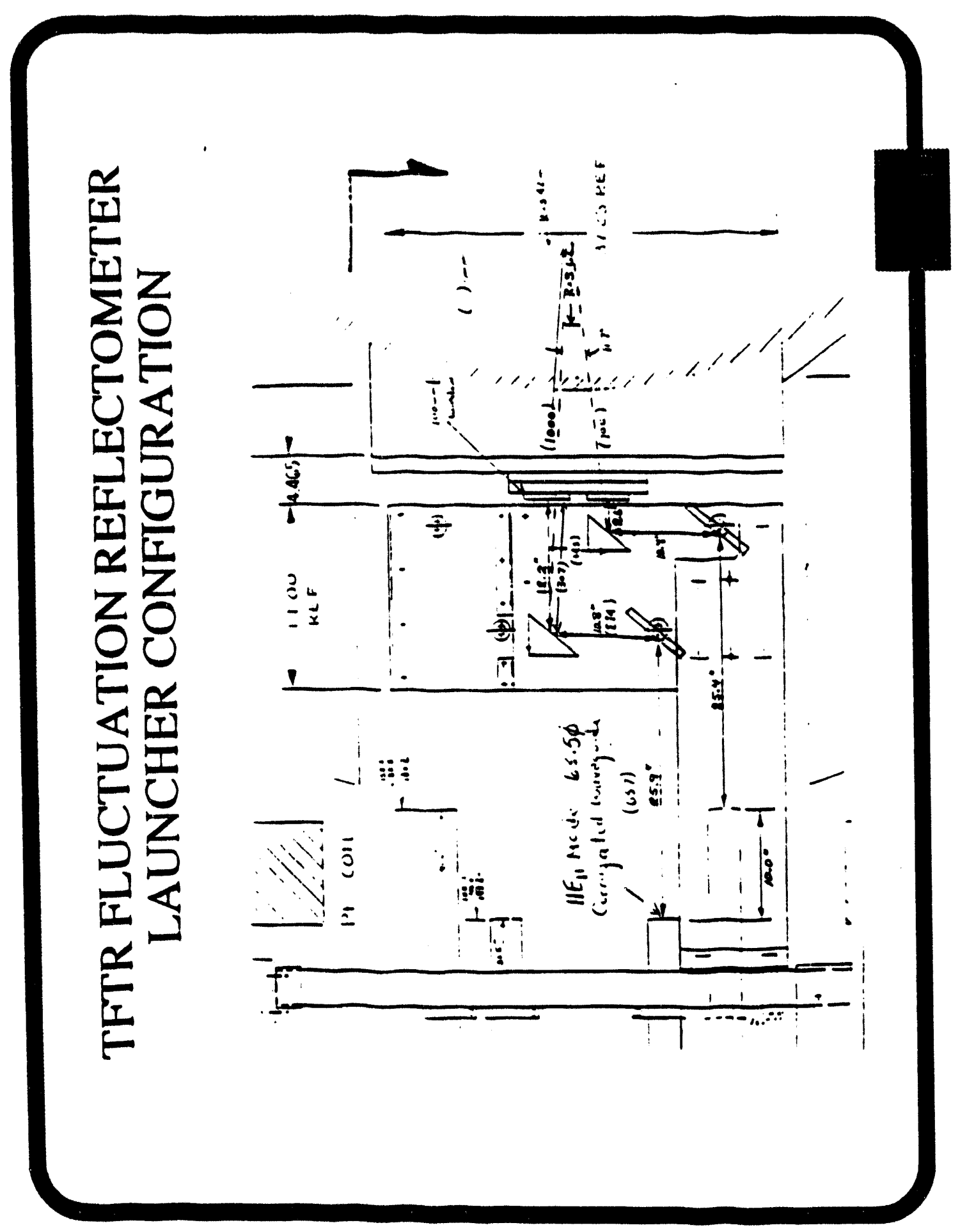

Figure A.1: TFTR's mirrors setup and geometry. 


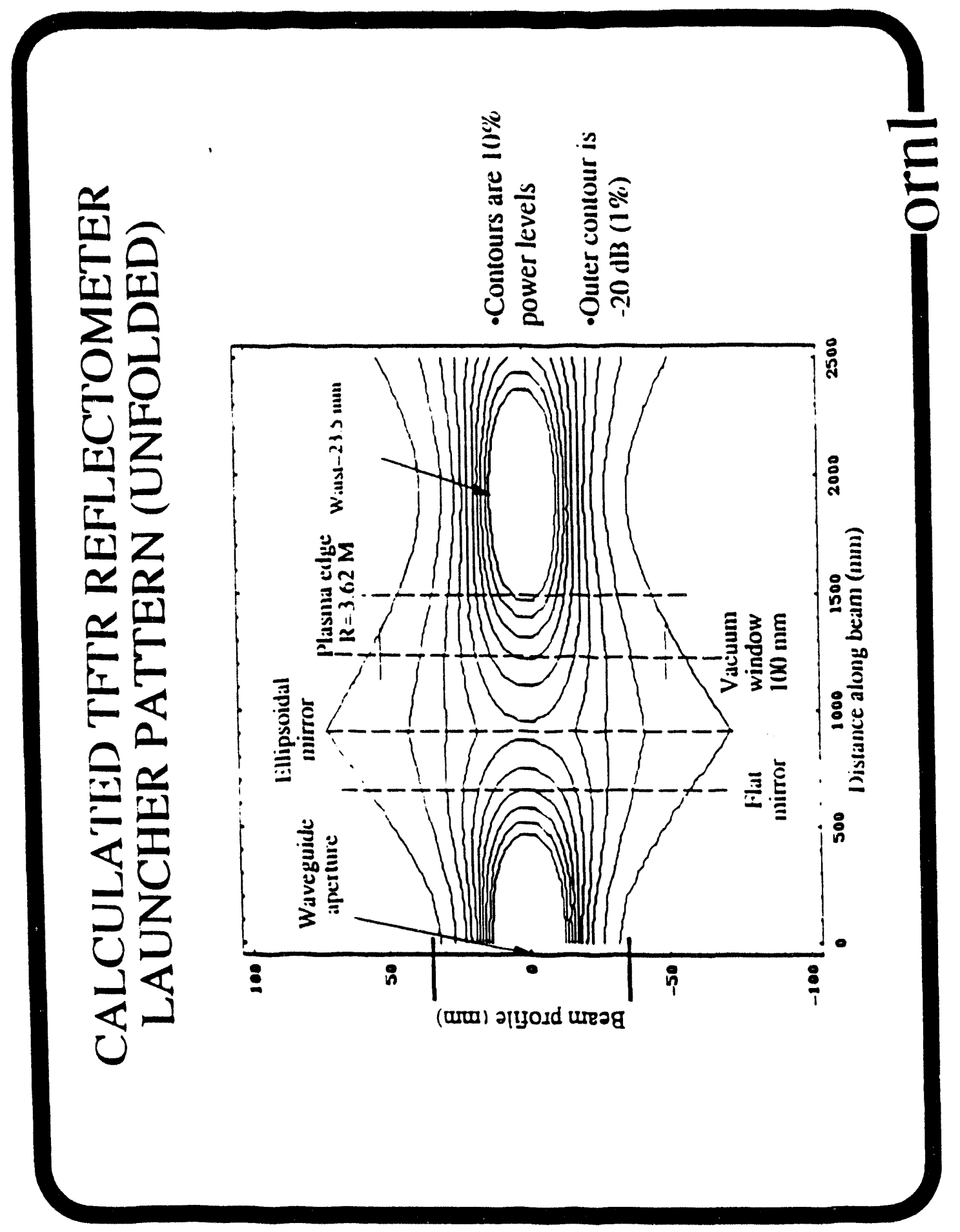

Figure A.2: Calculated TFTR's reflectometer launcher Gaussian pattern (unfolded). 


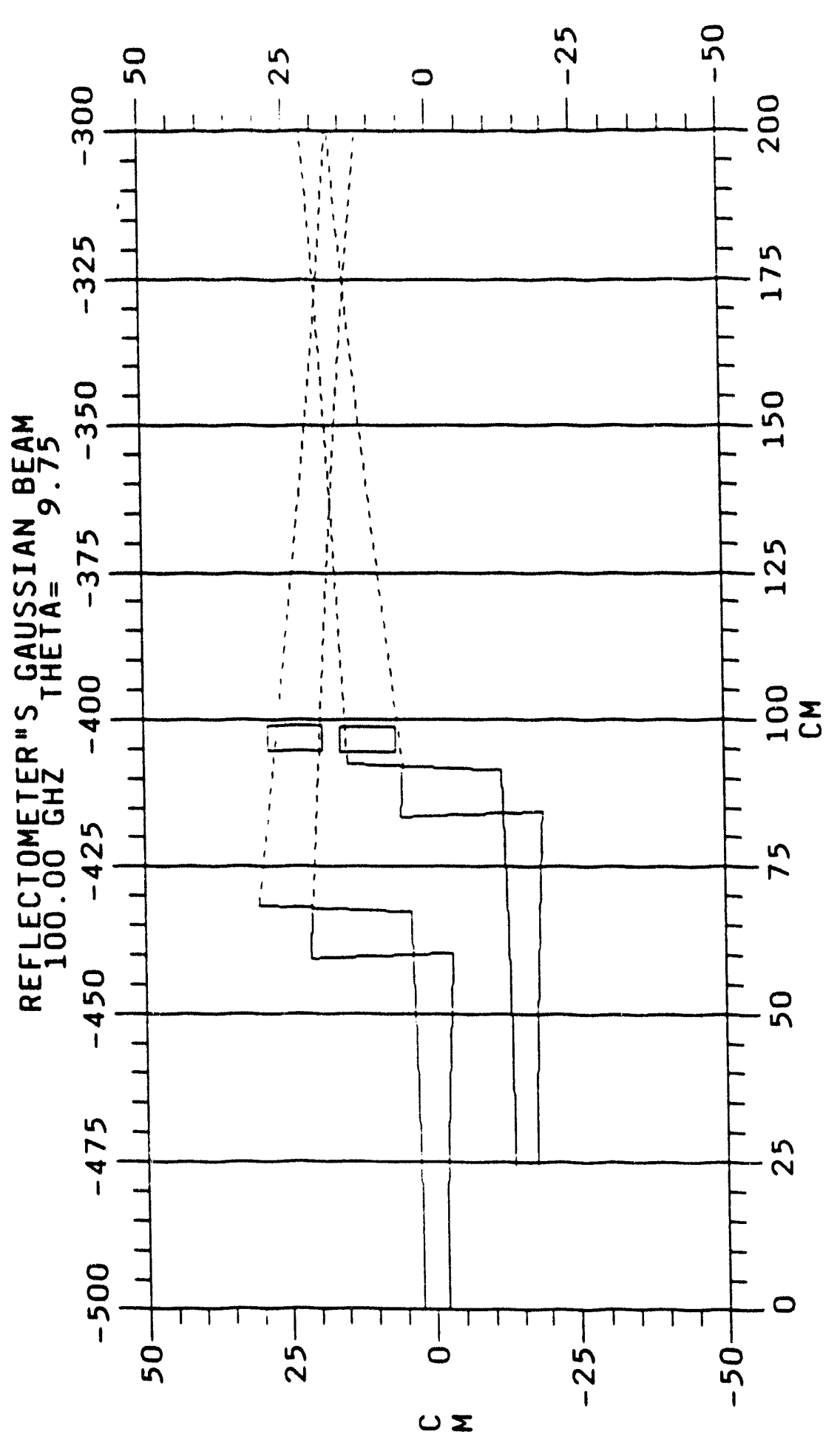

Figure A.3: Calculated TFTR's beam geometry and intersection inside the vacuum chamber. 
developed the theory for a resonator with spherical mirrors and approximated the modes by wave beams. The concept of electromagnetic wave beams was also introduced by G. Goubau and F. Schwering, ${ }^{264}$ who investigated the properties of sequences of lenses for the guided transmission of electromagnetic waves. Another treatment of wave beams was given by J. R. Pierce. ${ }^{265,266}$ The behavior of Gaussian beams as they interact with various optical structures has been analyzed by G. Goubau, ${ }^{267}$ H. Kogelnik ${ }^{268}$ and others. ${ }^{269}$

\section{A.2 Paraxial Ray Analysis}

The study of the propagation of paraxial rays through an optical system with resonators, transmission lines (TFTR's setup in our case), and other similar structures can reveal many important geometrical properties of the system, such as the optical stability of the structure (resonators) ${ }^{263}$ and the loss of unstable resonators. ${ }^{270}$ The propagation of a paraxial ray and/or of a Gaussian beam through one or more optical structures can be described by the use of ray transfer matrices. The development of H. Kogelnik and $\mathrm{T}$. $\mathrm{Li}^{278}$ will be followed here. A more detailed treatment of ray propagation can be found in Born and Wolf, ${ }^{279} \mathrm{~W}$. Brower, ${ }^{271}$ and others. ${ }^{272,273}$

\section{A.2.1 Ray transfer matrix}

A paraxial ray in an optical system is characterized by its distance, $x$, with respect to the optic $z$-axis of the system and its angle or slope, $x^{\prime}$, with respect to the axis of the system, as is shown in Figure A.4. ${ }^{268}$ The slope, $x^{\prime}$, is assumed to be small. The optical path of the ray depends on the characteristics of the optical system as well as the initial position and slope of the ray, i.e., $\left(x, x^{\prime}\right)$. Assuming that the optical system has linear response, then the output ray in terms of the input 
ray can be expressed as a matrix times a vector type of equation, that is:

$$
\left(\begin{array}{l}
x_{2} \\
x_{2}^{\prime}
\end{array}\right)=\left(\begin{array}{ll}
A & B \\
C & D
\end{array}\right) \cdot\left(\begin{array}{l}
x_{1} \\
x_{1}^{\prime}
\end{array}\right)
$$

where the $A B C D$ matrix is called the ray transfer matrix and is defined such that its determinant is equal to unity:

$$
\left|\begin{array}{ll}
A & B \\
C & D
\end{array}\right|=A D-C B=1
$$

This can be used to express the ray transfer matrix in terms of any 3 given parameters.

The elements of the ray transfer matrix can be related to the focal length, $f$, and the location of the principal planes of the system by the formulas:

$$
\begin{aligned}
f & =\frac{-1}{C} \\
h_{1} & =\frac{D-1}{C} \\
h_{2} & =\frac{A-1}{C}
\end{aligned}
$$

where $h_{1}$ and $h_{2}$ are the distances of the principal planes from the input and output planes as shown in Figure A.4. The transfer matrixes of six elementary optical structures are presented in Table A.1. ${ }^{278}$ They represent the following:

1. The ray transfer matrix of a ray over a distance, $d$, with input and output planes at positions 1 and 2 .

2. The ray transfer matrix of a ray going through a thin lens of focal length $f$.

3. The combination of 1 and 2. The matrix is obtained by multiplying matrix 1 by matrix 2 .

4. A beam passing through two structures of type 3 . 


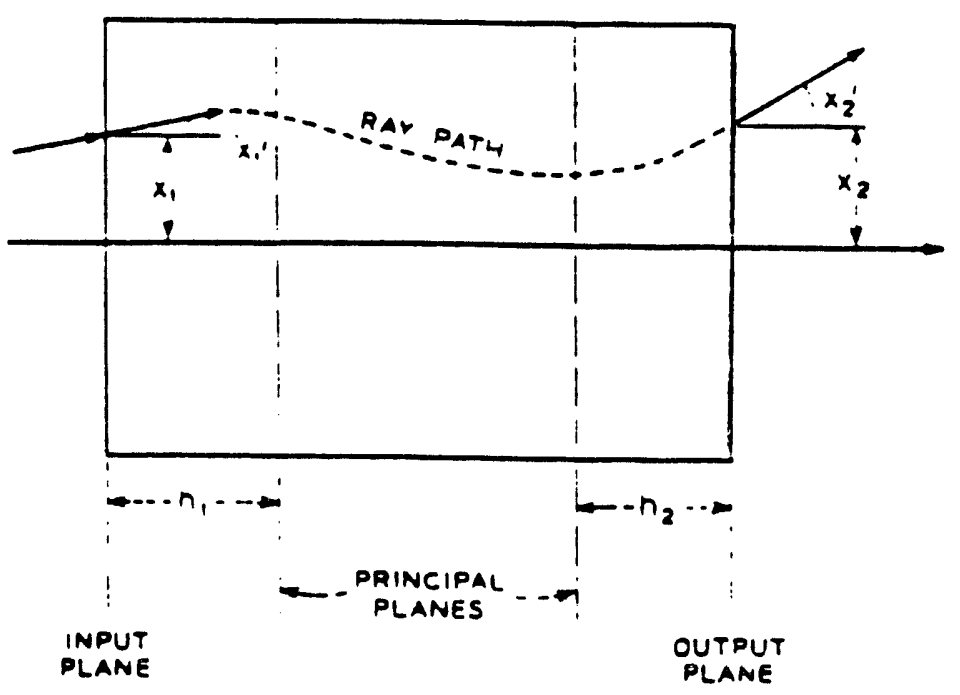

Figure A.4: Optical system diagram and parameters. An arbitrary ray and its corresponding parameters are also shown. (C)1966 IEEE 
Rai Transfia Matrices of Six Elementary Omte.ll Strcctiris

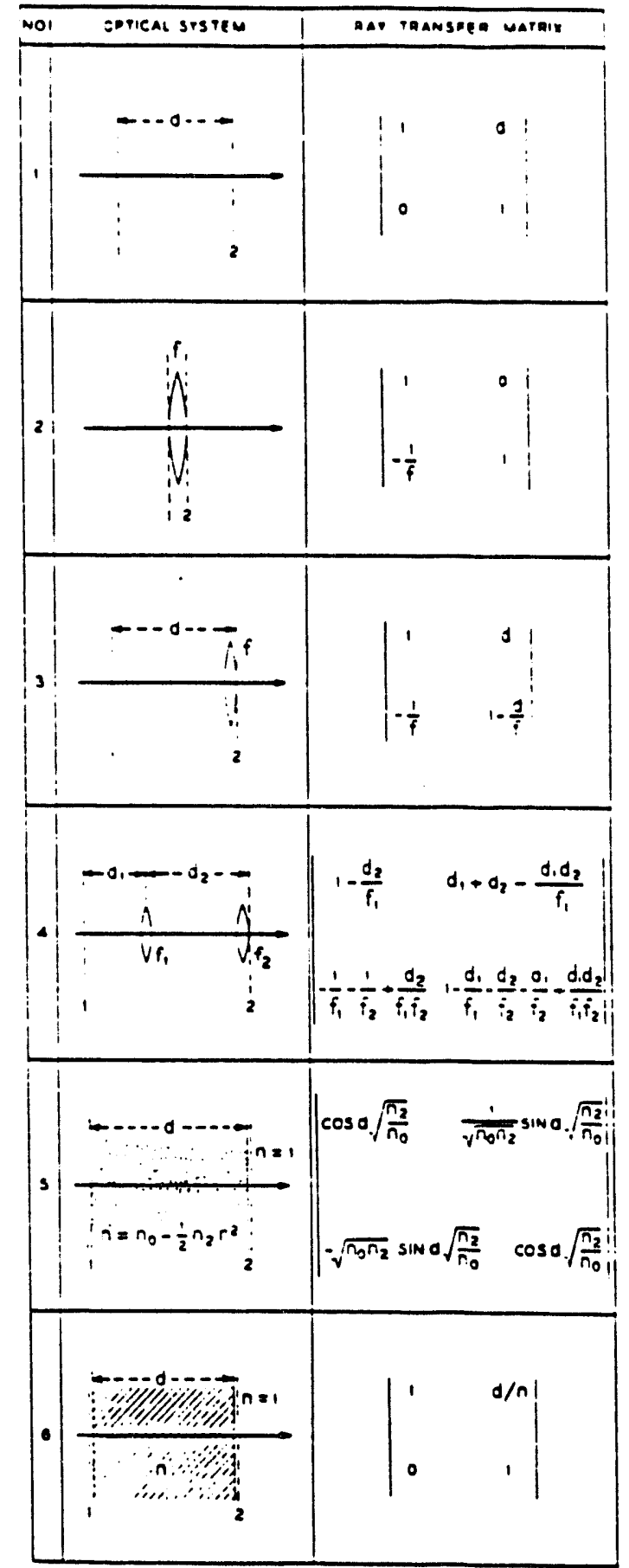

Table A.1: Ray transfer matrices of six elementary structures. (c) 1966 IEEE 
5. The ray transfer matrix of a lens like medium (like a laser). It is characterized by a parabolic radial density profile.

6. The matrix for a dielectric material of index of refraction $n$ and length $d$.

\section{A.2.2 Periodic sequences}

A laser resonator cavity can be modeled by means of two spherical mirrors facing each other. Such a system will have a focusing effect on the light rays as they bounce back and forth between the two mirrors. The two spherical mirrors facing at each other are equivalent to an infinite sequence of equal lenses, in which the $n^{\text {th }}$ bounce of the ray on one of the spherical mirrors is equivalent to the same ray passing through the $n^{\text {th }}$ lens of the infinite sequence of lenses. ${ }^{268}$ A schematic of the infinite sequence system is shown in Figure A.5 for an arbitrary ABCD matrix. The ray transfer through the $n^{\text {th }}$ element (or reflection) is described by the $n^{\text {th }}$ power of this matrix. This can be evaluated by means of Sylvester's theorem, ${ }^{268}$ which is:

$$
\left|\begin{array}{ll}
A & B \\
C & D
\end{array}\right|^{n}=\frac{1}{\sin \Theta}\left|\begin{array}{cc}
A \sin (n \Theta)-\sin ((n-1) \Theta) & B \sin (n \Theta) \\
C \sin (n \Theta) & D \sin (n \Theta)-\sin ((n-1) \Theta)
\end{array}\right|
$$

where the angle $\Theta$ is defined by:

$$
\cos \Theta=\frac{1}{2}(A+D)
$$

Based on equations A.6 and A.7 optical systems can be classified as either stable or unstable. Systems that have a real angle $\Theta$ are stable, while systems which do not have a real angle $\Theta$ are unstable. Mathematically speaking, any matrix which satisfies the inequality:

$$
-1<\frac{1}{2}(A+D)<1
$$

is stable, and any system not satisfying the above inequality is unstable. 


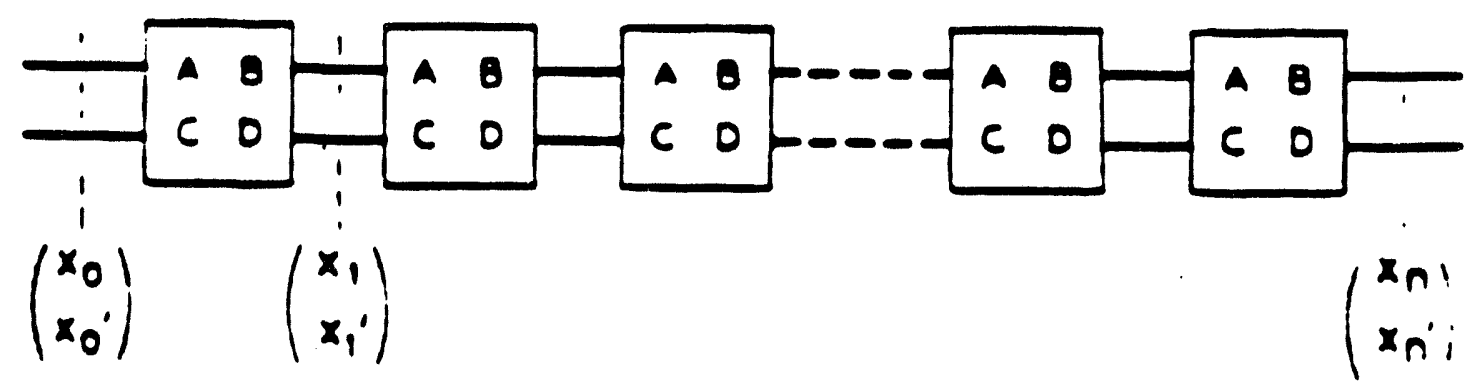

Figure A.5: Diagram of an infinite sequence of systems (lenses) each characterized by an equal ray transfer matrix. (c) 1966 IEEE

\section{A.3 Wave Analysis of Laser Beams}

This section will discusses laser beams from the point of view of satisfying a wave equation, thus taking into account the wave nature of the beam. The results derived here are applicable to optical systems with large apertures as compared to the diameter of the beam and/or the wavelength of the wave. This means that edge diffraction effects have not been taken into account. A discussion of the effects of edge diffraction can be found in the references. ${ }^{278}$ The study of "beam waves" was first made by Boyd and Gordon, ${ }^{262}$ and by Goubau and Schwering. ${ }^{264}$ Here the development of Kogelnik ${ }^{278}$ and others ${ }^{269}$ will be followed.

\section{A.3.1 Solution to the beam's wave equation}

Laser beams are similar to plane waves (since both have to satisfy the wave equation), except that their intensity distribution is not uniform and their phase fronts are slightly curved. Thus, a laser beam traveling in the $z$-direction can be 
expressed as:

$$
u=\psi(x, y, z) e^{-i k z}
$$

where $\psi(x, y, z)$ is a slow varying function of $z$ and represents the differences between the laser beam and a plane wave. It incorporates the idea of a non-uniform intensity distribution, the expansion of the beam as it moves away from the source, the curvature of the phase front, and any other difference. This laser beam function is bound to satisfy the wave equation given by:

$$
\nabla^{2} u+k^{2} u=0
$$

assuming harmonic solutions, where $k=\frac{2 \pi}{\lambda}$ is the propagation constant in the medium and $\lambda$ is its wave length. Substituting the representation of the beam wave, equation A.9, into the wave equation, equation A.10, gives:

$$
\frac{\partial^{2} \psi}{\partial x^{2}}+\frac{\partial^{2} \psi}{\partial y^{2}}-2 i k \frac{\partial \psi}{\partial z}=0
$$

where terms of order $\frac{\partial^{2} \psi}{\partial z^{2}}$ have been ignored because it was assumed that $\psi(x, y, z)$ was a slow changing function of $z$.

The differential "beam equation" has a form similar to the ubiquitous time dependent Schrödinger equation. So, borrowing one of the Schrödinger solutions

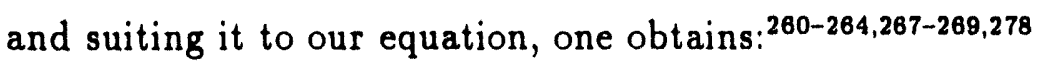

$$
\psi=\exp \left(-i\left(P(z)+\frac{k r^{2}}{2 q(z)}\right)\right)
$$

with

$$
r=\left(x^{2}+y^{2}\right)^{\frac{1}{2}}
$$

as a solution to the beam equation. The parameter $P(z)$ represents a complex phase shift which is associated with the propagation of the light beam and $q(z)$ is a complex beam parameter which describes the Gaussian dependence of the beam with respect to the distance from the beam's main axis (assumed to be in the $z$-axis). Equations 
for $P(z)$ and $q(z)$ can be obtained by substituting the beam function, equation A.12, into the beam equation, equation A.11, and comparing terms of equal powers in $r$. This will yield:

$$
q^{\prime}(z)=1
$$

and:

$$
P^{\prime}(z)=\frac{i}{q}
$$

where the prime symbol, ', indicates differentiation with respect to the argument, z. Integration of equation A.14 gives:

$$
q_{2}=q_{1}+z
$$

which relates the parameter $q_{2}$ of the output plane to the parameter $q_{1}$ of the input plane where the planes are separated by a distance $z$.

\section{A.3.2 Propagation law for the fundamental mode}

The beam function, equation A.12, is not the only known solution to the beam equation, but is probably the most important. This beam function is called the fundamental mode. Higher order modes are discussed in the references, such as: A. G. Fox, ${ }^{280,281}$ G. D. Boyd, ${ }^{262}$ H. Kogelnik, ${ }^{288,268,278}$ E. L. O'Neill ${ }^{272}$ and C. S. Williams. ${ }^{273}$ Because of the importance of the fundamental mode it will be discussed here in more detail.

Start by redefining the complex $q(z)$ parameter in terms of two real parameters, $R$ and $w$, as:

$$
\frac{1}{q(z)}=\frac{1}{R(z)}-\frac{i \lambda}{\pi w^{2}(z)}
$$

where $R(z)$ represents the radius of curvature of the wave front that intersects the $z$-axis at $z$, and $w(z)$ is a measure of the decrease of the intensity with respect to the distance from the axis (i.e., the $e$-folding distance of the Gaussian). The new parameter is often called the beam radius or spot size, and $2 w$ is the beam diameter. 
A focused Gaussian beam contracts to a minimum diameter of $2 w_{0}$ at the point that is called the beam waist, where the phase front is plane (so that $R \rightarrow \infty$ ). If $z$ is measured from this waist, the $q$ parameter becomes purely imaginary, that is:

$$
q_{0}=\frac{i \pi w_{0}^{2}}{\lambda}
$$

and at a distance $z$ from the waist $q$ is given by:

$$
q(z)=q_{0}+z
$$

or using equation $A .18$, as:

$$
q(z)=\frac{i \pi w_{0}^{2}}{\lambda}+z
$$

where $z$ is measured from the beam waist. By combining equation A.20 with equation A.17 and equating the real and imaginary parts of each one, one obtains:

$$
w^{2}(z)=w_{0}^{2}\left(1+\left(\frac{\lambda z}{\pi w_{0}^{2}}\right)^{2}\right)
$$

and

$$
R(z)=z\left(1+\left(\frac{\pi w_{0}^{2}}{\lambda z}\right)^{2}\right)
$$

which expresses the beam radius, $\omega(z)$, and the radius of curvature, $R(z)$, of the wave front in terms of the position on the $z$-axis and the beam waist.

Equation A.21 is the equation for a hyperbola with asymptote at an angle:

$$
\theta=\frac{\lambda}{\pi w_{0}}
$$

which is the far-field diffraction angle of the fundamental mode. This can be obtained by taking the limit of equation A.21 as $z \rightarrow \infty$, that is:

$$
\begin{aligned}
& \lim _{z \rightarrow \infty}\left(w^{2}(z)\right)=\lim _{z \rightarrow \infty}\left(w_{0}^{2}\left(1+\left(\frac{\lambda z}{\pi w_{0}^{2}}\right)^{2}\right)\right) \\
& \lim _{z \rightarrow \infty}\left(w^{2}(z)\right)=\lim _{z \rightarrow \infty}\left(\left(\frac{\lambda z}{\pi w_{0}}\right)^{2}\right)
\end{aligned}
$$




$$
\begin{aligned}
\frac{\lim _{z \rightarrow \infty}\left(w^{2}(z)\right)}{\lim _{z \rightarrow \infty}\left(z^{2}\right)} & =\left(\frac{\lambda}{\pi w_{0}}\right)^{2} \\
\tan ^{2} \theta & =\left(\frac{\lambda}{\pi w_{0}}\right)^{2}
\end{aligned}
$$

Using the condition that the angle of the beam is small, the approximation $\tan \theta \approx \theta$ can be used to obtain equation A.23.

Dividing equation A.22 by equation A.21 one can obtain the useful equation:

$$
\frac{\lambda z}{\pi w_{0}^{2}}=\frac{\pi w^{2}}{\lambda R}
$$

which can be used to express $w_{0}$ and $z$ in terms of $w$ and $R$, as:

$$
w_{0}^{2}=\frac{w^{2}}{1+\left(\frac{\pi w^{2}}{\lambda R}\right)^{2}}
$$

and:

$$
z=\frac{R}{1+\left(\frac{\lambda R}{\pi w^{2}}\right)^{2}} .
$$

To obtain the complex phase shift, $P(z)$, substitute the solution for $q$, equation A.20, into equation A.15 to obtain:

$$
P^{\prime}(z)=\frac{-i}{\frac{i \pi w_{2}^{2}}{\lambda}+z}
$$

which, after integration with respect to $z$, yields:

$$
\begin{aligned}
i P(z) & =\ln \left(1-\frac{i \lambda z}{\pi w_{0}^{2}}\right) \\
& =\frac{1}{2} \ln \left(1+\left(\frac{\lambda z}{\pi w_{0}^{2}}\right)^{2}\right)-i \arctan \left(\frac{\lambda z}{\pi w_{0}^{2}}\right)
\end{aligned}
$$

where the real part of $P(z)$ represent the phase shift difference, $\Phi$, between the Gaussian beam and an ideal plane wave. The imaginary part produces an amplitude factor $\frac{w_{0}}{w}$ which gives the expected intensity decrease on the axis due to the expansion of the beam. 
Substituting all of this into the original beam function, equation $A .12$ and equation A.9, gives the final form for the beam functions:

$$
u(r, z)=\frac{w_{0}}{w} \exp \left(-i(k z-\Phi)-r^{2}\left(\frac{1}{w^{2}}+\frac{i k}{2 R}\right)\right)
$$

where:

$$
\Phi=\arctan \left(\frac{\lambda z}{\pi w_{0}^{2}}\right)
$$

is the phase shift difference in between the Gaussian wave and the plane wave, and is derived from the real part of equation A.33. 


\section{APPENDIX B}

\section{CaĹl Numbers for the Bibliography}

We have taken the liberty of including the library call numbers for the books and magazines mentioned in this report, in the best interest to help an interested reader to find the references. The titles of most articles have been included for the same purpose.

1. Bulletin of the American Physical Society $\ldots \ldots \ldots \ldots \ldots \ldots$ QC 1. A56

2. Fusion Technology $\ldots \ldots \ldots \ldots \ldots \ldots \ldots \ldots \ldots \ldots \ldots$ TK 9204. N82

3. IEEE Translations on Plasma Science $\ldots \ldots \ldots \ldots \ldots \ldots \ldots$ TA 2110.I18A

4. International Journal of Infrared and Millimeter Waves ....... TA 1570.I6

5. Journal of Physics E: Scientific Instruments ............ Q 184.J6

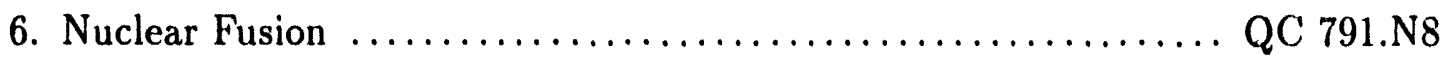

7. Plasma Physics and Controlled Fusion $\ldots \ldots \ldots \ldots \ldots \ldots$ QC 770. P5

8. Physical Review Letters $\ldots \ldots \ldots \ldots \ldots \ldots \ldots \ldots \ldots \ldots \ldots$ QC 1.P43

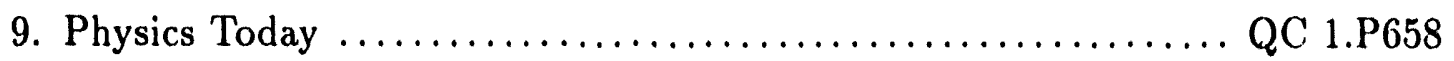

10. Radio Frequency Power in Plasmas $\ldots \ldots \ldots \ldots \ldots \ldots$ QC 718.5.115 R36X

11. Review of Scientific Instruments $\ldots \ldots \ldots \ldots \ldots \ldots \ldots \ldots \ldots$ Q 148.R5

12. Soviet Physics JETP $\ldots \ldots \ldots \ldots \ldots \ldots \ldots \ldots \ldots \ldots \ldots$ QC 1.2472 
13. Soviet Physics - Technical Physics QC 1.Z482X 


\section{BIBLIOGRAPHY}

1 Weston M. Stacey, Jr.

"Fusion

An Introduction to the Physics and Technology of Magnetic Confinement Fusion"

John Wiley \& Sons, New York, 1984

QC 791.73.S73

2 Meyerhof

"Elements of Nuclear Physics"

McGraw-Hill, New York, 1967

QC 776.M4.

3 J. G. Cordey, et al.

"Progress Toward a Tokamak Fusion Reactor"

Physics Today, Vol. 45 No. 1, p. 22, Jan. 1992

4 John W. Willis

"Cofinement Systems Program Summary"

July 1990, DOE/ER-0478T

U.S. Government Printing Office: 1990 O-862-957

5 K. G. Budden

"Radio Waves in the Ionosphere"

Cambridge U. P., Cambridge, 1961

QC 973.4.I6 B82X.

ò J. O. Thomas

Proceedings of the Institute of Radio Engineering, Vol. 47, p. 162 (1959)

7 D. Davies

"Ionospheric Radio Waves"

Chapters 3 and 8

Ginn and Blaisdell, 1969

TK 6553.D293

8 V. I. Ginzburg

"The Propagation of Electromagnetic Waves in Plasmas"

sec. 16, pp. 229-231, Series solution

sec. 17, pp. 240-242, Exact solution for linear $\epsilon$

$2^{\text {nd }}$ ed., Pergamon Press, Oxford, 1970

QC 661.G533 
9 J. F. Lyon

"The Advanced Toroidal Facility"

Fusion Tech., Vol. 10, pp. 179-226, Sept. 1986

10 Fritz Rau

"Ninth International Workshop on Stellarators"

Stellarator News, Issue \#28, July 1993

Published by Fusion Energy Division, Oak Ridge National Laboratory

Building 9201-2, P.O. Box 2009, Oak Ridge, TN 37831-8071, USA

Editor: James A. Rome, Phone: (615) 574-1306

E-Mail: JAR@ORNL.GOV

11 B. A. Carreras, et al.

"Progress in Stellarator/Heliotron Research: 1981-1986"

Nuclear Fusion, Vol. 28, No. 9, pp. 1613-1694 (1988)

12 K. M. Young, et al.

"TFTR Initial Operations"

Plasma Physics and Controlled Fusion, Vol. 26, No. 1A, pp. 11-22 (1984)

13 K. V. Orlinskij and G. Magyar

"Plasma Diagnostics on Large Tokamaks"

Nuclear Fusion, Vol. 28(4), pp. 611-697 (1988)

14 Earl C. Tanner

"Project Matterhorn: an Informal History"

Princeton Plasma Physics Laboratory Publications, 1982

QC 718.615.U62 P747

15 Earl C. Tanner

"First Princeton Tokamaks: an Informal History"

Princeton Plasma Physics Laboratory Publications, 1982

QC 718.615.U62 P7474

16 Earl C. Ta..ner

"Model C Decade: an Informal History"

Princeton Plasma Physics Laboratory Publications, 1980

QC 718.615.U62 P7475

17 A. I. Anisimov, et al.

"Method of Investigating Electron Spatial Distribution in a Plasma"

Soviet Physics - Tech. Phy. Vol. 5, No. 9, pp. 939-948, March 1961 
18 V. A. Anoshkin, et al.

"Microwave Investigations of Plasma on the 'Alpha' Device"

Soviet Physics - Tech. Phy. Vol. 5, No. 12, pp. 1370-1377, June 1961

19 R. J. Colchin

Intra-Laboratory Correspondence

ORMAK Téchnical Memo No. 105

Oak Ridge National Laboratory, May 3, 1973

20 R. J. Colchin

Intra-Laboratory Correspondence

ORMAK Technical Memo No. 93

Oak Ridge National Laboratory, January 17, 1973

21 F. Simonet

"Measurement of Electron Density Profile by Microwave Reflectometry on Tokamaks"

Review of Scientific Instruments, Vol. 56(5), pp. 664-669, May 1985

22 F. Simonet

Ph.D. Thesis

Univesité de Nancy, 1985

23 TFR Group, presented by F. Simonet

"Measurements of Electron Density Profile and Local Density Fluctuations by Microwave Reflectometry on TFR"

Proceedings of the $11^{\text {th }}$ European Conference on Controlled Fusion and Plasma Physics, Aachen, 1983

pp. 85-88, Vol. 7D, Part I

24 S. H. Heijnen, et al.

"A Multichannel Reflectometer for the RTP Tokamak"

$19^{\text {th }}$ EPS Conference on Confined Fusion and Plasma Physics

pp. 1059-1062, June 1992

25 M. A. G. Calderon and F. Simonet

"Experimental Study of a Swept Reflectometer with a Single Antenna for Plasma Density Profile Measurement"

Int. J. Infrared and Millimeter Waves, Vol. 6, No. 7, pp. 605-628 (1985)

26 J. Sánchez, et al.

"Observation of Density Fluctuations Associated with the Resonant $q=2$ 
Surface on the Wendelstein VII-AS Stellarator"

Nuclear Fusion, Vol. 30, No. 11, pp. 2383-2391 (1990)

27 J. Sánchez, et al.

"Radial Distribution of Turbulence Correlation Lengths Obtained by Reflectometry in the TJ-1 Tokamak"

$9^{\text {th }}$ Topical Conference on High-Temperature Plasma Diagnostics

March 15-19, 1992

and/or

Review of Scientific Instruments, Vol. 63(10), p. 4657, Oct. 1992

$28 \mathrm{~J}$. Sánchez, et al.

"Amplitude Modulation Reflectometry for Large Fusion Devices"

$9^{\text {th }}$ Topical Conference on High-Temperature Plasma Diagnostics

March 15-19, 1992

and/or

Review of Scientific Instruments, Vol. 63(10), pp. 4654-4656, Oct. 1992

29 J. Sánchez, et al.

Nuclear Fusion, Vol. 30, p. 2383 (1990)

30 J. Sánchez, et al.

"Reflectometry Observations of Density Fluctuations in Wendelstein VII-AS Stellarator"

Proceedings of the $17^{\text {th }}$ European Conference on Controlled Fusion and Plasma Physics, Amsterdam, 1990

pp. 1572-1575, Vol. 14B, Part VI

31 J. Sánchez, et al.

"Broadband Microwave Reflectometry on Wendelstein VII-AS Stellarator"

Proceedings of the $16^{\text {th }}$ European Conference on Controlled Fusion and Plasma Physics, Venice, 1989

pp. 1565-1568, Vol. 13B, Part VI

32 J. Sánchez, et al.

"Amplitude Modulation Reflectometry for Large Fusion Devices"

$19^{\text {th }}$ EPS Conference on Confined Fusion and Plasma Physics

pp. 1075-1078, June 1992

$33 \mathrm{~J}$. B. Wilgen, et al.

"An AM Reflectometer on PBX-M for Edge Density Profile Measurements" Bulletin of the American Physical Society, Vol. 38(6), Nov. 1993 
34 I. H. Hutchinson

"One-Dimensional Full-Wave Analysis of Reflectometry Sensitivity and Correlations"

Plasma Physics and Controlled Fusion, Vol. 34, No. 7, pp. 1225-1251 (1992)

35 I. Stakgold

"Green's Functions and Boundary Value Problems"

John Wiley \& Sons, New York (1979)

36 M. L. V. Pitteway

Proceedings of the Royal Society, Vol. 254, p. 86 (1959)

37 E. Mazzucato

Princeton Plasma Physics Laboratory

Report No. MATT-1151, Sept. 1975

38 E. Mazzucato, et al.

"TFTR Microwave Reflectometer"

$9^{\text {th }}$ Topical Conference on High-Temperature Plasma Diagnostics

March 15-19, 1992

and/or

Review of Scientific Instruments, Vol. 63(10), p. 4657, Oct. 1992

39 J. B. Wilgen, et al.

"Microwave Reflectometry for ICRF Coupling Studies on TFTR"

Princeton Plasma Physics Laboratory Report

PPPL-2915, Publically available from:

National Technical Information Service

Department of Commerce

5285 Port Royal Road

Springfield, Virginia 22161

40 G. R. Hanson, et al.

"A Swept Frequency Amplitude Modulated Reflectometer for Measuring the Edge Density Gradient on TFTR"

$9^{\text {th }}$ Topical Conference on High-Temperature Plasma Diagnostics

March 15-19, 1992

and/or

Review of Scientific Instruments, Vol. 63(10), pp. 4658-4660, Oct. 1992

41 C. Domier, et al.

"Development of Technology and Techniques for Reflectometry" 
$9^{\text {th }}$ Topical Conference on High-Temperature Plasma Diagnostics

March 15-19, 1992

and/or

Review of Scientific Instruments, Vol. 63(10), pp. 4666-4668, Oct. 1992

42 S. M. Hamberger, et al.

Physics Review Letters, Vol. 37, p. 1345 (1976)

43 G. Bottollier-Curtet and G. Ichtchenko

"Microwave Reflectometry with the Extraordinary Mode on Tokamaks: Determination of the Electron Density Profile on Petula-B"

Review of Scientific Instruments, Vol. 58(4), pp. 539-546, April 1987

44 G. Ichtchenko, et al.

"Density Profile From Microwave Reflectometry and Confinement Properties of a Lower Hybrid Current Drive Sustained Tokamak Discharge"

Proceedings of the $13^{\text {th }}$ European Conference on Controlled Fusion and Plasma Heating, 1986

pp. 419-422, Vol. 10C, Part I

45 R. N. Ó. Dubhghaill, et al.

"The Characterization of High-Speed Swept Reflectometer for Plasma Diagnostics on the RFX Reversed-Field Pinch"

International Journal of Infrared and Millimeter Waves, Vol. 13, No. 3, 1992

46 J. H. Irby and P. Stek

"Simulation of Experimental Data From the Proposed C-Mod Reflectometer"

Review of Scientific Instruments, Vol. 61(10), pp. 3052-3054, Oct. 1990

47 G. Arfken

"Mathematical Methods for Physicists"

Academic Press, New York

QA37.2 .A74 1985

Library of Congress Catalog Card Number: 73-119611

48 D. G. Swanson

"Plasma Waves"

Academic Press Inc., 1989

QC 718.5.W3S43

49 J. D. Jackson

"Classical Electrodynamics" 
John Wiley \& Sons, New York, 1975

QC 631.J3

50 K. Evenson et al.

Physics Review Letters, Vol. 29, p. 1346, 1972

51 M. B ratici

"Electron Cyclotron Emission and Absorption in Fusion Plasmas"

Nuclear Fusion, Vol. 23, No. 9, pp. 1153-1257 (1983)

52 W. P Allis

"Waves in Ánisotropic Plasmas"

Cambridge: MIT Press 1963

QC 718.A4.

53 T. H. Stix

"The Theory of Plasma Waves"

McGraw-Hill, New York, 1962

QC 718.S7

Library of Congress Catalog Number: 62-17376

54 T. H. Stix

Physics Review Letters, Vol. 15, p. 878, 1965

55 T. H. Stix

"Fast-Wave Heating of a Two-Component Plasma"

Nuclear Fusion, Vol. 15, pp. $737-754$ (1975)

56 E. T. Whittaker and G. N. Watson

"A Course of Modern Analysis"

$4^{\text {th }}$ edition, Cambridge University Press, New York, 1927

QA 401.W62

57 D. B. Batchelor, et al.

Physics of Fluids, Vol. 27, p. 2835 (1984)

$58 \mathrm{H}$. Bindslev

"Relativistic Effects in Plasma Reflectometry"

JET Report: JET-P (92) 35

59 H. Bindslev

Plasma Physics and Controlled Fusion, Vol. 33, p. 1775 (1991) 
$60 \mathrm{H}$. Bindslev

Proceedings of the International School of Plasma Physics, Piero Caldirola

p. 779, Varenna (1991)

61 H. Bindslev

Proceedings of the $18^{\text {th }}$ EPS Conference on Controlled Fusion and Plasma Physics

15C, Part IV, p. 9, Berlin (1991)

$62 \mathrm{H}$. Bindslev

"Relativistic Effects in Plasma Reflectometry"

Plasma Physics and Controlled Fusion, Vol. 34(11), pp.1601-1618 (1992)

63 N. Bretz

Private communication with Dr. Bretz.

64 B. A. Trubnikov

Plasma Physics and The problem of Controlled Thermonuclear Reactions, Vol.

3, p. 122, Pergamon Press (1959)

65 I. P. Shkarofsky

Journal of Plasma Physics, Vol. 35, p. 319 (1986)

66 P. H. Bevington

"Data Reduction and Error Analysis for the Physical Sciences"

McGraw-Hill Inc., New York, 1969

QA278 .B48 (1992)

Library of Congress Catalog Number: 69-16942

67 E. Anabitarte, E. G. Bustamante, et al.

"Characterization of a Q-Band Microwave Reflectometer for Plasma Diagnosis in the Tokamak TJ-1"

Int. J. Infrared and Millimeter Waves, Vol. 8, No. 7, pp. 733-751 (1987)

68 E. Anabitarte, et al.

"Determination of the Electron Density Profile and its Fluctuation by Broad Band Microwave Reflectometry in the TJ-1 Tokamak"

Journal of Physics D: Applied Physics

Vol. 21, pp. 1384-1390, March 1988

IOP Publishing Ltd., March 1988, pp. 1384-1390

69 A. E. Costley, et al.

"Recent Developments in Microwave Reflectometry at JET"

Review Scientific Instruments, Vol. 61(10), pp. 2823-2828, Oct. 1990 
70 A. E. Costley

Proc. Course and Workshop, Basic and Advanced Diagnostic Techniques for Fusion Plasmas

EUR 10797, Varenna, Vol. 2, pp. 379-396 (1986)

71 Mase, et al.

"Measurement of Alfvén Ion-Cyclotron Wave Using Both X-and O-Mode Reflectometers"

Physics of Fluids B, Vol. 5(6), pp. 1677-1680, June 1993

72 F. Engelmann and M. Curatolo

Nuclear Fusion, Vol. 13, p. 497 (1973)

73 I. Fidone and G. Granata

"Propagation of Electromagnetic Waves in a Plasma With a Sheared Magnetic Field"

Nuclear Fusion, Vol. 11, pp. 133-139 (1971)

74 D. A. Boyd

"What is the Limiting Polarization of Electron Cyclotron Emission Leaving a Fusion Plasma?"

$5^{\text {th }}$ Conference on Electron Cyclotron Emission, pp. 77-87, Nov. 1985, San Diego $\mathrm{Ca}$.

75 F. J. Stauffer, et al.

"Electron Cyclotron Emission Measurement on TFTR"

$5^{\text {th }}$ Conference on Electron Cyclotron Emission, pp. 18-36, Nov. 1985, San Diego $\mathrm{Ca}$.

76 TFR Group

"Local Density Fluctuations Measurements by Microwave Reflectometry on TFR"

Plasma Physics and Controlled Fusion, Vol. 27, No. 11, pp. 1299-1306 (1985)

77 TFR Group

Nuclear Fusion 18, 647 (1978)

78 M. A. Heald and C.B. Wharton

"Plasma Diagnostics with Microwaves"

New York: Wiley

QC 718.H36 
79 J. L. Doane, et al.

"Plasma Density Measurements Using FM-CW Millimeter Wave Radar Techniques"

Review Scientific Instruments, Vol. 52(1), pp. 12-15, Jan. 1981

80 A. C. C. Sips

"Reflectometry and Transport in Thermonuclear Plasmas in the Joint European Torus"

Ph.D. Thesis, Stichting voor Fundamenteel Onderzoek der Materie, 1991

81 A. C. C. Sips, et al.

"Measurements of the Electron Particle Diffusion Coefficient With the JET Multichannel Reflectometer"

Proceedings of the $16^{\text {th }}$ European Conference on Controlled Fusion and Plasma Physics, Venice, 1988

pp. 99-102, Vol. 13B, Part I

82 C. A. J. Hugenholtz

"Microwave Interferometer and Reflectometer Techniques"

Ph.D. THESIS, Stichting voor Fundamenteel Onderzoek der Materie' (FOM), 1990

83 C. A. J. Hugenholtz

"Pulse Radar Technique for Reflectometry on Thermonuclear Plasmas"

Review Scientific Instruments, Vol. 62(4), pp. 1100-1101, April 1991

84 G. R. Hanson, et al.

"ATF Two-Frequency Correlation Reflectometer"

Review Scientific Instruments, Vol. 61(10), pp. 3049-3051, Oct. 1990

85 G. R. Hanson

"Microwave Reflectometry on the Advanced Toroidal Facility to Measure Density Fluctuations and Their Radial Correlation Lengths"

Ph.D. Thesis, Georgia Institute of Technology, Atlanta Georgia, 1991

86 G. R. Hanson, et al.

"Density Fluctuation Measurement in ATF Using Correlation Reflectometry" Nuclear Fusion, Vol. 32, No. 9, pp. 1593-1608 (1992)

87 H. Jeffreys

Proc. London Mathematical Society, 23, p. 428 (1923) 
88 G. Wentzel

Z. Physik 38, p. 518 (1926)

89 H. A. Kramers

Z. Physik 39, p. 828 (1926)

90 L. Brillouin.

Compt. Rend. 183, p. 24 (1926)

91 E. Anabitarte, G. R. Hanson, et al.

"Density Fluctuation Measurements on ATF Using a Two-Frequency Reflectometer"

Procedings of the $17^{\text {th }}$ European Conference on Controlled Fusion and Plasma Physics, Amsterdam, 1990

pp. 1492-1494, Vol. 14B, Part VI

92 T. Lehecka, W. A. Peebles, et al.

"Reflectometry System for the DIII-D Tokamak"

Review of Scientific Instruments, Vol. 59(8), pp. 1620-1622, August 1988

93 T. Lehecka, et al.

"Results From the DIII-D Millimeter-wave Reflectometer"

Proceedings of the $16^{\text {th }}$ Conference on Controlled Fusion and Plasma Physics, Venice, 1989

pp. 123-126, Vol. 13B, Part I

94 E. J. Doyle, T. Lehecka, et al.

"X-Mode Broadband Reflectometry Density Profile Measurements on DIII-D" Review Scientific Instruments, Vol. 61(10), pp. 2896-2898, Oct. 1990

95 E. J. Doyle, et al.

"Reflectometer Density Fluctuation Measurements on DIII-D"

Rev. Sci. Inst. 61 (10), Oct. 1990, pp. 3016-3018

96 E. J. Doyle, et al.

"New Reflectometer Systems for the DIII-D Tokamak"

$9^{\text {th }}$ Topical Conference on High-Temperature Plasma Diagnostics

March 15-19, 1992

and/or

Review of Scientific Instruments, Vol. 63(10), p. 4665, Oct. 1992 
97 E. J. Doyle, et al.

"X-Mode Broadband Reflectometric Density Profile Measurements on DIII-D" Proceedings of the $17^{\text {th }}$ European Conference on Controlled Fusion and Plasma Heating, Amsterdam, 1990

pp. 1596-1599, Vol. 14B, Part VI

98 E. Mazzucatd and R. Nazikian

"Microwave Reflectometry for the Study of Density Fluctuations in Tokamak Plasmas"

Plasma Physics and Controlled Fusion, Vol. 33, No. 3, pp. 261-274 (1991), Great Britain

99 E. Mazzucato

"Relativistic Effects on Microwave Reflectometry"

Physics of Fluids B, Vol. 4(10), pp. 3460-3461, Oct. 1992

100 E. Mazzucato, et al.

"The Application of X-mode Reflectometry to the Study of Large Scale Fluctuations in TFTR"

$19^{\text {th }}$ EPS Conference on Confined Fusion and Plasma Physics

pp. 1055-1058, June 1992

101 R. Nazikian, et al.

"Density Fluctuation Measurements from Microwave Scattering on TFTR"

Proceedings of the $17^{\text {th }}$ European Conference in Controlled Fusion and Plasma Physics, Amsterdam, 1990

pp. 1544-1547, Vol. 14B, Part VI

102 R. Nazikian

"Modulational Sources, Sideband Correlations and Non-Stationary Interference for Waves Scattered From Random Media"

Review of Scientific Instruments, Vol. 63(11), pp. 5326-5331, Nov. 1992

103 P. Cripwell, A. E. Costly and A. E. Hubbard

"Correlation Reflectometry"

Proceedings of the $16^{\text {th }}$ European Conference on Controlled Fusion and Plasma Physics, Venice, 1989

pp. 75-78, Vol. 13B, Part I

104 T. Estrada, et al.

Review Scientific Instruments, Vol. 61, p. 3034 (1992) 
$105 \mathrm{~N}$. Bretz, et al.

"X-Mode Scattering for the Measurement of Density Fluctuations on TFTR" Review Scientific Instruments, Vol. 59(8), pp. 1538-1543, A ugust 1988

$106 \mathrm{~N}$. Bretz

"The Interpretation of Reflectometry Measurements of Plasma Fluctuations"

Princeton Plasma Physics Laboratory

Princeton, NJ 08543

107 N. Bretz

"Intrumental Aspects of Extraordinary Mode Scattering on TFTR"

Review Scientific Instruments, Vol. 61(10), pp. 3031-3033, Oct. 1990

108 N. Bretz

"One-Dimesional Modeling of the Wavelength Sensitivity, Localization, and Correlation in Reflectometry Measurements of Plasma Fluctuations"

Physics of Fluids B, Vol. 4(8), pp. 2414-2422, Aug. 1992

109 R. J. Fonck, et al.

"Long-Wavelength Density Turbulence in the TFTR Tokamak"

Physical Review Letters, Vol. 70(24), pp. 3736-3739, June 1993

110 R. J. Groebner, et al.

"Role of Edge Electric Field and Poloidal Rotation in the L-H Transition"

Physical Review Letters, Vol. 64(25), pp. 3015-3018, June 1990

111 S. F. Paul, et al.

"Measurements of Long-Wavelength Density Fluctuations in TFTR"

Physics of Fluids B, Vol. 4(9), pp. 2922-2928, Sept. 1992

112 I. Collazo-López

Fortran computer program on the ORNL Fusion Energy Division Vax Cluster. Available upon request. E-Mail: COLLAZOI@FEDC06.FED.ORNL.GOV

113 L. Vahala, et al.

"Measurement of Magnetic Fluctuations by $O \rightarrow X$ Mode Conversion"

Review Scientific Instruments, Vol. 61 (10), pp. 3022-3024, Oct. 1990

114 L. Vahala, et al.

"Electromagnetic Wave Scattering from Magnetic Fluctuations in Tokamaks" Physics of Fluids B, Vol. 4(3), pp. 619-629, March 1992 
115 G. D. Conway

"Scattering of Reflectometer Signals from Rippled Surfaces"

Accepted for publication by Review of Scientific Instruments

Prepared January 1993, revised March 1993.

116 J. P. García, et al.

"Scattering Effects of Small-Scale Density Fluctuations on Reflectometric Measurements in a Tokamak Plasma"

Proceedings of the $16^{\text {th }}$ European Conference on Controlled Fusion and Plasma Physics, Venice, 1989

pp. 1521-1524, Vol. 13B, Part VI

117 A. Hubbard, D. Ward and T. E. Stringer

"Direct Measurement of the Electron Diffusion Coefficient on JET Using a Microwave Reflectometer"

Proccedings of the $13^{\text {th }}$ European Conference on Controlled Fusion and Plasma Heating, Schliersee, 1986

pp. 232-235, Vol. 10C, Part I

118 Paul Michelsen

"Simulation of Correlation Reflectometry"

$19^{\text {th }}$ EPS Conference on Confined Fusion and Plasma Physics

pp. 1067-1070, June 1992

119 L. Rhodes, et al.

"The UCLA Correlation Reflectometer System on DIII-D"

$9^{\text {th }}$ Topical Conference on High-Temperature Plasma Diagnostics

March 15-19, 1992

and/or

Review of Scientific Instruments, Vol. 63(10), pp. 4661-4664, Oct. 1992

120 T. L. Rhodes, et al.

"Microwave Reflectometry as a Density Fluctuation Diagnostic: a Comparison Between a Controlled Laboratory Experiment and a One Dimensional Numerical Model"

$19^{\text {th }}$ EPS Conference on Confined Fusion and Plasma Physics

pp. 1071-1073, June 1992

121 H. J. Hartfuss

"Broadband Heterodyne Reflectometry on the W7-AS Stellarator" $9^{\text {th }}$ Topical Conference on High-Temperature Plasma Diagnostics March 15-19, 1992 
and/or

Review of Scientific Instruments, Vol. 63(10), p. 4653, Oct. 1992

122 S. Martini, et al.

"Interferometry and Reflectometry Diagnostics for RFX"

Proceedings of the $16^{\text {th }}$ European Conference on Controlled Fusion and Plasma Physics, Veniice, 1989

pp. 1493-1496, Vol. 13B, Part VI

123 Anatoly Skibenko and Rostilav Pavlichenko

"Electron Density Profile Reconstruction for the Uragan-3M Torsatron Via Upper X-Mode reflectometry"

Stellarator News, Issue \#25, January 1993

Published by the ORNL Fusion Energy Division

Building 9201-2, P.O. Box 2009

Oak Ridge, TN 37831-8071

Editor: James A. Rome

E-Mail: JAR@ORNL.GOV

124 M. E. Manso, et al.

"Broadband Microwave Reflectometry on ASDEX"

Proceedings of the $16^{\text {th }}$ European Conference on Controlled Fusion and Plasma Physics, Venice, 1989

pp. 1517-1520, Vol. 13B, Part VI

125 M. E. Manso, et al.

"Localized Density Measurements on ASDEX Using Microwave Reflectometry" Proceedings of the $17^{\text {th }}$ European Conference on Controlled Fusion and Plasma Physics, Amsterdam, 1990

pp. 1560-1563, Vol. 14B, Part VI

126 R. Schubert, et al.

"Edge Density X-Mode Reflectometry of RF-Heated Plasmas on ASDEX"

Proceedings of the $17^{\text {th }}$ European Conference on Controlled Fusion and Plasma Physics, Amsterdam, 1990

pp. 1552-1555, Vol. 14B, Part VI

127 A. Silva, et al.

"A Microwave Reflectometric System for Tokamak ASDEX Upgrade" $19^{\text {th }}$ EPS Conference on Confined Fusion and Plasma Physics

pp. 1063-1066, June 1992 
128 F. Serra, et al.

"Reflectometry Measurements of the $m=1$ Satellite Mode in L- and H-mode Plasmas in ASDEX"

$19^{\text {th }}$ EPS Conference on Confined Fusion and Plasma Physics

pp. 231-234, June 1992

129 P. Millot, et al.

Proceedings of the $14^{\text {th }}$ European Conference on Controlled Fusion and Plasma Physics, Madrid, Spain

pp. 1303-1304, Vol. 11D, Part III (1987)

130 W. A. Peebles, et al.

"Fluctuation Measurements in the DIII-D and TEXT Tokamaks Via Collective Scattering and Reflectometry"

Review of Scientific Instruments 61 (11), pp. 3509-3519, Nov. 1990

131 T. Fukuda, et al.

"Broadband Reflectometry for the Density Profile and Fluctuation Measurements in the JT-60 Tokamak"

Review of Scientific Instruments, Vol. 61(11), pp. 3524-3527, Nov. 1990

132 H. Meuth and E. Sevillano

"Microwave Plasma Diagnostics"

PFC/JA-87-42

133 V. E. Golant

Zh. Tekh. Fiz., Vol. 30, p. 1261, 1960

Soviet Physics - Technical Physics, Vol. 5, p. 1240 (1961)

134 V. A. Vershkov and V. A. Zhuravlev

"Plasma Diagnostics Experiments on the T-10 Tokamak by Means of a Reflected Microwave Signal"

Soviet Physics - Technical Physics, Vol. 32(5), pp.523-526 (1987)

135 O. S. Pavlichenko, et al.

ITER Diagnostic Workshop, Garching, Germany

ITER-IL-Ph-07-0-15, Feb. 12-23 (1990)

136 A. E. Hubbard, et al.

"A Simple Fixed-Frequency Reflectometer for Plasma Density Profile Measurements on JET"

Journal of Physics E: Scientific Instruments, 20 pp. 423-427 (1987) 
137 A. E. Hubbard

"Measurement of Electron Density on JET by Microwave Reflectometry"

$\mathrm{Ph}$. D. Thesis, May 1987

University of London

Imperial College of Science and Technology

138 K. Tomabechi

Proceedings of the $12^{\text {th }}$ International Conference on Plasma Physics and Controlled Nuclear Fusion Research, NICE (1988)

pp. 215, Vol. 3

139 M. Abramowitz and I. A. Stegun

"Handbook of Mathematical Functions"

$5^{\text {th }}$ ed., DOVER, New York, 1968

C 13.32:55/2

140 R. Cano and C. Cavallo

Proceedings of the $5^{\text {th }}$ International Conference on Infrared and Millimeter Waves

Würtzburg, 1980

141 M. Brambilla and M. Moresco

"The Influence of Mode Mixing on Reflectometry Density Measurements in a Reversed Field Pinch"

Plasma Physics and Controlled Fusion, Vol. 29, No. 3, pp. 381-393 (1987)

142 J. Lohr

"Electron Density Measurements From Cutoff of Electron Emission in the DIII-D Tokamak"

Review Scientific Instruments, Vol. 59(8), pp. 1608-1610, Aug. 1988

143 J. Adam, et al.

"Wave Generation and Heating in the ST-Tokamak at the Fundamental and Harmonic Cyclotron Frequencies"

Plasma Physics and Controlled Nuclear Fusion Research

Vol. 1, IAEA, Vienna (1975)

144 J. C. Hosea, et al.

"Fast Wave Heating in the Princeton Large Torus"

Princeton Plasma Physics Laboratory Report-1588, October 1979 
145 TFR Group

"Impurity Pump-Out at the Two Ion Hybrid Resonance During ICRF Experiments in TFR Tokamak Plasmas"

EUR-CEA-FC-1131, October 1981

146 R. J. Taylor, et al.

"Observation' of Short ICRF Waves in Microtor/Macrotor"

$4^{\text {th }}$ Topical Conference in RF Heating, Austin, TX (1981)

147 A. P. Biddle and J.C. Sprott

"High Power Heating in the Ion Cyclotron Range of Frequencies in the Wisconsin Tokapole II"

University of Wisconsin report UC-20f, September 1980

148 A. W. Molvik, et al.

"Initial TMX Central-Cell ICRH Experiments"

Lawrance Livermore Laboratory Report UCID-18866, December 1980

149 T. Watari, et al.

"A Slow-Wave Heating Experiment on RFC-XX Using an Array of Phased Antennas"

Nuclear Fusion, Vol. 22, No. 10 (1982)

150 D. K. Smith and B. D. McVay

"Low Frequency RF Pumping of Electrons for Potential Enhancement in Tandem Mirrors"

Bulletin of the American Physical Society, Vol. 27, No. 8, Part II

151 K. Steinmetz, et al.

"Observation of a High-Confinement Regime in a Tokamak Plasma with Ion Cyclotron-Resonance Heating"

Physical Review Letters, Vol. 58, No. 2, pp. 124-127, Jan. 1987

152 N. J. Fisch

Rev. Mod. Phys., Vol. 59, p. 175 (1987)

153 N. J. Fisch

"Heating in Toroidal Plasmas"

Brussels: Commission of the European Communities

Proc. $3^{\text {th }}$ Joint Varenna-Grenoble Int. Symp. Vol. 3, p. 841

154 N. J. Fisch

Nuclear Fusion, Vol. 21, p. 15 (1981) 
155 Dave Rasmussen

Private communication

156 T. J. Wade, et al.

"High Power (22MW) ICRH at JET and Developments for Next Step Devices" $14^{\text {th }}$ IEEE/NPSS Symposium, Sept. 1991, San Diego, Ca.

pp. 902-907; 91CH3035-3 (CIEEE

157 K. Steinmetz

"ICRF Heating in Fusion Plasmas"

AIP Conferènce Proceedings 1987

Radio Frequency Power in Plasmas, pp. 211-219 (1987)

158 G. Schilling

"Overview of TFTR ICRF Results"

$10^{\text {th }}$ Topical Conference on Radio Frequency Power in Plasmas

April, 1993, Boston, Massachusetts

159 J. M. Noterdaeme

"First Results of Ion Cyclotron Resonance Heating in ASDEX Upgrade"

$10^{\text {th }}$ Topical Conference on Radio Frequency Power in Plasmas

April, 1993, Boston, Massachusetts

160 B. Saoutic

"High Power ICRH and LHCD Experiments on Tore Supra"

$10^{\text {th }}$ Topical Conference on Radio Frequency Power in Plasmas

April, 1993, Boston, Massachusetts

161 T. Fujii

"Lower Hybrid Current Drive and ICRH Heating Experiments on JT-60U"

$10^{\text {th }}$ Topical Conference on Radio Frequency Power in Plasmas

April, 1993, Boston, Massachusetts

162 DIII-D team, presented by T. C. Simonen

"Recent Results From DIII-D and Future Plans"

$14^{\text {th }}$ IEEE/NPSS Symposium on Fusion Engineering

pp. 1068-1076, Sept. 1991, San Diege, Ca.

163 D. Baker and the DIII-D group

"DIII-D Research Operations: Annual Report to the U.S. Department of Energy"

October 1, 1991 through September 30, 1992

Date Published: May 1993 
Available to $D O E$ from:

Office of Scientific and Technical Information

P.O. Box 62

Oak Ridge, TN 37831

164 R. A. Cairns

"Rádiofrequency Heating of Plasmas"

Adam Hilger, Bristol, Philadelphia and New York

QC718.5.H5C35 1991

ISBN 0-7503-0034-5

165 D. R. Baker, et al.

"Tests of an ICRH Resonant Cavity Launcher with Faraday Shield"

IEEE Translations on Plasma Science, Vol. PS-14, No. 1, pp. 53-58, Feb. 1986

166 L. K. Kuznetsova, et al.

"Electron Cyclotron Current Drive and Tearing Mode Stabilization in ITER"

EPS $17^{\text {th }}$ Conference on Controlled Fusion and Plasma Heating, Amsterdam, 1990 , pp. 1247-1250, Vol. 14B, Part III

167 F. X. Söldner, et al.

"Profile Control with Lower Hybrid Waves on ASDEX"

EPS $17^{\text {th }}$ Conference on Controlled Fusion and Plasma Heating, Amsterdam, 1990, pp. 1323-1326, Vol. 14B, Part III

168 R. H. Goulding, et al.

"Development of Fast-Wave ICRF Current Drive Systems at ORNL"

EPS $17^{\text {th }}$ Conference on Controlled Fusion and Plasma. Heating, Amsterdam, 1990, pp. 1311-1314, Vol. 14B, Part III

169 J. Adam

"Review of Tokamak Plasma Heating by Wave Damping in the Ion Cyclotron Range of Frequency"

Plasma Physics and Controlled Fusion, Vol. 29, pp. 443-472 (1987)

170 D. M. Manos, et al.

"Studies of the Edge Plasma of RF Heated PLT Discharges"

Journal of Nuclear Materials, Vol. 129, pp. 319-324 (1985)

171 J. R. Wilson, et al.

"The Evolution of Plasma Parameters as Governed by Edge Phenomena During Ion Bernstein Wave Heating"

Journal of Nuclear Materials, Vol. 145-147, pp. 161-620 (1987) 
172 C. E. Thomas, et al.

"ICH/Edge Interactions on TORE SUPRA: First Data"

Oral presentation on Tuesday, 5 January 1993

Fusion Energy Division, ORNL

173 N. Noda, et al.

"Impurity Origin During ICRF Heating in the JIPF: T-IIU Tokamak"

Journal of Nuclear Materials, Vol. 128-129, pp. 304-309 (1984)

174 H. Tamai, et al.

"Edge Plasma Characteristics During ICRF Heating on JFT-2M"

Fusion Engineering and Design, Vol. 12, pp. 25-29 (1990)

175 L. I. Grigoreva, et al.

"A Study of the Mechanics of Metal Impurity Release During ICRF Heating in the URAGAN-3 Torsatron"

Journal of Nuclear Materials, Vol. 162-164, pp. 458-461 (1989)

176 E. D. Volkov, et al.

"On the Mechanisms of Light and Heavy Impurity Release During RF Plasma Heating in the URAGAN-3 Torsatron"

Fusion Engineering and Design, Vol. 12, p. 237 (1990)

177 H. L. Manning, et al.

"Impurity Generation During ICRF Heating Experiments on Alcator C"

Nuclear Fusion, Vol. 26, pp. 1665-1678 (1986)

178 A. S. Wan, et al.

"The Effects of ICRF on the Alcator C Scrape-off Layer Plasma"

Journal of Nuclear Materials, Vol. 162-164, pp. 292-299

179 C. Janeschitz, et al.

"Impurity Production During ICRF Heating"

Europhysics Conference Abstracts, Vol. 10C, pp. 678-681, EPS (1986)

180 B. Schweer, et al.

"Effects of ICRF Heating on the Plasma Edge in TEXTOR"

Europhysics Conference Abstracts, Vol. 10C, pp. 399-402, EPS (1985)

181 U. Samm, et al.

"Plasma Edge Physics in the TEXTOR Tokamak With Poloidal and Toroidal Limiters"

Journal of Nuclear Materials, Vol. 162-164, pp. 24-27 (1989) 
182 R. Van Nieuwenhove, et al.

"Experimental Evidence for Sheath Effects at the ICRF Antenna and Ensuing Changes in the Plasma Boundary During ICRF on TEXTOR"

Journal of Nuclear Materials, Vol. 162-164, pp. 288-291 (1989)

183 R. Van Nieuwenhove, et al.

"Influence of 'ICRF on the Edge Plasma of Tokamaks"

PhD Thesis, University of Antwerp, B-2610, Antwerp (1989)

184 M. Bures, et al.

"The Modification of the Plasma Edge and Impurity Production by Antenna Phasing During ICRF Heating on JET"

Plasma Physics and Controlled Fusion, Vol. 30, pp. 149-167 (1988)

185 J. A. Tagle, et al.

"The Effect of Different ICRF Heating Scenarios on the JET Scrap-Off Layer" Journal of Nuclear Materials, Vol. 162-164, pp. 282-287 (1989)

186 T. Fujii, et al.

"Interaction Between RF and Edge Plasma During ICRF Heating in JT-60"

Fusion Engineering and Design, Vol. 12, pp. 139-148 (1990)

187 D. B. Batchelor, et al.

"ICRF Heating"

Fusion Technology, Vol. 21, pp. 1214-1242 (1992)

188 P. P. Colestock

"Review of ICRF Experiments"

IEEE Trans. of Plasma Science, PS-12, p. 64 (1984)

189 H. Takahashi

"ICRF Heating in Tokamaks"

J. de Physique, Vol. 38, C6 (1977)

190 S. Yoshikawa

"Ion Heating in the C-Stellarator"

Plasma Physics and Controlled Nuclear Fusion Research

$2^{\text {nd }}$ International Conference, Culham 1965

Vol. 2, IAEA, p. 925 (1965)

191 J. I. Lodge and R.W. Steward

"Studies in High Frequency Discharges"

Canadian J. of Res., Vol. 26, section A, p. 205 (1948) 
192 H. S. Butler and G. S. Kino "Plasma Sheath Formation by Radio Frequency Fields" Physics of Fluids, Vol. 6, p. 1346 (1963)

193 V. L. Vdovin, et al. "Excitation of Magnetosonic Resonance in the Tokamak Plasma" JETP Letter, Vol. 14, p. 149 (1971)

194 V. L. Vdovin, et al. "Magnetosonic Plasma Heating" JETP Letter, Vol. 17, p. 2 (1973)

195 V. L. Vdovin, et al. "Investigation of the Magnetosonic Plasma Heating in the Tokamak" Controlled Fusion and Plasma Physics, $6^{\text {th }}$ European Conference, Moscow, p. 553 (1973)

196 V. L. Vdovin, et al.

"Plasma Heating with an Azimuthally Non-Symmetrical Ion Cyclotron Wave in Tokamak TM-1-HF"

Plasma Physics and Controlled Nuclear Fusion Research $5^{\text {th }}$ IAEA Conference, Tokyo, 1974

Nuclear Fusion Suppl. IAEA, English translation of the Russian paper, p. 21 (1975)

197 J. C. Hosea, et al.

"Ion Cyclotron and Fast Hydromagnetic Wave Generation in the ST Tokamak" Physics Review Letters, Vol. 31, p. 150 (1973)

198 J. Adam, et al.

"Wave Generation and Heating in the ST-Tokamak at the Fundamental and Harmonic Ion Cyclotron Frequencies"

Plasma Physics and Controlled Nuclear Fusion Research

$5^{\text {th }}$ IAEA Conference, Tokyo, 1974, Vol. 1, IAEA 65

199 J. C. Hosea

"Fast and Slow Ion Cyclotron Wave Generation and Heating in the ST Tokamak"

Symp., Varenna, 1974, CNR, Milano, p. 61 (1973)

200 TFR Group

"TFR Experiments on Superbanana Particle Diffusion, Small Scale Turbulence and Transport" 
Plasma Physics and Controlled Nuclear Fusion Research $8^{\text {th }}$ IAEA Conference, Brussels, 1980, IAEA, p. 425 (1981)

201 TFR Group "ICRF Heating in TFR and the Problem of Impurity Release" Plasma Physics and Controlled Fusion, Vol. 26, p. 165 (1984)

202 TFR Group

"Plasma Edge Measurements During Additional Heating in the TFR Tokamak" Plasma Physics and Controlled Fusion $11^{\text {th }}$ EPS Conference, Aachen (1983)

Europhysics Conference Abstracts, Vol. 7D(II), EPS, p. 493 (1983)

203 TFR Group "ICRF Results on TFR at Megawatt Power Levels" Plasma Physics, Vol. 24, p. 615 (1981)

204 TFR Group "ICRF Heating in TFR" CEA, DRFC-SCP EUR-CEA-FC-1186, June 1983

205 TFR Group "Graphite Limiter Used in TFR"

Journal of Nuclear Materials, Vol. 128-129, p. 292 (1984)

206 TFR Group and Sand F. "ICRF Heating Experiments in TFR Using a Low-Field-Side Antenna" Nuclear Fusion, Vol. 25, p. 1719 (1985)

207 J. C. Hosea, et al.

"Fast Wave Heating in the Princeton Large Torus"

Princeton University, NJ(USA)

Princeton Plasma Physics Lab., Experimental Report PPPL-1588, Oct .1979

208 P. L. Colestock, et al.

"The Effects of ICRF Heating on Plasma Edge Conditions in PLT"

Journal of Vacuum Science and Technology, A. Vol. 3, pp. 1211-1217 (1985)

209 H. Ogawa, et al.

"Impurity Behaviour During ICRF Heating in JFT-2M"

Journal of Nuclear Materials, Vol. 128-129, p. 298 (1984) 
210 S. I. Itoh, et al.

"Three-dimensional Study of Coupling Efficiency of ICRF Wave Heating. Antenna Launcher and Wave Guide Launcher"

Heating in Toroidal Plasmas, $4^{\text {th }}$ International Symposium, Rome (1984)

Vol. 1, p. 407, Nuclear Energy Agency (1984)

211 K. Itoh, et al.

"A Possible Origin of Metal Impurities in ICRF Heating Experiments"

Comments Plasma Physics and Controlled Fusion, Vol. 10, p. 91 (1986)

212 M. Mori, et al.

"High-Power ICRF Heating Experiments in the JFT-2M Tokamak"

Plasma Physics and Controlled Nuclear Fusion Research

$10^{\text {th }}$ IAEA International Conference, London, Vol. 1, p. 445, IAEA (1984)

213 H. Tamai, et al.

"Impurity Reduction During ICRF Heating in JFT-2M Tokamak"

Nuclear Fusion, Vol. 26, p. 365 (1986)

214 H. Tamai, et al.

"Supression of the Metal Impurity Release During ICRF Heating by the Installation of Carbon Blocks"

Japan Atomic Energy Research Institute, Tokyo

Experimental Report JAERI-M-86-143, Oct. 1986

215 M. Bures, et al.

"Role of the Antenna Screen Angle During ICRF Heating in JET"

Nuclear Fusion, Vcl. 30, p. 251 (1990)

216 M. Brambilla, et al.

"Theoretical and Experimental Investigation of a Mechanism for Impurity Production by ICRF Fields"

Plasma Physics and Controlled Nuclear Fusion Research

$13^{\text {th }}$ IAEA Conference, Washington, 1990

Vol. 1, p. 723, IAEA, VIenna (1991)

217 M. Brambilla, et al.

"Introduction of Parallel Electric Fields at the Plasma Edge During ICRF Heating"

Controlled Fusion and Plasma Heating

$17^{\text {th }}$ EPS Conference, Amsterdam, 1990

Vol. 14B(III), p. 1056, EPS (1990) 
218 F. W. Perkins

Princeton Plasma Physics Laboratory Report No. 2571, Princeton (1988)

219 F. W. Perkins

"Radiofrequency Sheaths and Impurity Generation by ICRF Antennas"

Nuclear Fusion, Vol. 29, No. 4, pp. 583-592 (1989)

220 J. R. Myra, et al.

Physics of Fluids B1, Vol. 6, p. 1193 (1989)

221 J. B. O. Caughman II, et al.

"Experimental Evidence of Increased Electron Temperature, Plasma Potential, and Ion Energy Near an ICRF Antenna Faraday Shield"

Fusion Engineering and Design, Vol. 12, pp. 179-183 (1990)

222 J. B. O. Caughman

"The Distribution of Ion Energies Incident on a ICRH Antenna Faraday Shield" PhD Thesis, University of Illinois, Urbana, Il. (1989)

223 I. S. Lehrman

"A Study of Coupling and Edge Processes for ICRF Antennas"

PhD Thesis, University of Wisconsin, Madison, Wi. (1988)

224 J. M. Noterdaeme, et al.

"Experimental Results on Edge Effects During ICRF Heating of ASDEX Plasmas"

Fusion Engineering and Design, Vol. 12, pp. 127-137 (1990)

225 M. D. Carter, et al.

"Electron Heating and Static Sheath Enhancement in Front of Energized RF Antennas"

Fusion Engineering and Design, Vol. 12, pp. 105-110 (1990)

226 D. A. D'Ippolito, et al.

"Impurity Release by ICRF Antennas in JET"

Fusion Engineering and Design, Vol. 12, pp. 209-216 (1990)

227 R. J. Taylor, et al.

"Particle Transport Due to ICRF Waves and Radial Electric Fields in Tokamaks"

Plasma Physics and Controlled Nuclear Fusion

$9^{\text {th }}$ International Conference, Baltimore, Vol. 3, pp. 251-261, IAEA (1982) 
228 L. 1. Grigor'eva, et al.

"Plasma Potential Formation Under the Influence of an External RF Field and the Effect of this Potential on Particle Transport"

Journal of Nuclear Materials, Vol. 128-129, p. 317 (1984)

229 J. M. Noterdaeme, et al.

"Plasma Ed'ge Effects with ICRF in ASDEX"

Controlled Fusion and Plasma Physics

$14^{\text {th }}$ European Conference, Madrid, 1987

Europhysics Conference Abstracts, Vol. 11D(pt 2), p. 678, EPS, Geneva

230 R. Van Nieuwenhove, et al.

"Observation of a Localized RF Electric Field Structure in the Scrape-Off Layer During ICRF on TEXTOR and ASDEX"

European Conference on Controlled Fusion and Plasma Physics

Europhysics Conference Abstract, Vol. 13B(pt.3), p. 3, EPS, Geneva (1989)

231 R. Van Nieuwenhove, et al.

"Effect of RF Heating on D.C. Electric Fields in the SOL of TEXTOR"

Fusion Engineering and Design, Vol. 12, p. 231 (1990)

232 D. A. D'Ippolito, et al.

"ICRF-Driven Convective Cells in the Tokamak Edge Plasma"

Presented at the Sherwood Fusion Theory Conference (1991)

233 I. A. Moyer, et al.

"Effects of Auxiliary Heating on D.C. Electric Fields in the TEXTOR Boundary"

Plasma Surface Interaction

$9^{\text {th }}$ International Conference, Bournemounth (1990)

234 D. Bora, et al.

"Effects of ICRH on Electrostatic Fluctuations and Particle Transport in the Boundary Plasma of TEXTOR"

To be published in Nuclear Fusion

235 S. K. Erents, et al.

Journal of Nuclear Materials, Vol. 145-147, p. 231 (1987)

236 I. S. Lehrman, et al.

Proceedings of the $7^{\text {th }}$ APS Topical Conference on Applications of Radio Frequency Power to Plasmas, Kissimmee, Fl (1987) 
237 G. J. Greene

International Conference on Plasma Physics, Lausanne, p. 5 (1984)

238 R. Van Nieuwenhove, et al.

$8^{\text {th }}$ International Conference on Plasma Surface Interactions, Jülich (1988)

239 I. L. Lehrman, et al.

Fusion Engineering Design, Vol. 12, pp. 51-62 (1990)

240 D. J. Hoffman

"Experimental Meusurements of the Ion Cyclotron Antennas Coupling and RF Characteristics"

Fusion Technology, Vol. 8, pp. 411.419 (1985)

241 D. J. Hoffman

"The Design of High-Power ICRF Antennas for TFTR and TORE SUPRA" Applications of Radio-Frequency Power to Plasmas

Proc. $7^{\text {th }}$ Topical Conference, Kissimmee, Florida p. 302 (1987)

242 J. R. Wilson

"TFTR ICRF Antenna Design"

AIP Conference Proceedings 1987, pp. 294-297

243 J. E. Stevens

"The Effect of ICRF Antenna Phasing on Metal Impurities in TFTR" Plasma Physics and Controlled Fusion (G.B.), Vol. 32, No. 3, pp. 189-196 (1990)

244 J. E. Stevens, C. Bush and P.L. Colestock

Princeton Plasma Physics Laboratory Internal Report

PPPL-2640, Princeton (1989)

245 G. L. Chen

"Resonant Loop Antenna Design with a 2-D Steady-State Analysis"

AIP Conference Proceedings 1987, pp. 382-385

246 F. W. Baity

"Modeling of Compact Loop Antennas"

AIP Conference Proceedings 1987, pp. 378-381

247 W. L. Gardner

"ICH Antenna Development of the ORNL RF Test Facility"

AIP Conference Proceedings 1987, pp. 306-309 
248 V. L. Granatstein and P. L. Colestock

"Wave Heating and Current Drive in Plasmas"

Gordon and Breach Science Publishers

QC 718.5.H5W38 (1985)

249 A. Bers

IEEE Trans: Plasma Science, PS-12 No. 2 (1984)

Special Issue on RF Heating and Current Generation

250 J. F. King

"Materials Test and Analyses of Faraday Shield Tubes for ICRF Antennas" Fusion Technology, Vol. 15, Mar. 1989, pp. 1093-1096

251 D. J. Paylor

"Resonant Double Loop Antenna Developments at ORNL"

Fusion Technology, Vol. 15, Mar. 1989, pp. 1088-1092

$252 \mathrm{M}$. Goniche, et al.

"Lower Hybrid Wave Experiments in TORE SUPRA"

EPS $17^{\text {th }}$ Conference on Controlled Fusion and Plasma Heating, Amsterdam, 1990, pp. 1231-1234, Vol. 14B, Part III

253 M. Kwon, et al.

"Measurement of the Effects of Faraday Shields on ICRH Antenna Coupling"

IEEE Translations on Plasma Science, Vol. 18, No. 2, pp. 184-189, April 1990

254 B. D. Blackwell

"The Alcator-C ICRF Experiment"

Bulletin of the American Physical Society, 29, p. 1219 (1984)

255 J. B. O. Caughman II

"Measurements of the Transmission Characteristics of the Alcator-C ICRH Antenna"

Bulletin of the American Physical Society, 29, p. 1221 (1984)

256 McDonnell Douglas Co.

"Technical Proposal for TFTR/Big Dee ICRH Launcher Development"

Vol. 1, MDC E2675, McDonnell Douglas Corporation (1983)

257 R. H. Dicke

"Molecular Amplification and Generation Systems and Methods"

U.S. Patent 2,851,652 - Sept. 9, 1958 
258 A. M. Prokhorov "Molecular Amplifier and Generator for Sub-Millimeter Waves" JETP (USSR), Vol. 34, pp.1658-1659, June 1959

Soviet Physics JETP, Vol. 7, pp. 1140-1141, Dec. 1958

259 A. L. Schawlow and C. H. Townes "Infrared and Optical Masers" Physics Review, Vol.29 pp. 1940-1949, Dec. 1958

260 A. G. Fox and T. $\mathrm{Li}$ "Resonant Modes in an Optical Maser" Proc. IRE (Correspondence), Vol. 48, pp. 1904-1905, Nov. 1960

261 A. G. Fox and T. Li "Resonant Modes in a Maser Interferometer"

Bell Sys. Tech. J., Vol. 40, pp. 453-488, March 1961

262 G. D. Boyd and J. P. Gordon "Confocal Multi-Mode Resonator for Millimeter Through Optical Wavelength Lasers"

Bell Sys. Tech. J., Vol. 40, pp. 489-508, March 1961

263 G. D. Boyd and H. Kogelnik

"Generalized Confocal Resonator Theory"

Bell Sys. Tech. J., Vol. 41, pp. 1347-1369, July 1962

264 G. Goubau and F. Schwering

"On the Guided Propagation of Electromagnetic Wave Beams"

IRE Trans. on Antennas and Propagation, Vol. AP-9, pp. 248-256, May1961

265 J. R. Pierce

"Modes in Sequences of Lenses"

Proc. NAT'L Acad. Sci., Vol. 47, pp. 1808-1813, Nov. 1961

266 J. R. Pierce

"Theory and Design of Electron Beams"

New York, Van Nostrand, 1954, p. 194

TK 7815.P5

267 G. Gaubau

"Optical Relations for Coherent Wave Beame

Electromagnetic Theory and Antennas"

New York, MacMillan, 1963, pp. 907-918 
268 H. Kogelnik "Imaging of Optical Mode-Resonators With Internal Lenses"

Bell Sys. Tech. J., Vol. 44, pp. 455-494, March 1965

269 H. Kogelnik, et al.

"On the Propagation of Gaussian Beams of Light Through Lens-Like Media Including Those With a Loss or Gain Variation" Applied Optics, Vol. 4, pp. 1562-1569, Dec. 1965

270 A. E. Siegman

"Unstable Optical Resonators for Laser Applications"

Proceedings IEEE, Vol. 53, pp. 277-287, March 1965

271 W. Brouwer

"Matrix Methods in Optical Instruments Design"

New York, Benjamin, 1964

QC 371.B847

272 E. L. O'Neill

"Introduction to Statistical Optics"

Mass.,Addison-Wesley, 1963

273 C. S. Williams

"Gaussian Beam Formulas From Diffraction Theory"

Applied Optics, Vol. 12, No. 4, pp. 872-876, April 1973

274 T. S. Bigelow

"Design of a Reflectometer System for Measurement of the Plasma Edge Density Profile on TFTR"

APS Conference, Nov. 1992, Seattle, Washington

275 I. Collazo-López

"Preliminary Results of the ORNL Swept Two-Frequency X-Mode Reflectometer for TFTR"

APS Conference, Nov. 1992, Seattle, Washington

276 S. Raftopoulos, et al.

"Design of ICRF antennas for TFTR"

$14^{\text {th }}$ IEEE/NPSS Symposium, Sept. 1991, San Diego, Ca.

pp. 111-114, 91CH3035-3 C.IEEE 
277 M. McCarthy, et al.

"TFTR Microwave Reflectometer Diagnostic"

$14^{\text {th }}$ IEEE/NPSS Symposium, Sept. 1991, San Diego, Ca.

pp. 1192-1194, 91CH3035-3 (CIEEE

$278 \mathrm{H}$. Kogelnik and $\mathrm{T}$. Li

"Laser Beams and Resonators"

Proceedings of the IEEE, Vol. 54, No. 10, pp. 1312-1329, Oct. 1966

279 Born and Wolf

"Pricinples of Optics"

Pergamon Press, New York, 1959

QC 355.B63

280 David M. Pozar

"Microwave Engineering"

Addison-Wesley Publishing Company, New York, 1990

TK 7876.P69

281 J. T. Coleman

"Microwave Devices"

Reston Publishing Company, Inc.

Reston, Virginia 22090

TK7876.C64

ISBN 0-8359-4386-0

282 O. P. Gandhi

"Microwave Engineering and Applications"

Pergamon Press, New York

TK 7876-G36

ISBN 0-08-025589-2 
ACKNOWLEDGEMENT

$$
\checkmark
$$

This work was supported by Martin Marietta Energy systems under Subcontract 19X-SJ664C with the Georgia Tech Research Corporation. 


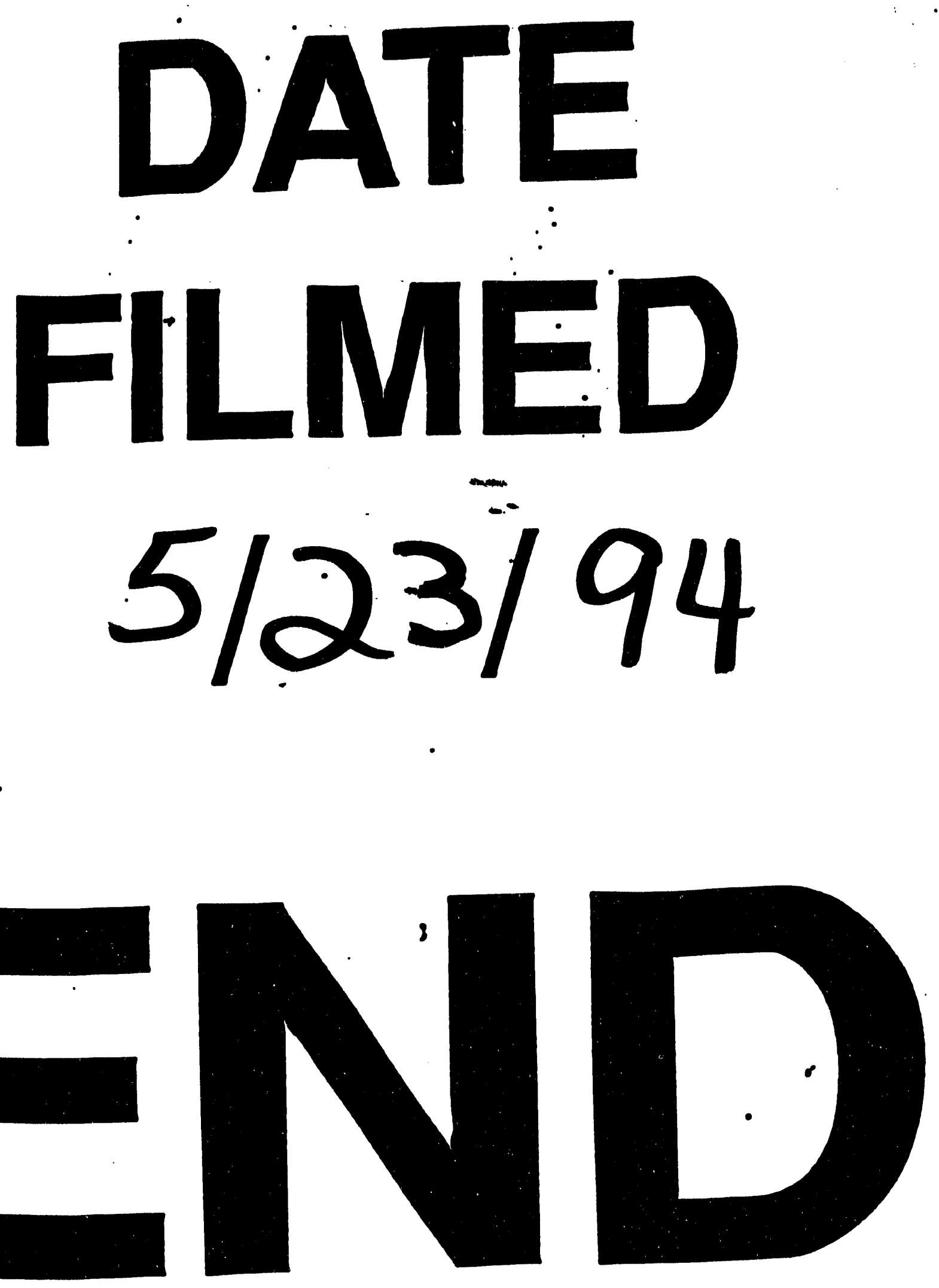


